\title{
Palladium-Catalyzed Dehydrative Cross-Coupling of Allylic Alcohols and $N$-Heterocycles Promoted by a Bicyclic Bridgehead Phosphoramidite Ligand and an Acid Additive
}

\author{
Kyungjun Kang, Jaewook Kim, Ansoo Lee, Woo Youn Kim, and Hyunwoo Kim* \\ Department of Chemistry, KAIST, Daejeon, Korea 34141 \\ Center for Nanomaterials and Chemical Reactions, IBS, Daejeon, Korea 34141 \\ *E-mail: hwkim@kaist.edu
}

\section{General information}

All solvents and commercially available reagents were used without further purification or drying. All reactions were performed in an oven-dried sealed v-vial $(5 \mathrm{~mL}) .{ }^{1} \mathrm{H}$ NMR spectra were recorded on an Agilent Technologies spectrometer DD2 (600 MHz for $\left.{ }^{1} \mathrm{H}\right)$ and reported in ppm, relative to residual protonated solvent peak used as an internal reference. Data for ${ }^{1} \mathrm{H}$ NMR are reported as follows: $\mathrm{s}$ (singlet), d (doublet), t (triplet), q (quartet), dd (doublet of doublet), td (triplet of doublet), m (multiplet). Coupling constants, $J$, were reported in hertz unit $(\mathrm{Hz}) \cdot{ }^{13} \mathrm{C}$ NMR spectra were recorded on an Agilent Technologies spectrometer DD2 (150 MHz for $\left.{ }^{13} \mathrm{C}\right)$ and was fully decoupled by broad band proton decoupling. Chemical shifts were reported in ppm referenced to the center of a triplet at $77.0 \mathrm{ppm}$ of chloroform-d. High-resolution mass spectra (HRMS) were obtained on a Bruker Daltonik micro TOFQII spectrometer in the KAIST Analyst Center. 
2. Optimization of $\mathrm{Pd} /$ briphos L3-catalyzed dehydrative coupling between allyl alchol and benzoic acid

(a)

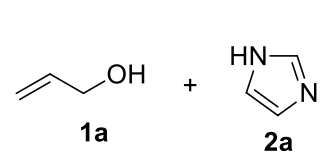

(b)

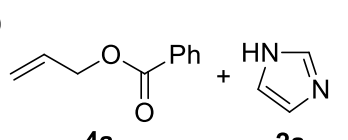

$1 \mathrm{~mol} \% \mathrm{Pd}(\mathrm{dba})_{2}$

$2 \mathrm{~mol} \%$ L3

1.0 equiv Additive

$\mathrm{CH}_{2} \mathrm{Cl}_{2}$

$30{ }^{\circ} \mathrm{C} 12 \mathrm{~h}$
Additive: Yield

(a) None: $0 \%$

(b) $\mathrm{PhCO}_{2} \mathrm{H}$ (5a): $96 \%$

$1 \mathrm{~mol} \% \mathrm{Pd}(\mathrm{dba})_{2}$

$\underset{\mathrm{CH}_{2} \mathrm{Cl}_{2}}{2 \mathrm{~mol} \% \mathrm{L3}} \longrightarrow$

$30^{\circ} \mathrm{C} 12 \mathrm{~h}$

Scheme S1. Pd-catalyzed allylation of imidazole 2a with (a) allyl alcohol 1a or (b) allyl benzoate $4 \mathbf{a}$

Table S1. Pd/L3-catalyzed coupling of allyl alcohol and benzoic acid with various palladium precursors.

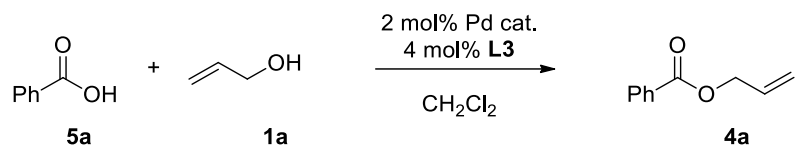

\begin{tabular}{cccccc}
\hline Entry & Pd catalyst & mol\% & Temp $\left({ }^{\circ} \mathrm{C}\right)$ & $\mathrm{T}(\mathrm{h})$ & Yield $(\%)$ \\
\hline 1 & $\mathrm{PdCl}_{2}$ & 2 & 65 & 12 & $\mathrm{n} . \mathrm{r}$ \\
2 & $\mathrm{Pd}(\mathrm{OAc})_{2}$ & 2 & 65 & 12 & 49 \\
3 & $\mathrm{Bis}($ benzonitrile)Pd(II) & 2 & 65 & 12 & $\mathrm{n} . \mathrm{r}$ \\
4 & $\mathrm{Pd}(\mathrm{II})$ acetoacetate & 2 & 65 & 12 & 49 \\
5 & $\mathrm{Pd}(\mathrm{OTf})_{2}$ & 2 & 65 & 12 & 53 \\
6 & $\mathrm{Pd}(\mathrm{OPiv})_{2}$ & 2 & 65 & 12 & 54 \\
7 & $\mathrm{Pd}\left(\mathrm{PPh}_{3}\right)_{4}$ & 2 & 65 & 12 & 44 \\
8 & $\mathrm{Pd}(\mathrm{dba})_{2}$ & 2 & 65 & 12 & 99 \\
\hline
\end{tabular}


Table S2. Solvent Effects on Pd/L3-catalyzed coupling of allyl alcohol and benzoic acid.

\begin{tabular}{|c|c|c|c|c|}
\hline Entry & Solvent & Temp. $\left({ }^{\circ} \mathrm{C}\right)$ & $\mathrm{T}(\mathrm{h})$ & Yield(\%) \\
\hline 1 & 1,4-Dioxane & 30 & 12 & 16 \\
\hline 2 & THF & 30 & 12 & 27 \\
\hline 3 & $\mathrm{CHCl}_{3}$ & 30 & 12 & 62 \\
\hline 4 & DMF & 30 & 12 & 11 \\
\hline 5 & DMSO & 30 & 12 & 17 \\
\hline 6 & $\mathrm{CH}_{2} \mathrm{Cl}_{2}$ & 30 & 12 & 87 \\
\hline 7 & Toluene & 30 & 12 & 56 \\
\hline 8 & $\mathrm{C}_{6} \mathrm{H}_{6}$ & 30 & 12 & 53 \\
\hline 9 & DCE & 30 & 12 & 47 \\
\hline 10 & $\mathrm{MeCN}$ & 30 & 12 & 26 \\
\hline 11 & $\mathrm{CH}_{2} \mathrm{Cl}_{2}$ & 30 & 6 & 86 \\
\hline $12^{\text {[a] }}$ & $\mathrm{CH}_{2} \mathrm{Cl}_{2}$ & 30 & 3 & 99 \\
\hline
\end{tabular}

[a] Conditions: $\mathrm{Pd}(\mathrm{dba})_{2} 2 \mathrm{~mol} \%, \mathbf{L 3} 4 \mathrm{~mol} \%$

\section{Kinetic Experiments}

Initial rate method for the formation of allyl benzoate 4a from allyl alcohol 1a and benzoic acid 5a: Benzoic acid (14.3 mg, $0.1175 \mathrm{mmol}, 1.0$ equiv), allyl alcohol (2-propenol, $8 \mu \mathrm{L}, 0.1175 \mathrm{mmol}, 1$ equiv), L3 (16.2 mg, $0.047 \mathrm{mmol}, 40 \mathrm{~mol} \%$ ), and bis(dibenzylideneacetone)palladium (13.5 mg, 0.0235 mmol, $20 \mathrm{~mol} \%)$ were dissolved in dichloromethane $(0.5 \mathrm{~mL}, 0.235 \mathrm{M})$ in a NMR tube at $\mathrm{N}_{2}$ atmosphere. The conversion of the reaction was determined by ${ }^{1} \mathrm{H}$ NMR spectroscopy at $30{ }^{\circ} \mathrm{C}$. The initial rate method was employed for the determination of the kinetic dependence of reactant concentration. It was obtained from a linear slop for the formation of allyl imidazole under 50\% conversion.

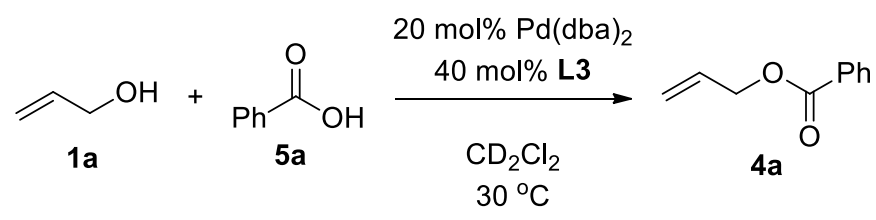

Reaction rate $=0.006[\mathbf{5 a}][\mathbf{1 a}][\mathrm{Pd}]$ 


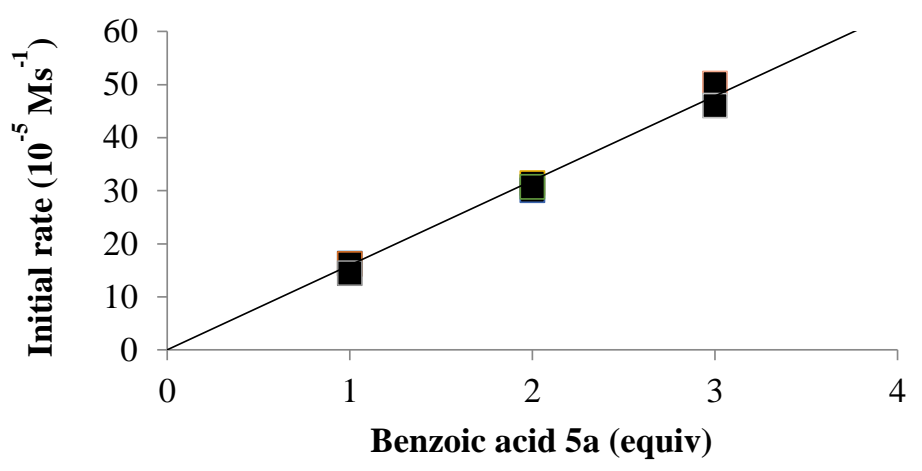

\begin{tabular}{ccccc}
\hline entry & Benzoic acid $(\mathbf{M})$ & Allyl alcohol $(\mathbf{M})$ & $\mathbf{P d}(\mathbf{d b a})_{2}(\mathbf{L 3})_{\mathbf{2}}(\mathbf{M})$ & $\mathbf{k}_{\text {obs }}\left(\mathbf{1 0}^{\mathbf{- 6}} \mathbf{M} / \mathbf{s}\right)$ \\
\hline 1 & 0.235 & 0.235 & 0.047 & 16.22 \\
2 & 0.235 & 0.235 & 0.047 & 16.16 \\
3 & 0.235 & 0.235 & 0.047 & 14.46 \\
4 & 0.470 & 0.235 & 0.047 & 31.40 \\
5 & 0.470 & 0.235 & 0.047 & 30.04 \\
6 & 0.470 & 0.235 & 0.047 & 30.70 \\
7 & 0.940 & 0.235 & 0.047 & 50.07 \\
8 & 0.940 & 0.235 & 0.047 & 50.15 \\
9 & 0.940 & 0.235 & 0.047 & 46.00 \\
\hline
\end{tabular}

Figure S1. Initial rate of allylation of benzoic acid with benzoic acid concentration between $0.235 \mathrm{M}$ and $0.940 \mathrm{M}$.

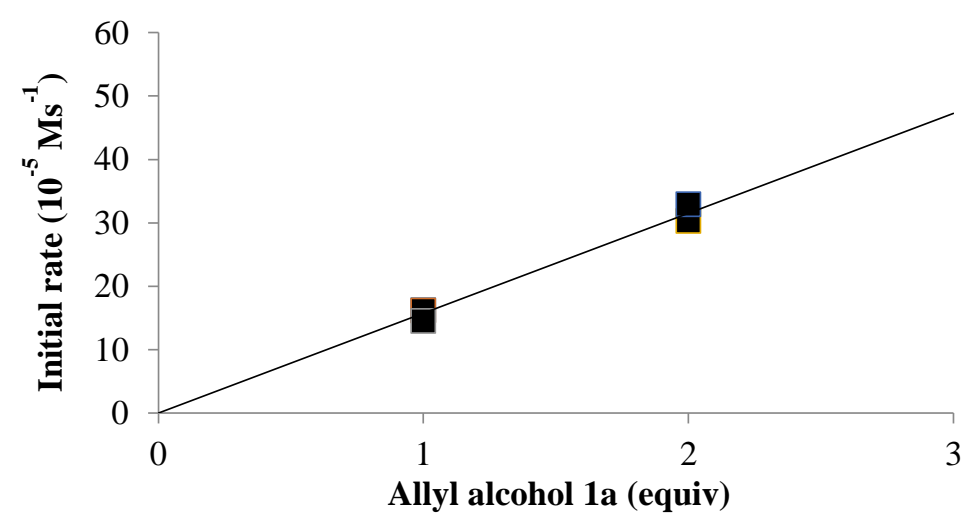

\begin{tabular}{ccccc}
\hline entry & Benzoic acid $(\mathbf{M})$ & Allyl alcohol $(\mathbf{M})$ & $\mathbf{P d}(\mathbf{d b a})_{2}(\mathbf{L 3})_{\mathbf{2}}(\mathbf{M})$ & $\mathbf{k}_{\mathbf{\text { obs }}}\left(\mathbf{1 0}^{\mathbf{6}} \mathbf{\mathbf { M }} / \mathbf{s}\right)$ \\
\hline 1 & 0.235 & 0.235 & 0.047 & 16.22 \\
2 & 0.235 & 0.235 & 0.047 & 16.16 \\
3 & 0.235 & 0.235 & 0.047 & 14.46 \\
4 & 0.235 & 0.470 & 0.047 & 30.29 \\
5 & 0.235 & 0.470 & 0.047 & 32.91 \\
\hline
\end{tabular}

Figure S2. Initial rate of allylation of benzoic acid with allyl alcohol concentration between $0.235 \mathrm{M}$ and $0.470 \mathrm{M}$. 


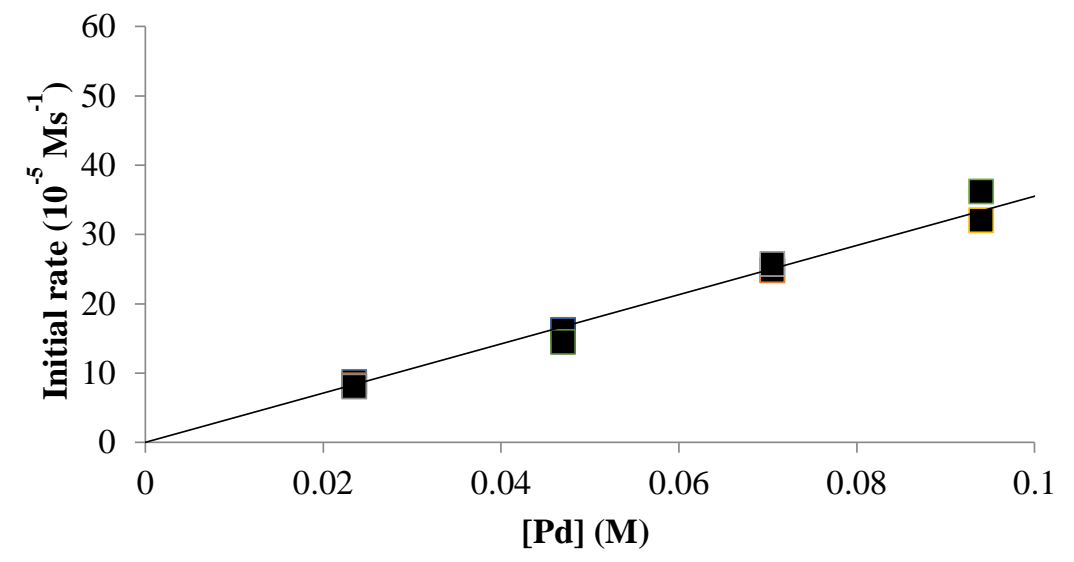

\begin{tabular}{ccccc}
\hline entry & Benzoic acid $(\mathbf{M})$ & Allyl alcohol $(\mathbf{M})$ & $\mathbf{P d}(\mathbf{d b a})_{\mathbf{2}}(\mathbf{L} \mathbf{L})_{\mathbf{2}}(\mathbf{M})$ & $\mathbf{k}_{\mathbf{\text { obs }}}\left(\mathbf{1 0} \mathbf{1 0}^{\mathbf{- 6}} \mathbf{M} / \mathbf{s}\right)$ \\
\hline 1 & 0.235 & 0.235 & 0.0235 & 8.70 \\
2 & 0.235 & 0.235 & 0.0235 & 8.22 \\
3 & 0.235 & 0.235 & 0.0235 & 8.05 \\
4 & 0.235 & 0.235 & 0.047 & 16.22 \\
5 & 0.235 & 0.235 & 0.047 & 16.16 \\
6 & 0.235 & 0.235 & 0.047 & 14.46 \\
7 & 0.235 & 0.235 & 0.0705 & 24.80 \\
8 & 0.235 & 0.235 & 0.0705 & 24.83 \\
9 & 0.235 & 0.235 & 0.0705 & 25.69 \\
10 & 0.235 & 0.235 & 0.0940 & 32.03 \\
11 & 0.235 & 0.235 & 0.0940 & 36.19 \\
\hline
\end{tabular}

Figure S3. Initial rate of allylation of benzoic acid with palladium concentration between $0.0235 \mathrm{M}$ and $0.0940 \mathrm{M}$.

Initial rate method for the formation of allyl imidazole 3a from allyl alcohol 1a and imidazole 2a in the presence of benzoic acid 5a: Imidazole $(8 \mathrm{mg}, 0.1175 \mathrm{mmol}, 1.0$ equiv), allyl alcohol (2propenol, $8 \mu \mathrm{L}, 0.1175 \mathrm{mmol}, 1.0$ equiv), $\mathbf{L 3}(16.2 \mathrm{mg}, 0.047 \mathrm{mmol}, 40 \mathrm{~mol} \%)$, benzoic acid $(0.1175$ mmol, 1.0 equiv) and bis(dibenzylideneacetone)palladium (13.5 mg, $0.0235 \mathrm{mmol}, 20 \mathrm{~mol} \%$ ) were dissolved in dichloromethane $(0.5 \mathrm{~mL}, 0.235 \mathrm{M})$ in a NMR tube at $\mathrm{N}_{2}$ atmosphere. The conversion of the reaction was determined by ${ }^{1} \mathrm{H}$ NMR spectroscopy at $30{ }^{\circ} \mathrm{C}$. The initial rate method was employed for the determination of the kinetic dependence of reactant concentration. It was obtained from a linear slop for the formation of allyl imidazole under $50 \%$ conversion.

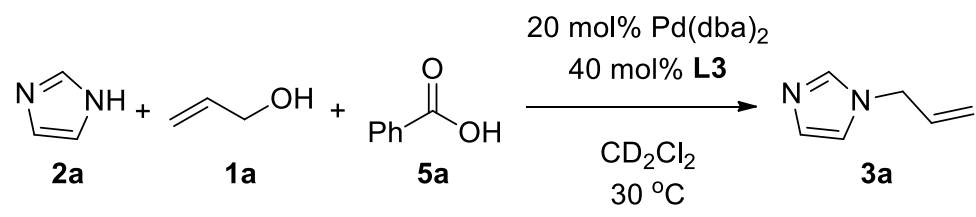

Reaction rate $=0.0033[\mathbf{1 a}][\mathbf{2 a}]^{0}[\mathbf{5 a}][\mathbf{P d}]$ 


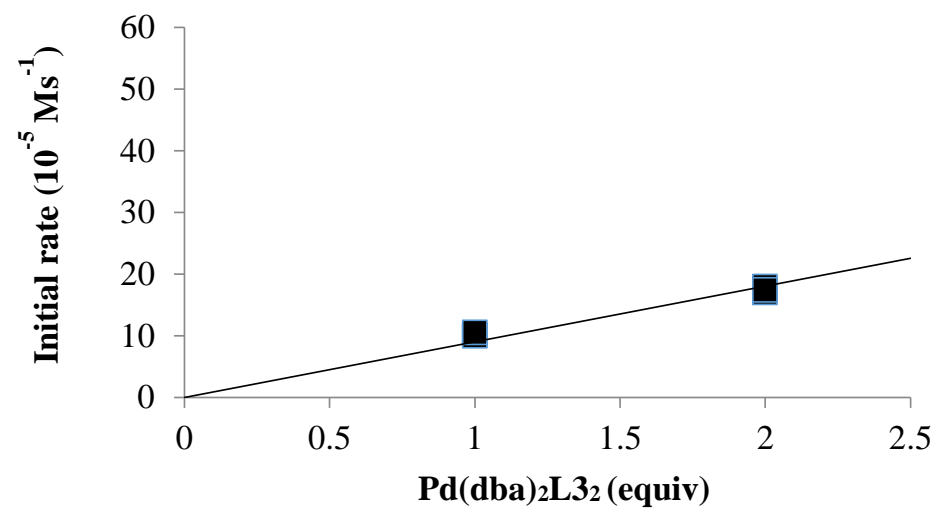

\begin{tabular}{cccccc}
\hline entry & $\begin{array}{c}\text { Imidazole } \\
(\mathbf{M})\end{array}$ & $\begin{array}{c}\text { Allyl alcohol } \\
(\mathbf{M})\end{array}$ & $\begin{array}{c}\text { Benzoic acid } \\
(\mathbf{M})\end{array}$ & $\begin{array}{c}\text { Pd(dba })_{\mathbf{2}}(\mathbf{L} \mathbf{L})_{\mathbf{2}} \\
(\mathbf{M})\end{array}$ & $\begin{array}{c}\mathbf{k}_{\text {obs }}\left(\mathbf{1 0}^{-\mathbf{6}}\right. \\
\mathbf{M} / \mathbf{s})\end{array}$ \\
\hline 1 & 0.235 & 0.235 & 0.235 & 0.0235 & 7.16 \\
2 & 0.235 & 0.235 & 0.235 & 0.0235 & 6.22 \\
3 & 0.235 & 0.235 & 0.235 & 0.0235 & 6.69 \\
4 & 0.235 & 0.235 & 0.235 & 0.047 & 10.10 \\
5 & 0.235 & 0.235 & 0.235 & 0.047 & 10.01 \\
6 & 0.235 & 0.235 & 0.235 & 0.047 & 10.53 \\
7 & 0.235 & 0.235 & 0.235 & 0.0705 & 18.34 \\
8 & 0.235 & 0.235 & 0.235 & 0.0705 & 18.45 \\
9 & 0.235 & 0.235 & 0.235 & 0.0705 & 18.71 \\
10 & 0.235 & 0.235 & 0.235 & 0.094 & 24.52 \\
11 & 0.235 & 0.235 & 0.235 & 0.094 & 25.24 \\
12 & 0.235 & 0.235 & 0.235 & 0.094 & 24.23 \\
\hline
\end{tabular}

Figure S4. Initial rate of allylation of imidazole with palladium concentration between $0.0235 \mathrm{M}$ and $0.094 \mathrm{M}$.

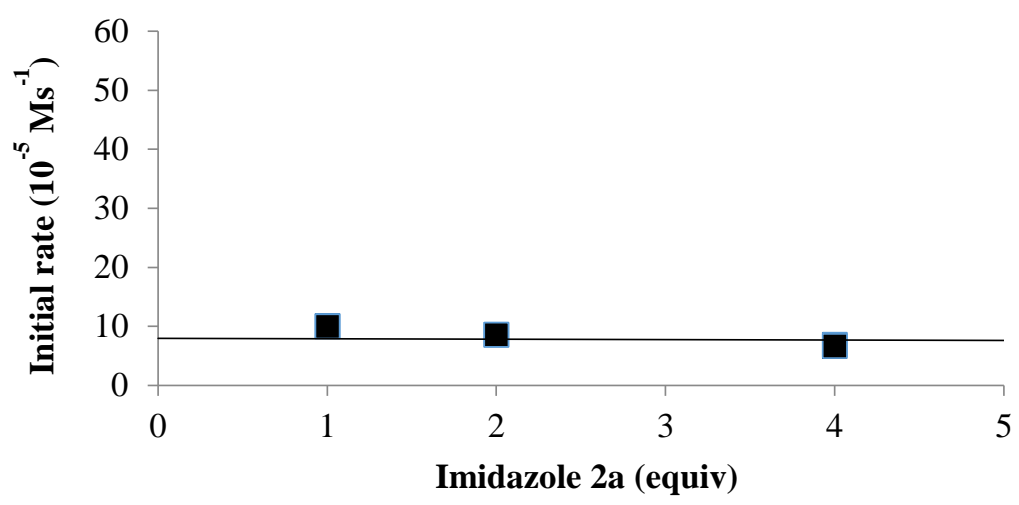

\begin{tabular}{cccccc}
\hline entry & $\begin{array}{c}\text { Imidazole } \\
(\mathbf{M})\end{array}$ & $\begin{array}{c}\text { Allyl alcohol } \\
(\mathbf{M})\end{array}$ & $\begin{array}{c}\text { Benzoic acid } \\
(\mathbf{M})\end{array}$ & $\begin{array}{c}\mathbf{P d}(\mathbf{d b a})_{\mathbf{2}}(\mathbf{L} \mathbf{3})_{\mathbf{2}} \\
(\mathbf{M})\end{array}$ & $\begin{array}{c}\mathbf{k}_{\text {obs }}\left(\mathbf{1 0}^{-\mathbf{6}}\right. \\
\mathbf{M} / \mathbf{s})\end{array}$ \\
\hline 1 & 0.235 & 0.235 & 0.235 & 0.047 & 10.10 \\
2 & 0.235 & 0.235 & 0.235 & 0.047 & 10.01 \\
3 & 0.47 & 0.235 & 0.235 & 0.047 & 8.59 \\
4 & 0.47 & 0.235 & 0.235 & 0.047 & 8.58 \\
5 & 0.47 & 0.235 & 0.235 & 0.047 & 8.01 \\
6 & 0.940 & 0.235 & 0.235 & 0.047 & 6.92 \\
7 & 0.940 & 0.235 & 0.235 & 0.047 & 6.72 \\
\hline
\end{tabular}

Figure S5. Initial rate of allylation of imidazole with imidazole concentration between $0.235 \mathrm{M}$ and $0.940 \mathrm{M}$. 


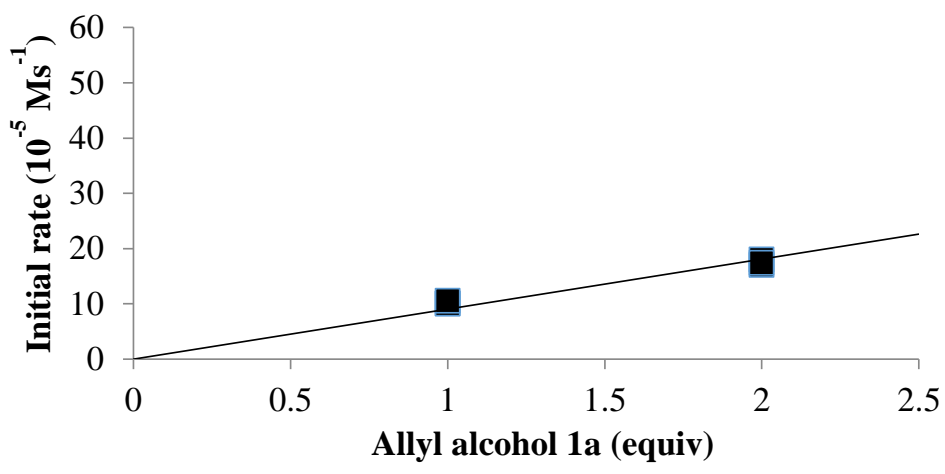

\begin{tabular}{cccccc}
\hline entry & $\begin{array}{c}\text { Imidazole } \\
(\mathbf{M})\end{array}$ & $\begin{array}{c}\text { Allyl alcohol } \\
(\mathbf{M})\end{array}$ & $\begin{array}{c}\text { Benzoic acid } \\
(\mathbf{M})\end{array}$ & $\begin{array}{c}\mathbf{P d}(\mathbf{d b a})_{2}(\mathbf{L 3})_{\mathbf{2}} \\
(\mathbf{M})\end{array}$ & $\begin{array}{c}\mathbf{k}_{\text {obs }}\left(\mathbf{1 0} \mathbf{1 0}^{-\mathbf{6}}\right. \\
\mathbf{M} / \mathbf{s})\end{array}$ \\
\hline 1 & 0.235 & 0.235 & 0.235 & 0.047 & 10.10 \\
2 & 0.235 & 0.235 & 0.235 & 0.047 & 10.01 \\
3 & 0.235 & 0.235 & 0.235 & 0.047 & 10.53 \\
4 & 0.235 & 0.47 & 0.235 & 0.047 & 17.00 \\
5 & 0.235 & 0.47 & 0.235 & 0.047 & 18.00 \\
6 & 0.235 & 0.47 & 0.235 & 0.047 & 17.41 \\
\hline
\end{tabular}

Figure S6. Initial rate of allylation of imidazole with allyl alcohol concentration between $0.235 \mathrm{M}$ and $0.470 \mathrm{M}$.

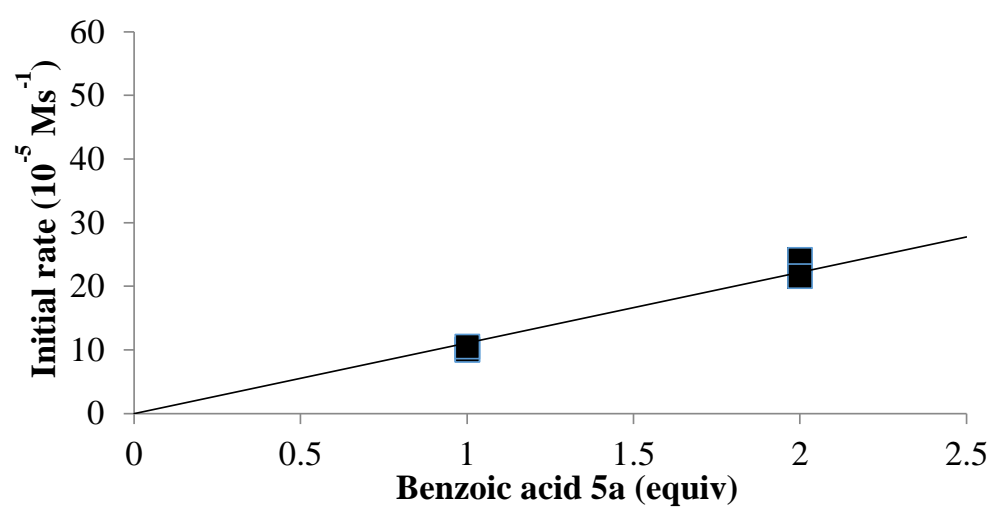

\begin{tabular}{cccccc}
\hline entry & $\begin{array}{c}\text { Imidazole } \\
(\mathbf{M})\end{array}$ & $\begin{array}{c}\text { Allyl alcohol } \\
(\mathbf{M})\end{array}$ & $\begin{array}{c}\text { Benzoic acid } \\
(\mathbf{M})\end{array}$ & $\begin{array}{c}\mathbf{P d}(\mathbf{d b a})_{2}(\mathbf{L 3})_{\mathbf{2}} \\
(\mathbf{M})\end{array}$ & $\begin{array}{c}\mathbf{k}_{\text {obs }}\left(\mathbf{1 0}^{-\mathbf{6}}\right. \\
\mathbf{M} / \mathbf{s})\end{array}$ \\
\hline 1 & 0.235 & 0.235 & 0.235 & 0.047 & 10.10 \\
2 & 0.235 & 0.235 & 0.235 & 0.047 & 10.01 \\
3 & 0.235 & 0.235 & 0.235 & 0.047 & 10.53 \\
4 & 0.235 & 0.235 & 0.47 & 0.047 & 21.62 \\
5 & 0.235 & 0.235 & 0.47 & 0.047 & 24.12 \\
\hline
\end{tabular}

Figure S7. Initial rate of allylation of imidazole with benzoic acid concentration between $0.235 \mathrm{M}$ and $0.470 \mathrm{M}$. 


\section{Comparision between dehydrative coupling and base-mediated alkylation}
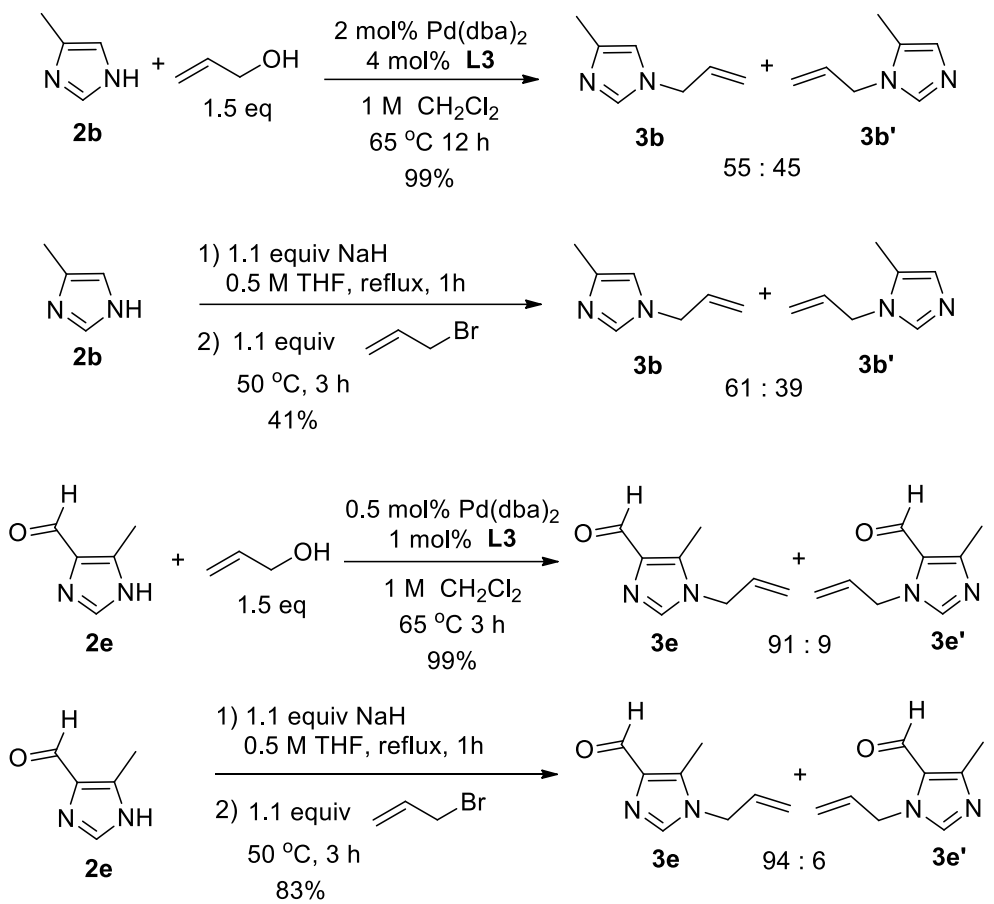

Scheme S2. Comparision between dehydrative coupling and base-mediated alkylation

\section{Experimental procedures}

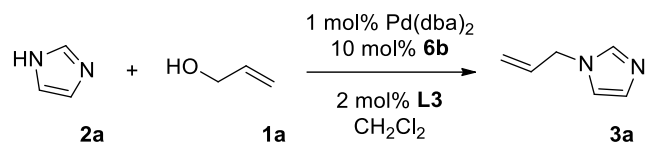

Imidazole (48 mg, $0.7 \mathrm{mmol}, 1.0$ equiv), allyl alcohol (2-propenol, $72 \mu \mathrm{L}, 1.1 \mathrm{mmol}, 1.5$ equiv), L3 (4.9 mg, $0.014 \mathrm{mmol}, 2 \mathrm{~mol} \%$ ), pentafluorophenol (13 $\mu \mathrm{L}, 0.07 \mathrm{mmol}, 0.1$ equiv) and bis(dibenzylideneacetone)palladium(0) (4 $\mathrm{mg}, 0.007 \mathrm{mmol}, 1 \mathrm{~mol} \%)$ were dissolved in dichloromethane $(0.7 \mathrm{~mL}, 1 \mathrm{M})$ in a $5 \mathrm{~mL}$ sealed v-vial at $\mathrm{N}_{2}$ atmosphere. After stirring for $6 \mathrm{~h}$ at $30^{\circ} \mathrm{C}$, volatiles were removed on a rotary evaporator. Purification by flash chromatography on silica gel with dichloromethane/methanol (10:1) afforded product, 3a (73 $\mu \mathrm{L}, 96 \%)$ as a colorless oil. The measured NMR spectra agreed with the literature values. ${ }^{[\mathrm{S} 1]}{ }^{1} \mathrm{H}$ NMR $\left(600 \mathrm{MHz}, \mathrm{CDCl}_{3}\right): \delta 7.47(\mathrm{~s}, 1 \mathrm{H}), 7.06$ (s, $1 \mathrm{H}), 6.89(\mathrm{~s}, 1 \mathrm{H}), 5.98-5.91(\mathrm{~m}, 1 \mathrm{H}), 5.27(\mathrm{~d}, J=10.2 \mathrm{~Hz}, 1 \mathrm{H}), 5.18(\mathrm{~d}, J=16.8 \mathrm{~Hz}, 1 \mathrm{H}), 4.54(\mathrm{~d}, J=$ $6 \mathrm{~Hz}, 2 \mathrm{H}) .{ }^{13} \mathrm{C}$ NMR $\left(150 \mathrm{MHz}, \mathrm{CDCl}_{3}\right): \delta 137.0,132.7,129.4,119.0,118.5,49.3$.

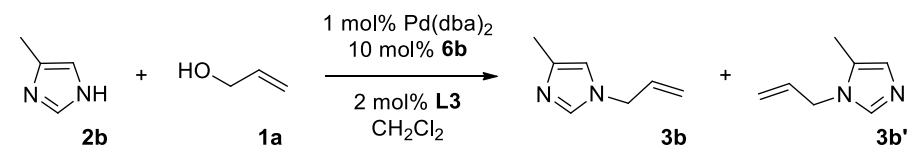

4(5)-Methylimidazole (58 mg, $0.7 \mathrm{mmol}, 1$ equiv), allyl alcohol (2-propenol, $72 \mu \mathrm{L}, 1.1 \mathrm{mmol}, 1.5$ equiv), L3 (4.9 mg, $0.014 \mathrm{mmol}, 2 \mathrm{~mol} \%$ ), pentafluorophenol ( $13 \mu \mathrm{L}, 0.07 \mathrm{mmol}, 0.1$ equiv) and bis(dibenzylideneacetone)palladium $(0) \quad(4 \mathrm{mg}, 0.007 \mathrm{mmol}, 1 \mathrm{~mol} \%)$ were dissolved in dichloromethane $(0.7 \mathrm{~mL}, 1 \mathrm{M})$ in a $5 \mathrm{~mL}$ sealed v-vial under $\mathrm{N}_{2}$ atmosphere. After stirring for $24 \mathrm{~h}$ at $30{ }^{\circ} \mathrm{C}$, volatiles were removed on a rotary evaporator. Purification by flash chromatography on silica 
gel with dichloromethane/methanol (10:1) afforded a mixture of products containing $\mathbf{3 b}$ and $\mathbf{3} \mathbf{b}^{\mathbf{\prime}}$ in a 55:45 ratio (by $\left.\mathrm{H}^{1} \mathrm{NMR}\right)(73 \mu \mathrm{L}, 96 \%)$ as a colorless oil. The measured NMR spectra agreed with the literature values. ${ }^{[\mathrm{S} 5]}$

3b (1-allyl-4-methylimidazole): ${ }^{1} \mathrm{H}$ NMR $\left(600 \mathrm{MHz}, \mathrm{CDCl}_{3}\right): \delta 7.34(\mathrm{~s}, 1 \mathrm{H}), 6.59(\mathrm{~s}, 1 \mathrm{H}), 5.95-5.87$ $(\mathrm{m}, 1 \mathrm{H}), 5.24(\mathrm{~d}, J=10.8 \mathrm{~Hz}, 1 \mathrm{H}), 5.18(\mathrm{~d}, J=17.4 \mathrm{~Hz}, 1 \mathrm{H}), 4.45(\mathrm{~d}, J=6 \mathrm{~Hz}, 2 \mathrm{H}), 2.21(\mathrm{~s}, 3 \mathrm{H}) .{ }^{13} \mathrm{C}$ NMR (150 MHz, $\left.\mathrm{CDCl}_{3}\right): \delta 138.6,136.3,133.2,118.4,115.6,49.4,13.8$.

3b' (1-allyl-5-methylimidazole): ${ }^{1} \mathrm{H}$ NMR $\left(600 \mathrm{MHz}, \mathrm{CDCl}_{3}\right): \delta 7.37$ (s, 1H), $6.76(\mathrm{~s}, 1 \mathrm{H}), 5.95-5.87$ $(\mathrm{m}, 1 \mathrm{H}), 5.22(\mathrm{~d}, J=10.8 \mathrm{~Hz}, 1 \mathrm{H}), 4.98(\mathrm{~d}, J=16.8 \mathrm{~Hz}, 1 \mathrm{H}), 4.44(\mathrm{~d}, J=6 \mathrm{~Hz}, 2 \mathrm{H}), 2.15(\mathrm{~s}, 3 \mathrm{H}) .{ }^{13} \mathrm{C}$ $\operatorname{NMR}\left(150 \mathrm{MHz}, \mathrm{CDCl}_{3}\right): \delta 137.0,133.0,127.4,127.1,117.6,47.0,9.2$.

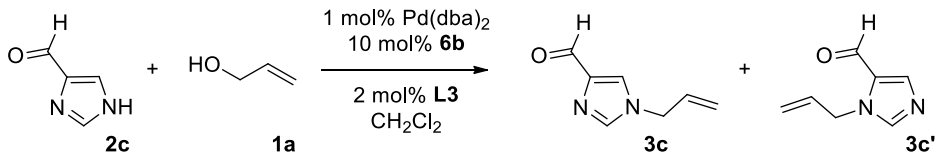

4-Imidazolecarboxyaldehyde ( $68 \mathrm{mg}, 0.7 \mathrm{mmol}, 1$ equiv), allyl alcohol (2-propenol, $72 \mu \mathrm{L}, 1.1 \mathrm{mmol}$, 1.5 equiv), L3 (4.9 mg, $0.014 \mathrm{mmol}, 2 \mathrm{~mol} \%)$, pentafluorophenol (13 $\mu \mathrm{L}, 0.07 \mathrm{mmol}, 0.1$ equiv) and bis(dibenzylideneacetone)palladium(0) (4 $\mathrm{mg}, 0.007 \mathrm{mmol}, 1 \mathrm{~mol} \%)$ were dissolved in dichloromethane $(0.7 \mathrm{~mL}, 1 \mathrm{M})$ in a $5 \mathrm{~mL}$ sealed v-vial under $\mathrm{N}_{2}$ atmosphere. After stirring for $6 \mathrm{~h}$ at $30{ }^{\circ} \mathrm{C}$, volatiles were removed on a rotary evaporator. Purification by flash chromatography on silica gel with dichloromethane/methanol (10:1) afforded a mixture of products containing $\mathbf{3 c}$ and $\mathbf{3} \mathbf{c}^{\prime}$ in a 92:8 ratio (by $\left.\mathrm{H}^{1} \mathrm{NMR}\right)(92 \mu \mathrm{L}, 97 \%$ ) as a colorless oil.

3c (1-allyl-4-imidazolecarboxyaldehyde): ${ }^{1} \mathrm{H}$ NMR (600 MHz, $\left.\mathrm{CDCl}_{3}\right): \delta 9.82(\mathrm{~s}, 1 \mathrm{H}), 7.60(\mathrm{~s}, 1 \mathrm{H})$, $7.53(\mathrm{~s}, 1 \mathrm{H}), 5.96-5.90(\mathrm{~m}, 1 \mathrm{H}), 5.33(\mathrm{~d}, J=10.2 \mathrm{~Hz}, 1 \mathrm{H}), 5.22(\mathrm{~d}, J=16.8 \mathrm{~Hz}, 1 \mathrm{H}), 4.59(\mathrm{~d}, J=5.4$ $\mathrm{Hz}, 2 \mathrm{H}) .{ }^{13} \mathrm{C} \mathrm{NMR}\left(150 \mathrm{MHz}, \mathrm{CDCl}_{3}\right): \delta 185.9,142.3,138.5,131.3,124.4,119.8,49.8$.

3c' (1-allyl-5-imidazolecarboxyaldehyde): ${ }^{1} \mathrm{H}$ NMR (600 MHz, $\left.\mathrm{CDCl}_{3}\right): \delta 9.71(\mathrm{~s}, 1 \mathrm{H}), 7.77(\mathrm{~s}, 1 \mathrm{H})$, $7.65(\mathrm{~s}, 1 \mathrm{H}), 5.96-5.90(\mathrm{~m}, 1 \mathrm{H}), 5.22(\mathrm{~d}, J=10.2 \mathrm{~Hz}, 1 \mathrm{H}), 5.08(\mathrm{~d}, J=16.8 \mathrm{~Hz}, 1 \mathrm{H}), 4.90(\mathrm{~d}, J=5.4$ $\mathrm{Hz}, 2 \mathrm{H}) .{ }^{13} \mathrm{C} \mathrm{NMR}\left(150 \mathrm{MHz}, \mathrm{CDCl}_{3}\right): \delta 179.0,143.5,143.3,132.2,118.4,48.9 ; \mathrm{HRMS}$ (EI) $\mathrm{m} / \mathrm{z}$ calcd for $\mathrm{C}_{7} \mathrm{H}_{8} \mathrm{~N}_{2} \mathrm{O}[\mathrm{Na}]^{+}: 159.0534$, found : 159.0533 .

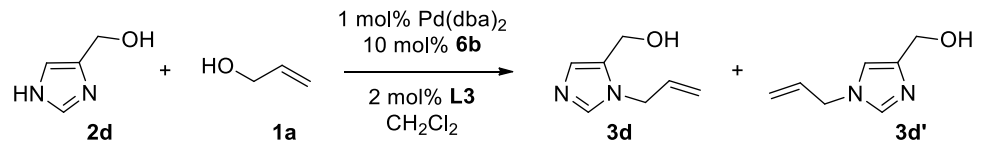

4(5)-(Hydroxymethyl)imidazole (68 mg, $0.7 \mathrm{mmol}, 1$ equiv), allyl alcohol (2-propenol, $72 \mu \mathrm{L}, 1.1$ mmol, 1.5 equiv), L3 (4.9 mg, $0.014 \mathrm{mmol}, 2 \mathrm{~mol} \%)$, pentafluorophenol ( $13 \mu \mathrm{L}, 0.07 \mathrm{mmol}, 0.1$ equiv) and bis(dibenzylideneacetone)palladium(0) $(4 \mathrm{mg}, 0.007 \mathrm{mmol}, 1 \mathrm{~mol} \%$ ) were dissolved in dichloromethane $(0.7 \mathrm{~mL}, 1 \mathrm{M})$ in a $5 \mathrm{~mL}$ sealed v-vial under $\mathrm{N}_{2}$ atmosphere. After stirring for $6 \mathrm{~h}$ at $30{ }^{\circ} \mathrm{C}$, volatiles were removed on a rotary evaporator. Purification by flash chromatography on silica gel with dichloromethane/methanol (10:1) afforded a mixture of products containing $\mathbf{3 d}$ and $\mathbf{3 d}$ ' in a $7: 3$ ratio (by $\left.\mathrm{H}^{1} \mathrm{NMR}\right)(87 \mu \mathrm{L}, 90 \%)$ as a colorless oil.

3d (1-allyl-5-(Hydroxymethyl)imidazole): ${ }^{1} \mathrm{H}$ NMR (600 MHz, $\left.\mathrm{CDCl}_{3}\right): \delta 7.39$ (s, 1H), $6.84(\mathrm{~s}, 1 \mathrm{H})$, $5.95-5.88(\mathrm{~m}, 1 \mathrm{H}), 5.26(\mathrm{~d}, J=10.2 \mathrm{~Hz}, 1 \mathrm{H}), 5.19(\mathrm{~d}, J=16.8 \mathrm{~Hz}, 1 \mathrm{H}), 4.55(\mathrm{~s}, 2 \mathrm{H}), 4.48(\mathrm{~d}, J=6 \mathrm{~Hz}$, $2 \mathrm{H}) .{ }^{13} \mathrm{C}$ NMR $\left(150 \mathrm{MHz}, \mathrm{CDCl}_{3}\right): \delta 138.2,133.1,131.1,128.2,117.7,53.8,47.3$.

3d' (1-allyl-4-(Hydroxymethyl)imidazole): ${ }^{1} \mathrm{H}$ NMR (600 MHz, $\left.\mathrm{CDCl}_{3}\right) \delta 7.41$ (s, 1H), $6.89(\mathrm{~s}, 1 \mathrm{H})$, $5.99-5.90(\mathrm{~m}, 1 \mathrm{H}), 5.22(\mathrm{~d}, J=10.2 \mathrm{~Hz}, 1 \mathrm{H}), 5.06(\mathrm{~d}, J=16.8 \mathrm{~Hz}, 1 \mathrm{H}), 4.63(\mathrm{~d}, J=6 \mathrm{~Hz}, 2 \mathrm{H}), 4.57(\mathrm{~s}$, $2 \mathrm{H}) .{ }^{13} \mathrm{C}$ NMR $\left(150 \mathrm{MHz}, \mathrm{CDCl}_{3}\right): \delta 142.6,136.8,132.5,118.7,116.3,58.1,49.4 ;$ HRMS (EI) $\mathrm{m} / \mathrm{z}$ 
calcd for $\mathrm{C}_{7} \mathrm{H}_{10} \mathrm{~N}_{2} \mathrm{O}[\mathrm{Na}]^{+}$: 161.0691 , found : 161.0689 .

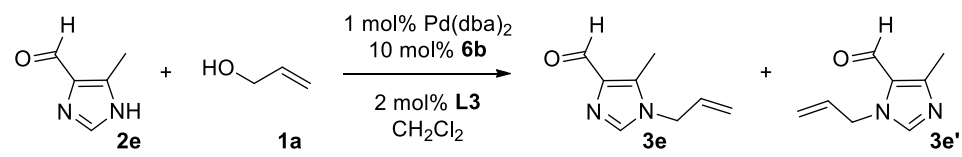

5-Methylimidazole-4-carboxaldehyde ( $79 \mathrm{mg}, 0.7 \mathrm{mmol}, 1$ equiv), allyl alcohol (2-propenol, $72 \mu \mathrm{L}$, $1.1 \mathrm{mmol}, 1.5$ equiv), L3 (4.9 mg, $0.014 \mathrm{mmol}, 2 \mathrm{~mol} \%)$, pentafluorophenol ( $13 \mu \mathrm{L}, 0.07 \mathrm{mmol}, 0.1$ equiv) and bis(dibenzylideneacetone)palladium(0) $(4 \mathrm{mg}, 0.007 \mathrm{mmol}, 1 \mathrm{~mol} \%)$ were dissolved in dichloromethane $(0.7 \mathrm{~mL}, 1 \mathrm{M})$ in a $5 \mathrm{~mL}$ sealed $\mathrm{v}$-vial under $\mathrm{N}_{2}$ atmosphere. After stirring for $6 \mathrm{~h}$ at $30{ }^{\circ} \mathrm{C}$, volatiles were removed on a rotary evaporator. Purification by flash chromatography on silica gel with dichloromethane/methanol (10:1) afforded a mixture of products containing $\mathbf{3 e}$ and $\mathbf{3} \mathbf{e}^{\prime}$ in a 91:9 ratio (by $\left.\mathrm{H}^{1} \mathrm{NMR}\right)(99 \mu \mathrm{L}, 94 \%$ ) as a colorless oil.

3e (1-allyl-4-methylimidazole-5-carboxaldehyde): ${ }^{1} \mathrm{H}$ NMR $\left(600 \mathrm{MHz}, \mathrm{CDCl}_{3}\right): \delta 9.77(\mathrm{~s}, 1 \mathrm{H}), 7.34$ (s, $1 \mathrm{H}), 5.82-5.77(\mathrm{~m}, 1 \mathrm{H}), 5.16(\mathrm{~d}, J=10.2 \mathrm{~Hz}, 1 \mathrm{H}), 4.89(\mathrm{~d}, J=16.8 \mathrm{~Hz}, 1 \mathrm{H}), 4.42(\mathrm{~d}, J=4.8 \mathrm{~Hz}, 2 \mathrm{H})$, $2.35(\mathrm{~s}, 3 \mathrm{H}) .{ }^{13} \mathrm{C} \mathrm{NMR}\left(150 \mathrm{MHz}, \mathrm{CDCl}_{3}\right): \delta 187.3,137.7,137.5,135.3,131.3,118.5,46.7,9.0$.

3e' (1-allyl-5-methylimidazole-4-carboxaldehyde): ${ }^{1} \mathrm{H} \mathrm{NMR}\left(600 \mathrm{MHz}, \mathrm{CDCl}_{3}\right): \delta 9.68(\mathrm{~s}, 1 \mathrm{H}), 7.44$ $(\mathrm{s}, 1 \mathrm{H}), 5.92-5.87(\mathrm{~m}, 1 \mathrm{H}), 5.08(\mathrm{~d}, J=10.2 \mathrm{~Hz}, 1 \mathrm{H}), 4.97(\mathrm{~d}, J=16.8 \mathrm{~Hz}, 1 \mathrm{H}), 4.75(\mathrm{~d}, J=4.8 \mathrm{~Hz}$, $2 \mathrm{H}), 2.35$ (s, 3H). ${ }^{13} \mathrm{C} \mathrm{NMR}\left(150 \mathrm{MHz}, \mathrm{CDCl}_{3}\right): \delta 178.1,152.8,141.5,132.4,125.9,117.8,48.6,12.9$; HRMS (EI) $\mathrm{m} / \mathrm{z}$ calcd for $\mathrm{C}_{8} \mathrm{H}_{10} \mathrm{~N}_{2} \mathrm{O}[\mathrm{Na}]^{+}: 173.0691$, found : 173.0685 .

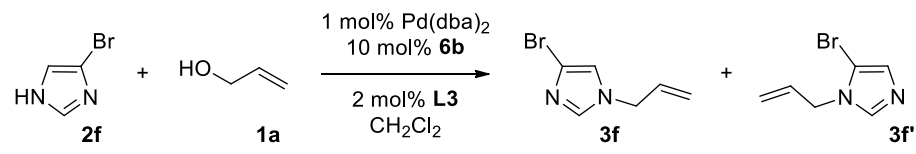

4-Bromo-1H-imidazole (79 mg, $0.7 \mathrm{mmol}, 1$ equiv), allyl alcohol (2-propenol, $72 \mu \mathrm{L}, 1.1 \mathrm{mmol}, 1.5$ equiv), L3 (4.9 mg, $0.014 \mathrm{mmol}, 2 \mathrm{~mol} \%$ ), pentafluorophenol (13 $\mu \mathrm{L}, 0.07 \mathrm{mmol}, 0.1$ equiv) and bis(dibenzylideneacetone)palladium(0) (4 $\mathrm{mg}, 0.007 \mathrm{mmol}, 1 \mathrm{~mol} \%)$ were dissolved in dichloromethane $(0.7 \mathrm{~mL}, 1 \mathrm{M})$ in a $5 \mathrm{~mL}$ sealed $\mathrm{v}$-vial under $\mathrm{N}_{2}$ atmosphere. After stirring for $6 \mathrm{~h}$ at $30{ }^{\circ} \mathrm{C}$, volatiles were removed on a rotary evaporator. Purification by flash chromatography on silica gel with dichloromethane/methanol (10:1) afforded a mixture of products containing $\mathbf{3 f}$ and $\mathbf{3} \mathbf{f}^{\prime}$ in a 77:23 ratio (by $\left.\mathrm{H}^{1} \mathrm{NMR}\right)(122 \mu \mathrm{L}, 93 \%)$ as a colorless oil.

3f (1-allyl-4-bromo-1H-imidazole): ${ }^{1} \mathrm{H} \mathrm{NMR}\left(600 \mathrm{MHz}, \mathrm{CDCl}_{3}\right): \delta 7.55(\mathrm{~s}, 1 \mathrm{H}), 7.04(\mathrm{~s}, 1 \mathrm{H}), 5.96-5.90$ $(\mathrm{m}, 1 \mathrm{H}), 5.30(\mathrm{~d}, J=10.2 \mathrm{~Hz}, 1 \mathrm{H}), 5.11(\mathrm{~d}, J=17.4 \mathrm{~Hz}, 1 \mathrm{H}), 4.55(\mathrm{~d}, J=4.8 \mathrm{~Hz}, 2 \mathrm{H}) .{ }^{13} \mathrm{C}$ NMR $(150$ $\left.\mathrm{MHz}, \mathrm{CDCl}_{3}\right): \delta 137.8,131.8,129.7,118.7,48.1,29.6$.

3f' (1-allyl-5-bromo-1H-imidazole): ${ }^{1} \mathrm{H}$ NMR $\left(600 \mathrm{MHz}, \mathrm{CDCl}_{3}\right): \delta 7.37$ (s, $\left.1 \mathrm{H}\right), 6.88(\mathrm{~s}, 1 \mathrm{H}), 5.96-$ $5.90(\mathrm{~m}, 1 \mathrm{H}), 5.33(\mathrm{~d}, J=10.2 \mathrm{~Hz}, 1 \mathrm{H}), 5.25(\mathrm{~d}, J=16.8 \mathrm{~Hz}, 1 \mathrm{H}), 4.51(\mathrm{~d}, J=4.8 \mathrm{~Hz}, 2 \mathrm{H}) .{ }^{13} \mathrm{C} \mathrm{NMR}$ $\left(150 \mathrm{MHz}, \mathrm{CDCl}_{3}\right): \delta 136.6,131.9,119.4,118.2,49.9,29.2 ; \mathrm{HRMS}$ (EI) $\mathrm{m} / \mathrm{z}$ calcd for $\mathrm{C}_{6} \mathrm{H}_{8} \mathrm{BrN}_{2}[\mathrm{H}]^{+}$: 186.9871 , found : 186.9855 .

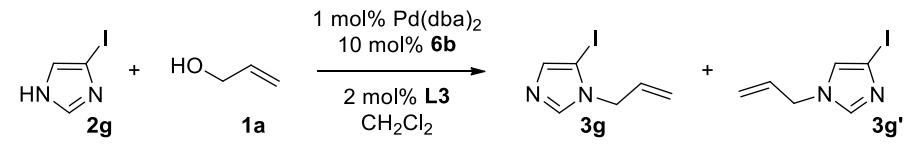

4-Iodo-1H-imidazole (79 mg, $0.7 \mathrm{mmol}, 1$ equiv), allyl alcohol (2-propenol, $72 \mu \mathrm{L}, 1.1 \mathrm{mmol}, 1.5$ equiv), L3 (4.9 mg, $0.014 \mathrm{mmol}, 2 \mathrm{~mol} \%$ ), pentafluorophenol ( $13 \mu \mathrm{L}, 0.07 \mathrm{mmol}, 0.1$ equiv) and bis(dibenzylideneacetone)palladium(0) (4 $\mathrm{mg}, 0.007 \mathrm{mmol}, 1 \mathrm{~mol} \%)$ were dissolved in dichloromethane $(0.7 \mathrm{~mL}, 1 \mathrm{M})$ in a $5 \mathrm{~mL}$ sealed v-vial under $\mathrm{N}_{2}$ atmosphere. After stirring for $24 \mathrm{~h}$ at 
$50_{-}^{\circ} \mathrm{C}$, volatiles were removed on a rotary evaporator. Purification by flash chromatography on silica gel with dichloromethane/methanol (10:1) afforded a mixture of products containing $\mathbf{3 g}$ and $\mathbf{3} \mathbf{g}$ ' in a 55:45 ratio (by $\left.\mathrm{H}^{1} \mathrm{NMR}\right)(128 \mu \mathrm{L}, 78 \%)$ as a colorless oil.

$3 g$ (1-allyl-4-iodo-1H-imidazole): ${ }^{1} \mathrm{H}$ NMR $\left(600 \mathrm{MHz}, \mathrm{CDCl}_{3}\right): \delta 7.64(\mathrm{~s}, 1 \mathrm{H}), 7.15(\mathrm{~s}, 1 \mathrm{H}), 5.95-5.89$ $(\mathrm{m}, 1 \mathrm{H}), 5.30(\mathrm{~d}, J=10.2 \mathrm{~Hz}, 1 \mathrm{H}), 5.08(\mathrm{~d}, J=16.8 \mathrm{~Hz}, 1 \mathrm{H}), 4.54(\mathrm{~d}, J=5.4 \mathrm{~Hz}, 2 \mathrm{H}) .{ }^{13} \mathrm{C}$ NMR $(150$ $\left.\mathrm{MHz}, \mathrm{CDCl}_{3}\right): \delta 139.4,137.0,132.0,118.7,49.9$.

3g' (1-allyl-5-iodo-1H-imidazole): ${ }^{1} \mathrm{H}$ NMR $\left(600 \mathrm{MHz}, \mathrm{CDCl}_{3}\right): \delta 7.61(\mathrm{~s}, 1 \mathrm{H}), 7.11(\mathrm{~s}, 1 \mathrm{H})$, 5.95-5.89 $(\mathrm{m}, 1 \mathrm{H}), 5.30(\mathrm{~d}, J=10.2 \mathrm{~Hz}, 1 \mathrm{H}), 5.08(\mathrm{~d}, J=16.8 \mathrm{~Hz}, 1 \mathrm{H}), 4.58(\mathrm{~d}, J=4.8 \mathrm{~Hz}, 2 \mathrm{H}) .{ }^{13} \mathrm{C}$ NMR $(150$ $\mathrm{MHz}, \mathrm{CDCl}_{3}$ ): $\delta$ 139.2, 137.4, 132.0, 118.7, 49.9; HRMS (EI) m/z calcd for $\mathrm{C}_{6} \mathrm{H}_{8} \mathrm{IN}_{2}[\mathrm{H}]^{+}: 234.9732$, found : 234.9717 .

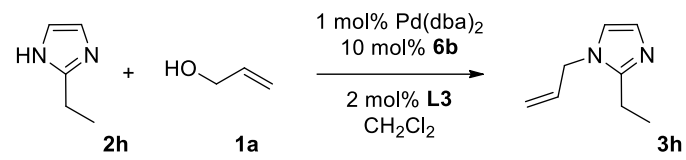

2-Ethylimidazole ( $67 \mathrm{mg}, 0.7 \mathrm{mmol}, 1$ equiv), allyl alcohol (2-propenol, $72 \mu \mathrm{L}, 1.1 \mathrm{mmol}, 1.5$ equiv), L3 (4.9 mg, $0.014 \mathrm{mmol}, 2 \mathrm{~mol} \%)$, pentafluorophenol (13 $\mu \mathrm{L}, 0.07 \mathrm{mmol}, 0.1$ equiv) and bis(dibenzylideneacetone)palladium(0) (4 $\mathrm{mg}, 0.007 \mathrm{mmol}, 1 \mathrm{~mol} \%)$ were dissolved in dichloromethane $(0.7 \mathrm{~mL}, 1 \mathrm{M})$ in a $5 \mathrm{~mL}$ sealed v-vial under $\mathrm{N}_{2}$ atmosphere. After stirring for $24 \mathrm{~h}$ at $30{ }^{\circ} \mathrm{C}$, volatiles were removed on a rotary evaporator. Purification by flash chromatography on silica gel with dichloromethane/methanol (10:1) afforded product, $\mathbf{3 h}(93 \mu \mathrm{L}, 98 \%)$ as a colorless oil. ${ }^{1} \mathrm{H}$ NMR (600 MHz, $\left.\mathrm{CDCl}_{3}\right): \delta 6.95(\mathrm{~s}, 1 \mathrm{H}), 6.80(\mathrm{~s}, 1 \mathrm{H}), 5.93-5.87(\mathrm{~m}, 1 \mathrm{H}), 5.23(\mathrm{~d}, J=10.2 \mathrm{~Hz}, 1 \mathrm{H})$, $5.02(\mathrm{~d}, J=17.4 \mathrm{~Hz}, 1 \mathrm{H}), 4.46(\mathrm{~d}, J=4.8 \mathrm{~Hz}, 2 \mathrm{H}), 2.67(\mathrm{q}, J=7.8 \mathrm{~Hz}, 2 \mathrm{H}), 1.33(\mathrm{t}, J=7.2 \mathrm{~Hz}, 3 \mathrm{H}) .{ }^{13} \mathrm{C}$ NMR $\left(150 \mathrm{MHz}, \mathrm{CDCl}_{3}\right): \delta 149.2,132.9,127.1,119.2,117.4,47.7,19.9,12.0 ; \mathrm{HRMS}$ (EI) m/z calcd for $\mathrm{C}_{8} \mathrm{H}_{12} \mathrm{~N}_{2}[\mathrm{Na}]^{+}:$: 159.0898, found : 159.0891 .

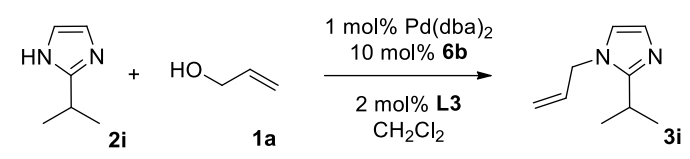

2-Isopropylimidazole (77 mg, $0.7 \mathrm{mmol}, 1$ equiv), allyl alcohol (2-propenol, $72 \mu \mathrm{L}, 1.1 \mathrm{mmol}, 1.5$ equiv), L3 (4.9 mg, $0.014 \mathrm{mmol}, 2 \mathrm{~mol} \%$ ), pentafluorophenol ( $13 \mu \mathrm{L}, 0.07 \mathrm{mmol}, 0.1$ equiv) and bis(dibenzylideneacetone)palladium(0) (4 $\mathrm{mg}, 0.007 \mathrm{mmol}, 1 \mathrm{~mol} \%)$ were dissolved in dichloromethane $(0.7 \mathrm{~mL}, 1 \mathrm{M})$ in a $5 \mathrm{~mL}$ sealed v-vial under $\mathrm{N}_{2}$ atmosphere. After stirring for $24 \mathrm{~h}$ at $50{ }^{\circ} \mathrm{C}$, volatiles were removed on a rotary evaporator. Purification by flash chromatography on silica gel with dichloromethane/methanol (10:1) afforded product, 3i $(102 \mu \mathrm{L}, 97 \%)$ as a colorless oil. ${ }^{1} \mathrm{H}$ NMR (600 MHz, $\left.\mathrm{CDCl}_{3}\right): \delta 6.91(\mathrm{~s}, 1 \mathrm{H}), 6.72(\mathrm{~s}, 1 \mathrm{H}), 5.90-5.84(\mathrm{~m}, 1 \mathrm{H}), 5.18(\mathrm{~d}, J=10.2 \mathrm{~Hz}, 1 \mathrm{H})$, $4.97(\mathrm{~d}, J=17.4 \mathrm{~Hz}, 1 \mathrm{H}), 4.44(\mathrm{~d}, J=4.8 \mathrm{~Hz}, 2 \mathrm{H}), 2.93(\mathrm{~m}, 1 \mathrm{H}), 1.26(\mathrm{~d}, J=7.2 \mathrm{~Hz}, 6 \mathrm{H}) .{ }^{13} \mathrm{C}$ NMR $(150$ $\left.\mathrm{MHz}, \mathrm{CDCl}_{3}\right): \delta 152.7,133.1,126.9,118.8,117.2,47.6,25.7,21.6 ; \mathrm{HRMS}(\mathrm{EI}) \mathrm{m} / \mathrm{z}$ calcd for $\mathrm{C}_{9} \mathrm{H}_{14} \mathrm{~N}_{2}$ $[\mathrm{Na}]^{+}: 173.1055$, found : 173.1040 .

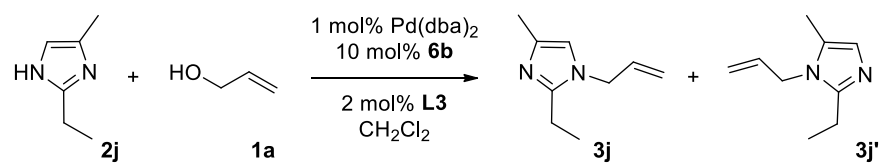

2-Ethyl-4-methylimidazole ( $79 \mathrm{mg}, 0.7 \mathrm{mmol}, 1$ equiv), allyl alcohol (2-propenol, $72 \mu \mathrm{L}, 1.1 \mathrm{mmol}$, 1.5 equiv), L3 (4.9 mg, $0.014 \mathrm{mmol}, 2 \mathrm{~mol} \%)$, pentafluorophenol (13 $\mu \mathrm{L}, 0.07 \mathrm{mmol}, 0.1$ equiv) and 
bis(dibenzylideneacetone)palladium(0) (4 $\mathrm{mg}, 0.007 \mathrm{mmol}, \quad 1 \mathrm{~mol} \%)$ were dissolved in dichloromethane $(0.7 \mathrm{~mL}, 1 \mathrm{M})$ in a $5 \mathrm{~mL}$ sealed v-vial under $\mathrm{N}_{2}$ atmosphere. After stirring for $24 \mathrm{~h}$ at $30{ }^{\circ} \mathrm{C}$, volatiles were removed on a rotary evaporator. Purification by flash chromatography on silica gel with dichloromethane/methanol (10:1) afforded a mixture of products containing $\mathbf{3 j}$ and $\mathbf{3 j}$ ' in a 89:11 ratio (by $\left.\mathrm{H}^{1} \mathrm{NMR}\right)(75 \mu \mathrm{L}, 71 \%)$ as a colorless oil.

3j (1-allyl-2-ethyl-4-methyl-1H-imidazole): ${ }^{1} \mathrm{H}$ NMR (600 MHz, $\left.\mathrm{CDCl}_{3}\right): \delta 6.50$ (s, 1H), 5.92-5.85 (m, $1 \mathrm{H}), 5.21(\mathrm{~d}, J=10.2 \mathrm{~Hz}, 1 \mathrm{H}), 5.04(\mathrm{~d}, J=17.4 \mathrm{~Hz}, 1 \mathrm{H}), 4.39(\mathrm{~d}, J=5.4 \mathrm{~Hz}, 2 \mathrm{H}), 2.64(\mathrm{q}, J=7.8 \mathrm{~Hz}$, 2H), $2.19(\mathrm{~s}, 3 \mathrm{H}), 1.31(\mathrm{t}, J=7.2 \mathrm{~Hz}, 4 \mathrm{H}) .{ }^{13} \mathrm{C} \mathrm{NMR}\left(150 \mathrm{MHz}, \mathrm{CDCl}_{3}\right): \delta 148.6,135.8,133.1,117.3$, $115.5,47.9,20.0,13.4,12.4$.

3j' (1-allyl-2-ethyl-5-methyl-1H-imidazole): ${ }^{1} \mathrm{H}$ NMR (600 MHz, $\left.\mathrm{CDCl}_{3}\right): \delta 6.70(\mathrm{~s}, 1 \mathrm{H}), 5.92-5.85$ (m, $1 \mathrm{H}), 5.17(\mathrm{~d}, J=8.4 \mathrm{~Hz}, 1 \mathrm{H}), 4.80(\mathrm{~d}, J=17.4 \mathrm{~Hz}, 1 \mathrm{H}), 4.39(\mathrm{~d}, J=5.4 \mathrm{~Hz}, 2 \mathrm{H}), 2.64(\mathrm{q}, J=7.8 \mathrm{~Hz}$, $2 \mathrm{H}), 2.13(\mathrm{~s}, 3 \mathrm{H}), 1.31(\mathrm{t}, J=7.2 \mathrm{~Hz}, 3 \mathrm{H}) .{ }^{13} \mathrm{C} \mathrm{NMR}\left(150 \mathrm{MHz}, \mathrm{CDCl}_{3}\right): \delta 148.6,135.8,132.6,116.3$, 45.1, 29.7, 20.3, 12.0; HRMS (EI) $\mathrm{m} / \mathrm{z}$ calcd for $\mathrm{C}_{9} \mathrm{H}_{12} \mathrm{~N}_{2}[\mathrm{H}]^{+}:$151.1235, found : 151.1239.

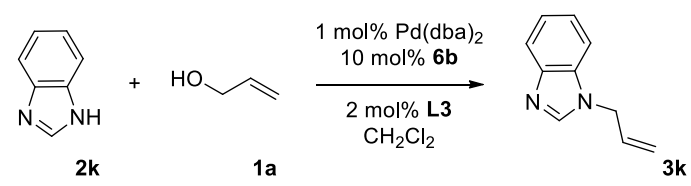

Benzimidazole ( $82 \mathrm{mg}, 0.7 \mathrm{mmol}, 1$ equiv), allyl alcohol (2-propenol, $72 \mu \mathrm{L}, 1.1 \mathrm{mmol}, 1.5$ equiv), L3 (4.9 $\mathrm{mg}, 0.014 \mathrm{mmol}, 2 \mathrm{~mol} \%$ ), pentafluorophenol (13 $\mu \mathrm{L}, 0.07 \mathrm{mmol}, 0.1$ equiv) and bis(dibenzylideneacetone)palladium(0) (4 $\mathrm{mg}, 0.007 \mathrm{mmol}, 1 \mathrm{~mol} \%)$ were dissolved in dichloromethane $(0.7 \mathrm{~mL}, 1 \mathrm{M})$ in a $5 \mathrm{~mL}$ sealed v-vial under $\mathrm{N}_{2}$ atmosphere. After stirring for $6 \mathrm{~h}$ at $30{ }^{\circ} \mathrm{C}$, volatiles were removed on a rotary evaporator. Purification by flash chromatography on silica gel with dichloromethane/methanol (10:1) afforded product, $3 \mathbf{k}(107 \mu \mathrm{L}, 97 \%)$ as a pale yellow oil. ${ }^{1} \mathrm{H}$ $\operatorname{NMR}\left(600 \mathrm{MHz}, \mathrm{CDCl}_{3}\right): \delta 7.92(\mathrm{~s}, 1 \mathrm{H}), 7.83(\mathrm{t}, J=6 \mathrm{~Hz}, J=2.4 \mathrm{~Hz}, 1 \mathrm{H}), 7.39(\mathrm{t}, J=6 \mathrm{~Hz}, J=2.4$ $\mathrm{Hz}, 1 \mathrm{H}), 7.30(\mathrm{~m}, 2 \mathrm{H}), 6.05-5.99(\mathrm{~m}, 1 \mathrm{H}), 5.32(\mathrm{~d}, J=10.8 \mathrm{~Hz}, 1 \mathrm{H}), 5.22(\mathrm{~d}, J=16.8 \mathrm{~Hz}, 1 \mathrm{H}), 4.80(\mathrm{~d}$, $J=5.4 \mathrm{~Hz}, 2 \mathrm{H}) .{ }^{13} \mathrm{C} \mathrm{NMR}\left(150 \mathrm{MHz}, \mathrm{CDCl}_{3}\right): \delta 142.7,131.7,122.8,122.1,120.2,118.5,109.8,47.3$, 29.5; HRMS (EI) $\mathrm{m} / \mathrm{z}$ calcd for $\mathrm{C}_{10} \mathrm{H}_{10} \mathrm{~N}_{2}[\mathrm{Na}]^{+}: 181.0742$, found : 181.0691 .

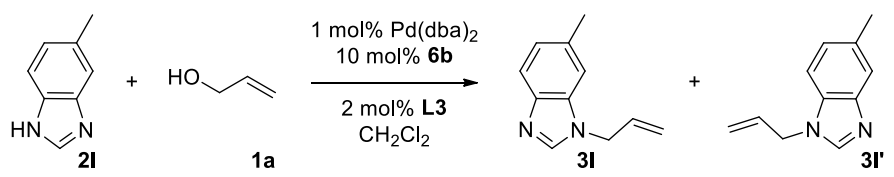

5-Methylbenzimidazole(79 mg, $0.7 \mathrm{mmol}, 1$ equiv), allyl alcohol (2-propenol, $72 \mu \mathrm{L}, 1.1 \mathrm{mmol}, 1.5$ equiv), L3 (4.9 mg, $0.014 \mathrm{mmol}, 2 \mathrm{~mol} \%$ ), pentafluorophenol (13 $\mu \mathrm{L}, 0.07 \mathrm{mmol}, 0.1$ equiv) and bis(dibenzylideneacetone)palladium $(0) \quad(4 \mathrm{mg}, 0.007 \mathrm{mmol}, 1 \mathrm{~mol} \%)$ were dissolved in dichloromethane $(0.7 \mathrm{~mL}, 1 \mathrm{M})$ in a $5 \mathrm{~mL}$ sealed v-vial under $\mathrm{N}_{2}$ atmosphere. After stirring for $6 \mathrm{~h}$ at $30{ }^{\circ} \mathrm{C}$, volatiles were removed on a rotary evaporator. Purification by flash chromatography on silica gel with dichloromethane/methanol (10:1) afforded a mixture of products containing $\mathbf{3 l}$ and $\mathbf{3 l}$ ' in a 1:1 ratio (by $\left.\mathrm{H}^{1} \mathrm{NMR}\right)(107 \mu \mathrm{L}, 97 \%)$ as a pale yellow oil.

$3 \mathbf{l}$ and 3' (1-allyl-6-methyl-1H-benzo[d]imidazole, and 1-allyl-5-methyl-1H-benzo[d]imidazole):

${ }^{1} \mathrm{H}$ NMR $\left(600 \mathrm{MHz}, \mathrm{CDCl}_{3}\right): \delta 7.82(\mathrm{~d}, J=10.8 \mathrm{~Hz}, 1 \mathrm{H}), 7.68(\mathrm{t}, J=8.4 \mathrm{~Hz}, J=46.2 \mathrm{~Hz}, 1 \mathrm{H}), 7.24$ $(\mathrm{t}, J=8.4 \mathrm{~Hz}, J=52.2 \mathrm{~Hz}, 1 \mathrm{H}), 7.11(\mathrm{t}, J=6.6 \mathrm{~Hz}, 1 \mathrm{H}), 6.01-5.94(\mathrm{~m}, 1 \mathrm{H}), 5.28(\mathrm{dd}, J=9.6 \mathrm{~Hz}, J=$ $3.6 \mathrm{~Hz}, 1 \mathrm{H}), 5.17(\mathrm{~d}, J=15.6 \mathrm{~Hz}, 1 \mathrm{H}), 4.72(\mathrm{t}, J=4.8 \mathrm{~Hz}, 2 \mathrm{H}), 2.48(\mathrm{~s}, J=4.8 \mathrm{~Hz}, 1 \mathrm{H}) .{ }^{13} \mathrm{C}$ NMR $(150$ $\left.\mathrm{MHz}, \mathrm{CDCl}_{3}\right): \delta 144.1,142.7,142.3,141.9,134.0,132.8,131.9,131.8,131.7,124.3,123.7,120.0$, 119.7, 118.3, 118.3, 109.6, 109.3, 47.3, 47.1, 21.7, 21.4; HRMS (EI) $\mathrm{m} / \mathrm{z}$ calcd for $\mathrm{C}_{11} \mathrm{H}_{13} \mathrm{~N}_{2}[\mathrm{H}]^{+}$: 173.1079, found : 173.1087 . 


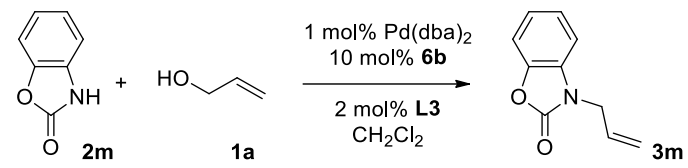

2-Benzoxazolinone (94 mg, $0.7 \mathrm{mmol}, 1$ equiv), allyl alcohol (2-propenol, $72 \mu \mathrm{L}, 1.1 \mathrm{mmol}, 1.5$ equiv), L3 (4.9 mg, $0.014 \mathrm{mmol}, 2 \mathrm{~mol} \%)$, pentafluorophenol (13 $\mu \mathrm{L}, 0.07 \mathrm{mmol}, 0.1$ equiv) and bis(dibenzylideneacetone)palladium $(0)(4 \mathrm{mg}, 0.007 \mathrm{mmol}, 1 \mathrm{~mol} \%)$ were dissolved in dichloromethane $(0.7 \mathrm{~mL}, 1 \mathrm{M})$ in a $5 \mathrm{~mL}$ sealed v-vial under $\mathrm{N}_{2}$ atmosphere. After stirring for $24 \mathrm{~h}$ at $30{ }^{\circ} \mathrm{C}$, volatiles were removed on a rotary evaporator. Purification by flash chromatography on silica gel with dichloromethane/methanol (30:1) afforded product, 3m (113 $\mu \mathrm{L}, 92 \%)$ as a colorless oil. ${ }^{1} \mathrm{H}$ NMR (600 $\left.\mathrm{MHz}, \mathrm{CDCl}_{3}\right): \delta 7.18(\mathrm{~d}, J=7.8 \mathrm{~Hz}, 1 \mathrm{H}), 7.15(\mathrm{td}, J=7.8 \mathrm{~Hz}, J=0.6 \mathrm{~Hz}, 1 \mathrm{H}), 7.10(\mathrm{td}, J=7.2 \mathrm{~Hz}, J$ $=0.6 \mathrm{~Hz}, 1 \mathrm{H}), 6.96(\mathrm{~d}, J=7.8 \mathrm{~Hz}, 1 \mathrm{H}), 5.91-5.85(\mathrm{~m}, 1 \mathrm{H}), 5.30(\mathrm{~d}, J=6 \mathrm{~Hz}, 1 \mathrm{H}), 5.27(\mathrm{~s}, 1 \mathrm{H}), 4.43(\mathrm{~d}$, $J=6 \mathrm{~Hz}, 2 \mathrm{H}) .{ }^{13} \mathrm{C}$ NMR $\left(600 \mathrm{MHz}, \mathrm{CDCl}_{3}\right): \delta 154.1,142.5,130.8,130.4,123.6,122.3,118.5,109.8$, 108.7, 44.4; HRMS (EI) $\mathrm{m} / \mathrm{z}$ calcd for $\mathrm{C}_{10} \mathrm{H}_{9} \mathrm{NO}_{2}[\mathrm{Na}]^{+}:$: 198.0531 , found : 198.0518 .

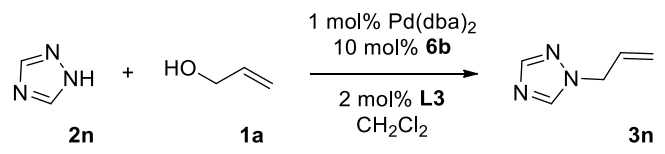

1,2,4-Triazole (48 mg, $0.7 \mathrm{mmol}, 1$ equiv), allyl alcohol (2-propenol, $72 \mu \mathrm{L}, 1.1 \mathrm{mmol}, 1.5$ equiv), L3 (4.9 mg, $0.014 \mathrm{mmol}, 2 \mathrm{~mol} \%$ ), pentafluorophenol (13 $\mu \mathrm{L}, 0.07 \mathrm{mmol}, 0.1$ equiv) and bis(dibenzylideneacetone)palladium(0) (4 $\mathrm{mg}, 0.007 \mathrm{mmol}, 1 \mathrm{~mol} \%)$ were dissolved in dichloromethane $(0.7 \mathrm{~mL}, 1 \mathrm{M})$ in a $5 \mathrm{~mL}$ sealed v-vial under $\mathrm{N}_{2}$ atmosphere. After stirring for $6 \mathrm{~h}$ at $30{ }^{\circ} \mathrm{C}$, volatiles were removed on a rotary evaporator. Purification by flash chromatography on silica gel with dichloromethane/methanol (10:1) afforded product, 3n $(74 \mu \mathrm{L}, 97 \%)$ as a colorless oil. The measured NMR spectra agreed with the literature values. ${ }^{\left[{ }^{1} 1\right]}{ }^{1} \mathrm{H}$ NMR $\left(600 \mathrm{MHz}, \mathrm{CDCl}_{3}\right): \delta 8.07(\mathrm{~d}, J$ $=5.4 \mathrm{~Hz}, 1 \mathrm{H}), 7.93(\mathrm{~d}, J=5.4 \mathrm{~Hz}, 1 \mathrm{H}), 6.02-5.96(\mathrm{~m}, 1 \mathrm{H}), 5.34(\mathrm{dd}, J=10.2 \mathrm{~Hz}, J=4.2 \mathrm{~Hz}, 1 \mathrm{H}), 5.27$ $(\mathrm{dd}, J=16.8 \mathrm{~Hz}, J=4.8 \mathrm{~Hz}, 1 \mathrm{H}), 4.78(\mathrm{t}, J=5.4 \mathrm{~Hz}, 2 \mathrm{H}) .{ }^{13} \mathrm{C} \mathrm{NMR}\left(150 \mathrm{MHz}, \mathrm{CDCl}_{3}\right): \delta 151.9,142.7$, $131.1,119.8,52.0$.

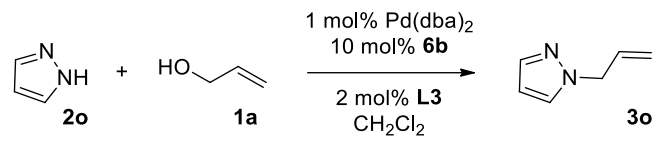

Pyrazole (48 mg, $0.7 \mathrm{mmol}, 1$ equiv), allyl alcohol (2-propenol, $72 \mu \mathrm{L}, 1.1 \mathrm{mmol}, 1.5$ equiv), L3 (4.9 $\mathrm{mg}, \quad 0.014 \mathrm{mmol}, 2 \mathrm{~mol} \%)$, pentafluorophenol (13 $\mu \mathrm{L}, 0.07 \mathrm{mmol}, 0.1$ equiv) and bis(dibenzylideneacetone)palladium(0) (4 $\mathrm{mg}, 0.007 \mathrm{mmol}, 1 \mathrm{~mol} \%)$ were dissolved in dichloromethane $(0.7 \mathrm{~mL}, 1 \mathrm{M})$ in a $5 \mathrm{~mL}$ sealed v-vial at $\mathrm{N}_{2}$ atmosphere. After stirring for $6 \mathrm{~h}$ at $30^{\circ} \mathrm{C}$, volatiles were removed on a rotary evaporator. Purification by flash chromatography on silica gel with dichloromethane/methanol (10:1) afforded product, 3o $(71 \mu \mathrm{L}, 94 \%)$ as a colorless oil. The measured NMR spectra agreed with the literature values. ${ }^{[\mathrm{S} 6]}{ }^{1} \mathrm{H} \mathrm{NMR}\left(600 \mathrm{MHz}, \mathrm{CDCl}_{3}\right): \delta 8.48(\mathrm{~s}, 1 \mathrm{H}), 7.35$ (s, $1 \mathrm{H}), 6.22(\mathrm{~s}, 1 \mathrm{H}), 6.00-5.94(\mathrm{~m}, 1 \mathrm{H}), 5.22(\mathrm{~d}, J=10.2 \mathrm{~Hz}, 1 \mathrm{H}), 5.16(\mathrm{~d}, J=17.4 \mathrm{~Hz}, 1 \mathrm{H}), 4.71(\mathrm{~d}, J=$ $5.4 \mathrm{~Hz}, 2 \mathrm{H}) .{ }^{13} \mathrm{C}$ NMR $\left(150 \mathrm{MHz}, \mathrm{CDCl}_{3}\right): \delta 139.3,133.0,128.9,118.4,105.7,54.5$.

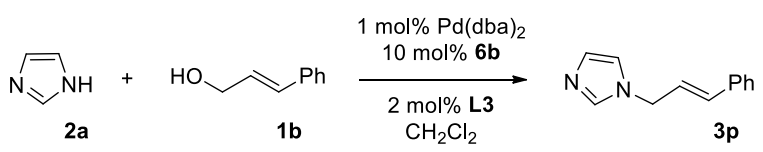

Imidazole (48 mg, 0.7 mmol, 1 equiv), cinnamyl alcohol (3-phenylprop-2-en-1-ol, $137 \mu \mathrm{L}, 1.1 \mathrm{mmol}$, 
1.5 equiv), L3 (4.9 mg, $0.014 \mathrm{mmol}, 2 \mathrm{~mol} \%)$, pentafluorophenol (13 $\mu \mathrm{L}, 0.07 \mathrm{mmol}, 0.1$ equiv) and bis(dibenzylideneacetone)palladium(0) (4 $\mathrm{mg}, 0.007 \mathrm{mmol}, 1 \mathrm{~mol} \%)$ were dissolved in dichloromethane $(0.7 \mathrm{~mL}, 1 \mathrm{M})$ in a $5 \mathrm{~mL}$ sealed v-vial under $\mathrm{N}_{2}$ atmosphere. After stirring for $6 \mathrm{~h}$ at $30{ }^{\circ} \mathrm{C}$, volatiles were removed on a rotary evaporator. Purification by flash chromatography on silica gel with dichloromethane/methanol (10:1) afforded product, 3p (124 $\mu \mathrm{L}, 96 \%)$ as a colorless oil. The measured NMR spectra agreed with the literature values. ${ }^{[\mathrm{S} 2]}{ }^{1} \mathrm{H} \mathrm{NMR}\left(600 \mathrm{MHz}, \mathrm{CDCl}_{3}\right): \delta 7.53(\mathrm{~s}, 1 \mathrm{H})$, $7.36(\mathrm{~d}, J=7.2 \mathrm{~Hz}, 2 \mathrm{H}), 7.34(\mathrm{t}, J=7.8 \mathrm{~Hz}, 2 \mathrm{H}), 7.28(\mathrm{t}, J=7.2 \mathrm{~Hz}, 2 \mathrm{H}), 7.09(\mathrm{~s}, 1 \mathrm{H}), 6.96(\mathrm{~s}, 1 \mathrm{H})$, $6.53(\mathrm{~d}, J=15.6 \mathrm{~Hz}, 1 \mathrm{H}), 6.30-6.25(\mathrm{~m}, 1 \mathrm{H}), 4.70(\mathrm{~d}, J=6 \mathrm{~Hz}, 1 \mathrm{H}) .{ }^{13} \mathrm{C}$ NMR $\left(150 \mathrm{MHz}, \mathrm{CDCl}_{3}\right): \delta$ 137.0, 135.6, 133.6, 129.6, 128.6, 128.2, 126.5, 123.6, 118.9, 48.9; HRMS (EI) m/z calcd for $\mathrm{C}_{12} \mathrm{H}_{13} \mathrm{~N}_{2}$ $[\mathrm{H}]^{+}: 185.1079$, found $: 185.1059$.

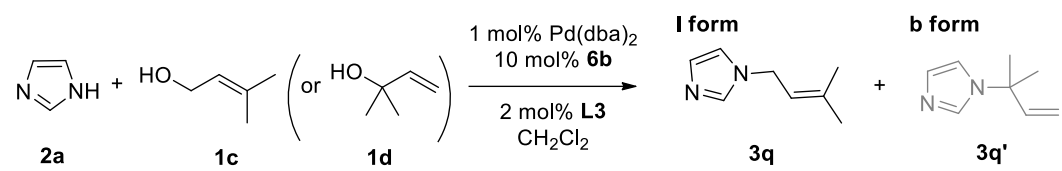

3-Methyl-2-buten-1-ol (1c): Imidazole (48 mg, $0.7 \mathrm{mmol}, 1$ equiv), 3-methyl-2-buten-1-ol (107 $\mu \mathrm{L}$, $1.1 \mathrm{mmol}, 1.5$ equiv), L3 (4.9 mg, $0.014 \mathrm{mmol}, 2 \mathrm{~mol} \%)$, pentafluorophenol ( $13 \mu \mathrm{L}, 0.07 \mathrm{mmol}, 0.1$ equiv) and bis(dibenzylideneacetone)palladium(0) $(4 \mathrm{mg}, 0.007 \mathrm{mmol}, 1 \mathrm{~mol} \%$ ) were dissolved in dichloromethane $(0.7 \mathrm{~mL}, 1 \mathrm{M})$ in a $5 \mathrm{~mL}$ sealed v-vial under $\mathrm{N}_{2}$ atmosphere. After stirring for $6 \mathrm{~h}$ at $30{ }^{\circ} \mathrm{C}$, volatiles were removed on a rotary evaporator. Purification by flash chromatography on silica gel with dichloromethane/methanol (10:1) afforded product, $\mathbf{3 q}(86 \mu \mathrm{L}, 90 \%)$ as a colorless oil.

2-Methyl-3-buten-2-ol (1d):Imidazole (48 mg, $0.7 \mathrm{mmol}, 1$ equiv), 2-methyl-3-buten-2-ol (107 $\mu \mathrm{L}$, $1.1 \mathrm{mmol}, 1.5$ equiv), L3 (4.9 mg, $0.014 \mathrm{mmol}, 2 \mathrm{~mol} \%)$, pentafluorophenol ( $13 \mu \mathrm{L}, 0.07 \mathrm{mmol}, 0.1$ equiv) and bis(dibenzylideneacetone)palladium(0) $(4 \mathrm{mg}, 0.007 \mathrm{mmol}, 1 \mathrm{~mol} \%)$ were dissolved in dichloromethane $(0.7 \mathrm{~mL}, 1 \mathrm{M})$ in a $5 \mathrm{~mL}$ sealed v-vial under $\mathrm{N}_{2}$ atmosphere. After stirring for $6 \mathrm{~h}$ at $30{ }^{\circ} \mathrm{C}$, volatiles were removed on a rotary evaporator. Purification by flash chromatography on silica gel with dichloromethane/methanol (10:1) afforded product, 3q (94 $\mu \mathrm{L}, 99 \%)$ as a colorless oil.

${ }^{1} \mathrm{H}$ NMR $\left(600 \mathrm{MHz}, \mathrm{CDCl}_{3}\right): \delta 7.43(\mathrm{~s}, 1 \mathrm{H}), 7.01(\mathrm{~s}, 1 \mathrm{H}), 6.85(\mathrm{~s}, 1 \mathrm{H}), 5.34(\mathrm{t}, J=7.8 \mathrm{~Hz}, 1 \mathrm{H}), 4.48(\mathrm{~d}$, $J=7.2 \mathrm{~Hz}, 2 \mathrm{H}), 1.75(\mathrm{~s}, 3 \mathrm{H}), 1.73(\mathrm{~s}, 3 \mathrm{H}) .{ }^{13} \mathrm{C} \mathrm{NMR}\left(150 \mathrm{MHz}, \mathrm{CDCl}_{3}\right): \delta 137.9,136.6,129.2,118.6$, 118.5, 44.5, 25.5, 17.8; HRMS (EI) $\mathrm{m} / \mathrm{z}$ calcd for $\mathrm{C}_{8} \mathrm{H}_{12} \mathrm{~N}_{2}[\mathrm{Na}]^{+}: 159.0898$, found : 159.0889 .

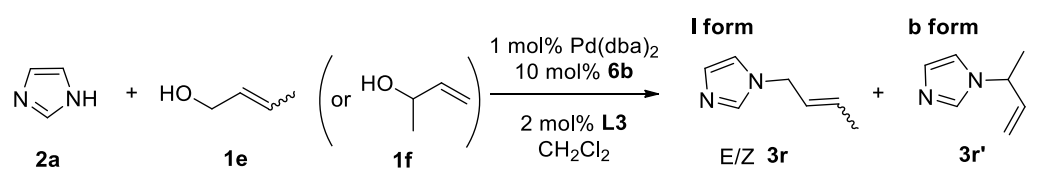

Crotyl alcohol (1e): Imidazole (48 mg, $0.7 \mathrm{mmol}, 1$ equiv), crotyl alcohol (2-Butenol, $77 \mu \mathrm{L}$, $1.1 \mathrm{mmol}, 1.5$ equiv), $\mathrm{L} 3$ ( $4.9 \mathrm{mg}, 0.014 \mathrm{mmol}, 2 \mathrm{~mol} \%)$, pentafluorophenol ( $13 \mu \mathrm{L}, 0.07 \mathrm{mmol}, 0.1$ equiv) and bis(dibenzylideneacetone)palladium(0) ( $4 \mathrm{mg}, 0.007 \mathrm{mmol}, 1 \mathrm{~mol} \%)$ were dissolved in dichloromethane $(0.7 \mathrm{~mL}, 1 \mathrm{M})$ in a $5 \mathrm{~mL}$ sealed v-vial under $\mathrm{N}_{2}$ atmosphere. After stirring for $6 \mathrm{~h}$ at $30{ }^{\circ} \mathrm{C}$, volatiles were removed on a rotary evaporator. Purification by flash chromatography on silica gel with dichloromethane/methanol (10:1) afforded a mixture of products containing $\mathbf{3 r}$ and $\mathbf{3} \mathbf{r}$ ' in a 83:17 ratio (by $\mathrm{H}^{1} \mathrm{NMR}$ ) ( $85 \mathrm{mg}, 98 \%$, linear/branch ratio was obtained 5/1) as a colorless oil.

3-But-2-ol (1f): Imidazole (48 mg, $0.7 \mathrm{mmol}, 1$ equiv), 3-but-2-ol ( $77 \mu \mathrm{L}, 1.1 \mathrm{mmol}, 1.5$ equiv), L3 (4.9 $\mathrm{mg}, 0.014 \mathrm{mmol}, 2 \mathrm{~mol} \%$ ), pentafluorophenol $(13 \mu \mathrm{L}, 0.07 \mathrm{mmol}, 0.1$ equiv) and bis(dibenzylideneacetone)palladium(0) (4 $\mathrm{mg}, 0.007 \mathrm{mmol}, 1 \mathrm{~mol} \%)$ were dissolved in dichloromethane $(0.7 \mathrm{~mL}, 1 \mathrm{M})$ in a $5 \mathrm{~mL}$ sealed v-vial under $\mathrm{N}_{2}$ atmosphere. After stirring for $6 \mathrm{~h}$ at $30{ }^{\circ} \mathrm{C}$, volatiles were removed on a rotary evaporator. Purification by flash chromatography on silica gel with dichloromethane/methanol (10:1) afforded a mixture of products containing $\mathbf{3 r}$ and $\mathbf{3} \mathbf{r}^{\prime}$ in a 88:12 ratio (by $\mathrm{H}^{1} \mathrm{NMR}$ ) (86 $\mathrm{mg}, 99 \%$, linear/branch ratio was obtained 7/1) as a colorless oil. The 
measured NMR spectra agreed with the literature values. ${ }^{\left[{ }^{[3]}\right]}$

Linear form (3r): $(E)$-form ${ }^{1} \mathrm{H}$ NMR $\left(\mathrm{CDCl}_{3}\right): \delta 7.46(\mathrm{~s}, 1 \mathrm{H}), 7.05(\mathrm{~s}, 1 \mathrm{H}), 6.89(\mathrm{~s}, 1 \mathrm{H}), 5.73-5.67(\mathrm{~m}$, $1 \mathrm{H}), 5.61-5.57(\mathrm{~m}, 1 \mathrm{H}), 4.47(\mathrm{~d}, J=6 \mathrm{~Hz}, 2 \mathrm{H}), 1.74(\mathrm{~d}, J=6.6 \mathrm{~Hz}, 3 \mathrm{H})$. (Z)-form ${ }^{1} \mathrm{H}$ NMR $(600 \mathrm{MHz}$, $\left.\mathrm{CDCl}_{3}\right): \delta 7.48(\mathrm{~s}, 1 \mathrm{H}), 7.05(\mathrm{~s}, 1 \mathrm{H}), 6.90(\mathrm{~s}, 1 \mathrm{H}), 5.82-5.77(\mathrm{~m}, 1 \mathrm{H}), 5.62-5.57(\mathrm{~m}, 1 \mathrm{H}), 4.59(\mathrm{~d}, J=$ $7.2 \mathrm{~Hz}, 2 \mathrm{H}), 1.77(\mathrm{~d}, J=7.2 \mathrm{~Hz}, 3 \mathrm{H}) .{ }^{13} \mathrm{C} \mathrm{NMR}\left(150 \mathrm{MHz}, \mathrm{CDCl}_{3}\right): \delta 136.6,136.5,130.4,129.2,129.0$, $128.9,125.3,124.1,118.6,118.5,48.7,43.1,17.3,12.7$.

Branched form (3r'): ${ }^{1} \mathrm{H}$ NMR (600 MHz, $\left.\mathrm{CDCl}_{3}\right): \delta 7.55$ (s, 1H), $7.08(\mathrm{~s}, 1 \mathrm{H}), 6.95(\mathrm{~s}, 1 \mathrm{H}), 6.00-5.94$ $(\mathrm{m}, 1 \mathrm{H}), 5.23(\mathrm{~d}, J=10.2 \mathrm{~Hz}, 1 \mathrm{H}), 5.13(\mathrm{~d}, J=17.4 \mathrm{~Hz}, 1 \mathrm{H}), 4.77(\mathrm{~m}, 1 \mathrm{H}), 1.60(\mathrm{~d}, J=6.6 \mathrm{~Hz}, 1 \mathrm{H})$. ${ }^{13} \mathrm{C}$ NMR (150 MHz, $\left.\mathrm{CDCl}_{3}\right): \delta$ 138.0, 135.43, 128.8, 117.1, 116.1, 54.8, 20.4.

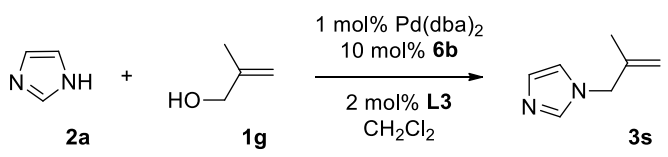

Imidazole (48 mg, $0.7 \mathrm{mmol}, 1$ equiv), 2-methyl-2-propen-1-ol ( $88 \mu \mathrm{L}, 1.1 \mathrm{mmol}, 1.5$ equiv), L3 (4.9mg, $0.014 \mathrm{mmol}, 2 \mathrm{~mol} \%$ ), pentafluorophenol (13 $\mu \mathrm{L}, 0.07 \mathrm{mmol}, 0.1$ equiv) and bis(dibenzylideneacetone)palladium $(0)(4 \mathrm{mg}, 0.007 \mathrm{mmol}, 1 \mathrm{~mol} \%)$ were dissolved in dichloromethane $(0.7 \mathrm{~mL}, 1 \mathrm{M})$ in a $5 \mathrm{~mL}$ sealed v-vial under $\mathrm{N}_{2}$ atmosphere. After stirring for $6 \mathrm{~h}$ at $30^{\circ} \mathrm{C}$, volatiles were removed on a rotary evaporator. Purification by flash chromatography on silica gel with dichloromethane/methanol (10:1) afforded product, $3 \mathbf{s}(84 \mu \mathrm{L}, 98 \%)$ as a colorless oil. The measured NMR spectra agreed with the literature values. ${ }^{[\mathrm{S} 4]}{ }^{1} \mathrm{H}$ NMR $\left(600 \mathrm{MHz}, \mathrm{CDCl}_{3}\right): \delta 7.44(\mathrm{~s}, 1 \mathrm{H}), 7.04$ (s, $1 \mathrm{H}), 6.86(\mathrm{~s}, 1 \mathrm{H}), 4.94(\mathrm{~s}, 1 \mathrm{H}), 4.78(\mathrm{~s}, 1 \mathrm{H}), 4.43(\mathrm{~s}, 1 \mathrm{H}), 1.66(\mathrm{~s}, 3 \mathrm{H}),{ }^{13} \mathrm{C} \mathrm{NMR}\left(150 \mathrm{MHz}, \mathrm{CDCl}_{3}\right): \delta$ $140.4,137.4,129.3,119.1,113.8,53.0,19.5$.

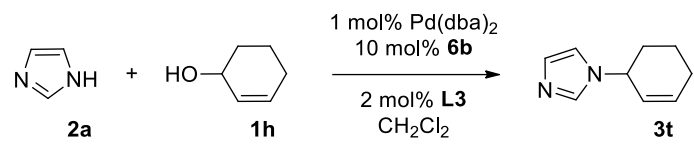

Imidazole (48 mg, 0.7 mmol, 1 equiv), 2-cyclohexen-1-ol (103 $\mu \mathrm{L}, 1.1 \mathrm{mmol}, 1.5$ equiv), L3 (4.9 mg, $0.014 \mathrm{mmol}, 2 \mathrm{~mol} \%)$, pentafluorophenol (13 $\mu \mathrm{L}, 0.07 \mathrm{mmol}, 0.1$ equiv) and bis(dibenzylideneacetone)palladium $(0) \quad(4 \mathrm{mg}, 0.007 \mathrm{mmol}, 1 \mathrm{~mol} \%)$ were dissolved in dichloromethane $(0.7 \mathrm{~mL}, 1 \mathrm{M})$ in a $5 \mathrm{~mL}$ sealed v-vial under $\mathrm{N}_{2}$ atmosphere. After stirring for $6 \mathrm{~h}$ at $30{ }^{\circ} \mathrm{C}$, volatiles were removed on a rotary evaporator. Purification by flash chromatography on silica gel with dichloromethane/methanol (10:1) afforded product, $3 \mathbf{t}(90 \mu \mathrm{L}, 87 \%)$ as a colorless oil. ${ }^{1} \mathrm{H}$ NMR $\left(600 \mathrm{MHz}, \mathrm{CDCl}_{3}\right): \delta 7.52(\mathrm{~s}, 1 \mathrm{H}), 7.03(\mathrm{~s}, 1 \mathrm{H}), 6.94(\mathrm{~s}, 1 \mathrm{H}), 6.08(\mathrm{~m}, 1 \mathrm{H}), 5.73(\mathrm{dd}, J=9.6 \mathrm{~Hz}, J=$ $2.4 \mathrm{~Hz}, 1 \mathrm{H}), 4.69(\mathrm{~s}, 1 \mathrm{H}), 2.10-1.65(\mathrm{~m}, 6 \mathrm{H}) .{ }^{13} \mathrm{C} \mathrm{NMR}\left(150 \mathrm{MHz}, \mathrm{CDCl}_{3}\right): \delta 136.1,132.7,129.0,125.3$, 117.7, 52.5, 31.7, 24.5, 19.2; HRMS (EI) m/z calcd for $\mathrm{C}_{9} \mathrm{H}_{12} \mathrm{~N}_{2}[\mathrm{Na}]^{+}: 171.0898$, found : 171.0901 .

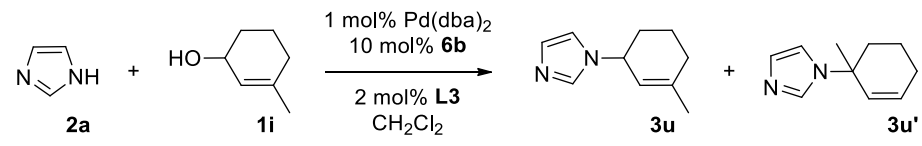

Imidazole (48 mg, 0.7 mmol, 1 equiv), 3-methyl-2-cyclohexen-1-ol (118 $\mu \mathrm{L}, 1.1 \mathrm{mmol}, 1.5$ equivL3 (4.9 mg, $0.014 \mathrm{mmol}, 2 \mathrm{~mol} \%$ ), pentafluorophenol $(13 \mu \mathrm{L}, 0.07 \mathrm{mmol}, 0.1$ equiv) and bis(dibenzylideneacetone)palladium(0) (4 $\mathrm{mg}, 0.007 \mathrm{mmol}, 1 \mathrm{~mol} \%)$ were dissolved in dichloromethane $(0.7 \mathrm{~mL}, 1 \mathrm{M})$ in a $5 \mathrm{~mL}$ sealed v-vial under $\mathrm{N}_{2}$ atmosphere. After stirring for $6 \mathrm{~h}$ at $30{ }^{\circ} \mathrm{C}$, volatiles were removed on a rotary evaporator. Purification by flash chromatography on silica gel with dichloromethane/methanol (10:1) afforded product, $3 \mathbf{u}(85 \mu \mathrm{L}, 75 \%)$ as a colorless oil. ${ }^{1} \mathrm{H}$ NMR $\left(600 \mathrm{MHz}, \mathrm{CDCl}_{3}\right): \delta 7.52(\mathrm{~s}, 1 \mathrm{H}), 7.03(\mathrm{~s}, 1 \mathrm{H}), 6.93(\mathrm{~s}, 1 \mathrm{H}), 5.47(\mathrm{~s}, 1 \mathrm{H}), 4.67(\mathrm{~s}, 1 \mathrm{H}), 2.09$ $1.98(\mathrm{~m}, 4 \mathrm{H}), 1.77(\mathrm{~s}, 3 \mathrm{H}), 1.75-1.65(\mathrm{~m}, 2 \mathrm{H}) .{ }^{13} \mathrm{C} \mathrm{NMR}\left(150 \mathrm{MHz}, \mathrm{CDCl}_{3}\right): \delta 140.6,136.1,128.9$, 119.7, 117.8, 53.1, 31.4, 29.6, 23.6, 19.5; HRMS (EI) $\mathrm{m} / \mathrm{z}$ calcd for $\mathrm{C}_{10} \mathrm{H}_{14} \mathrm{~N}_{2}[\mathrm{Na}]^{+}:$: 185.1055, found : 
185.1052.

\section{References}

(S1) Gagosz, F.; Zard, S. Z. Org, Lett. 2002, 4, 4345-4348.

(S2) Ghosh, R.; Sarkar, A. J. Org. Chem. 2011, 76, 8508-8512.

(S3) Beccalli, E.; Broggini, G.; Contini, A.; Marchi, I. D.; Zecchi, G.; Zoni, C. Tetrahedron: Asymmetry 2004, 15, 3181-3187.

(S4) Bauerlein, P. S.; Gonzlez, I. A.; Weemers, J. J. M.; Lutz, M.; Spek, A. L.; Vogt, D.; Muller, C. Chem. Commun. 2009, 4944-4946.

(S5) Bridger, G.; Kaller, A.; Harwig, C.; Skerlj, R.; Bougucki, D.; Wilson, T. R.; Crawford, J.; McEachern, E. J.; Atsma, B.; Nan, S.; Zhou, Y.; Schols, D.; Smith, C. D.; Fluri, M. R. D. US 2004/0019058 Al

(S6) a) Diez-Barra, E.; Hoz, A. D. L.; Sanchez-Migallon, A.; Tejeda, J. Syn. Commun. 1990, 20, 2849-2853. b) E. Diez-Barra, A. D. L. Hoz, A. Loupy, A. Sanchez-Migallon, Heterocycles 1994, 38, 1367-1374. 


\section{NMR spectra}

${ }^{1} \mathrm{H}$ NMR $\left(600 \mathrm{MHz}, \mathrm{CDCl}_{3}\right)$

$\approx \mathrm{N}_{3 a}^{\mathrm{N}}$

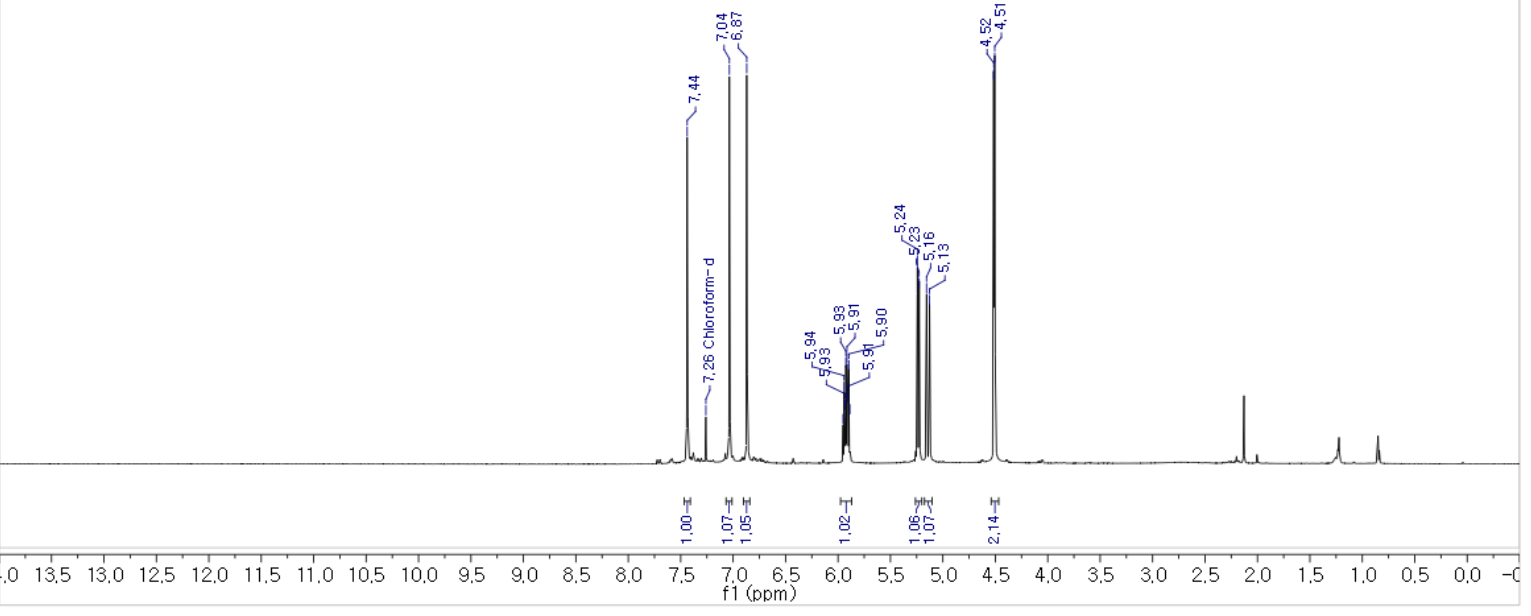

${ }^{13} \mathrm{C}$ NMR (150 MHz, $\mathrm{CDCl}_{3}$ )

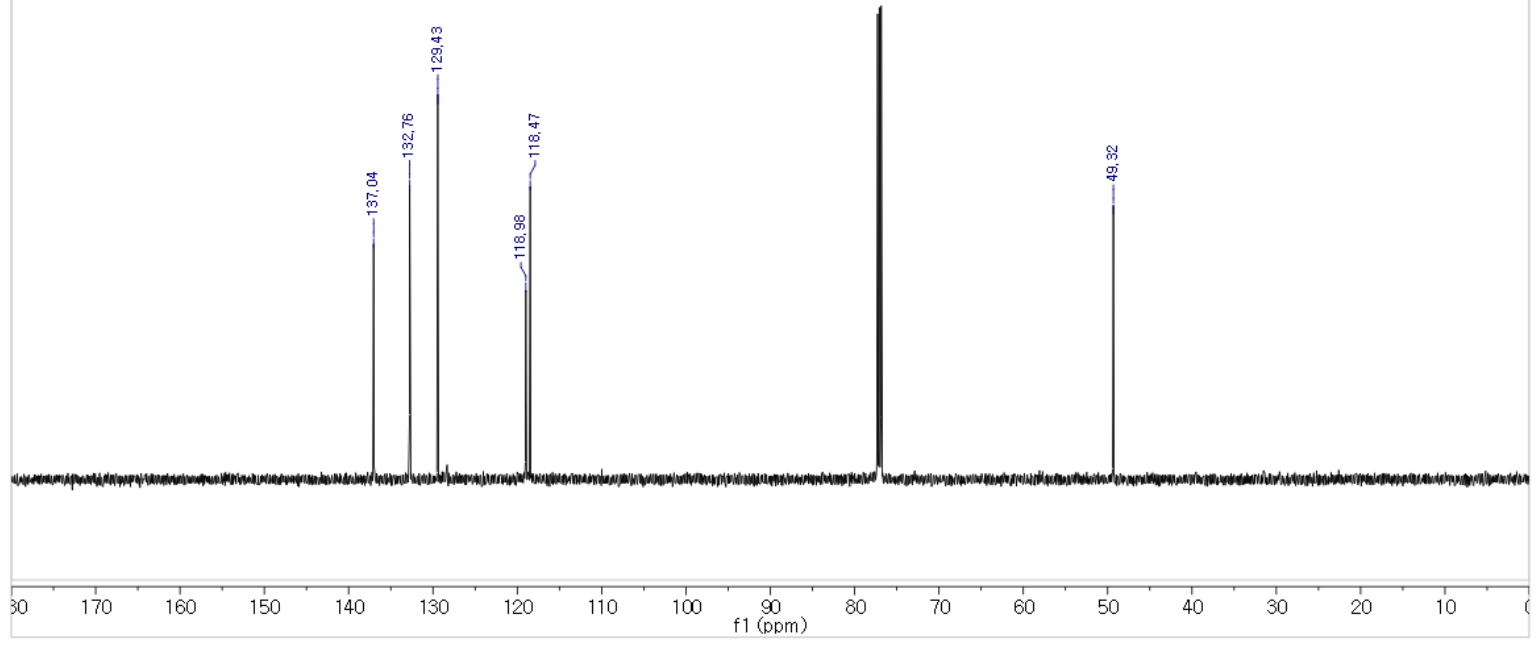


${ }^{1} \mathrm{H}$ NMR $\left(600 \mathrm{MHz}, \mathrm{CDCl}_{3}\right)$
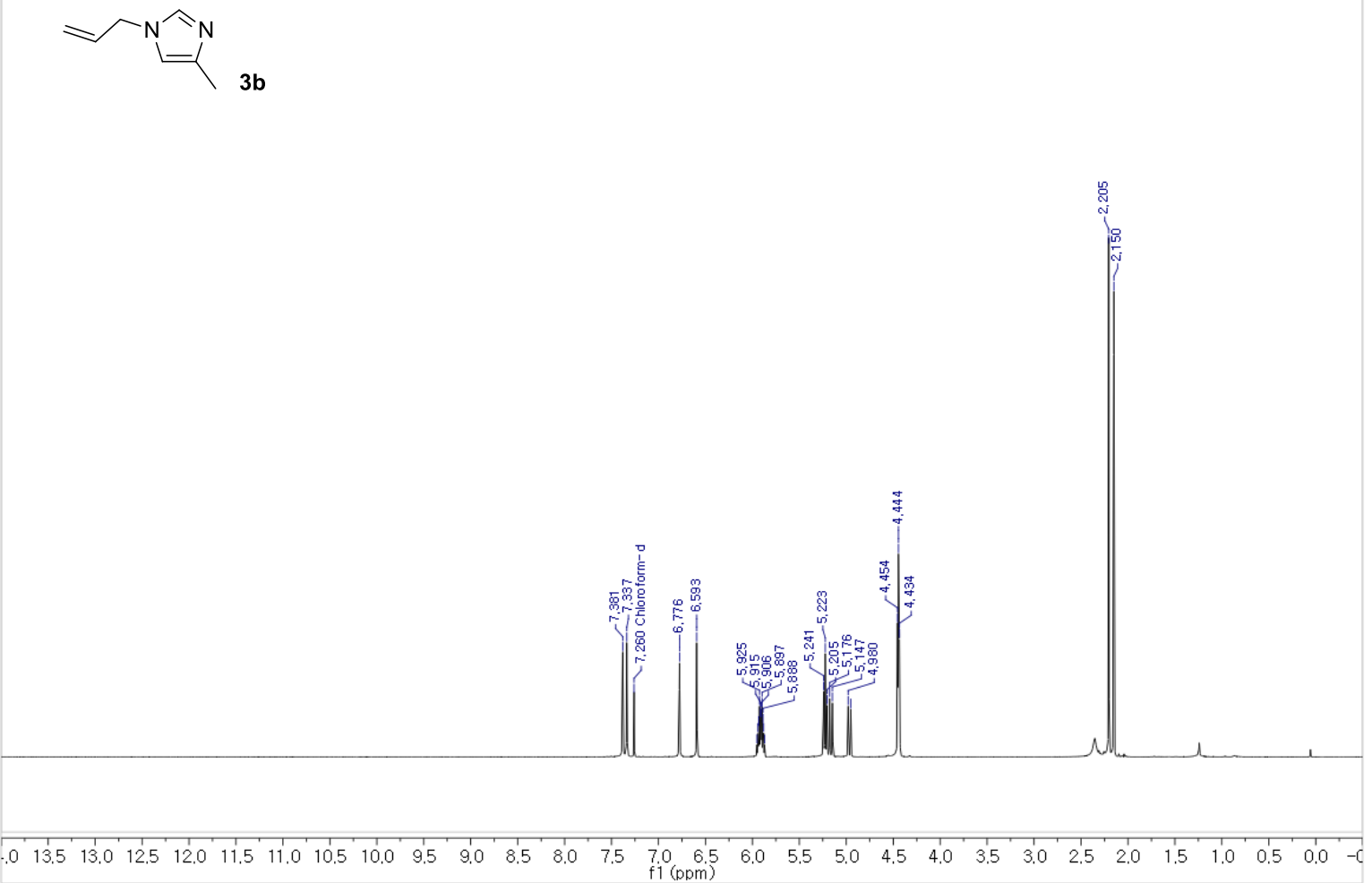

${ }^{13} \mathrm{C}$ NMR (150 MHz, $\mathrm{CDCl}_{3}$ )

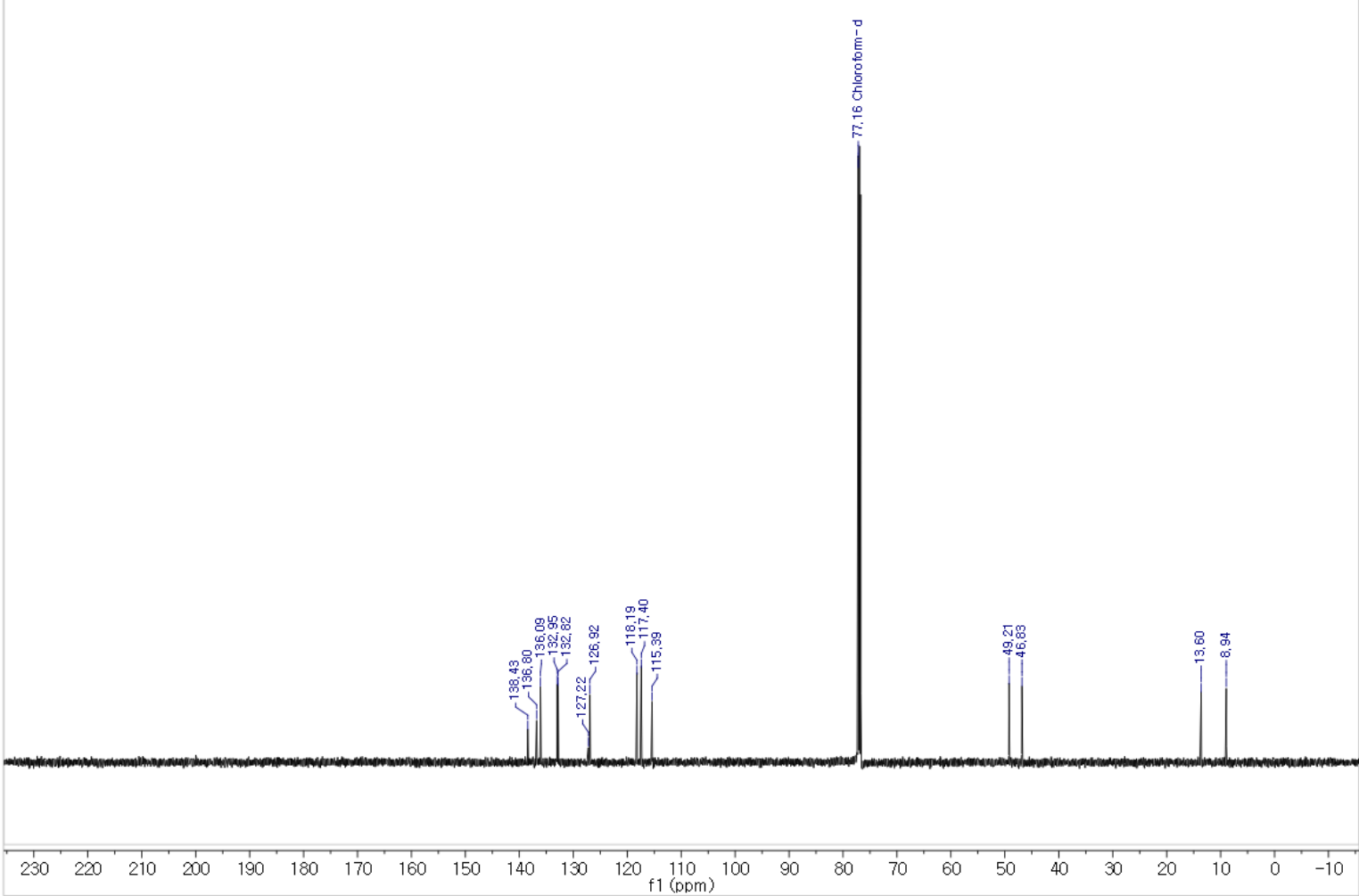


${ }^{1} \mathrm{H}$ NMR $\left(600 \mathrm{MHz}, \mathrm{CDCl}_{3}\right)$

$$
\approx \overbrace{3 \mathrm{C}}^{\mathrm{N}}
$$

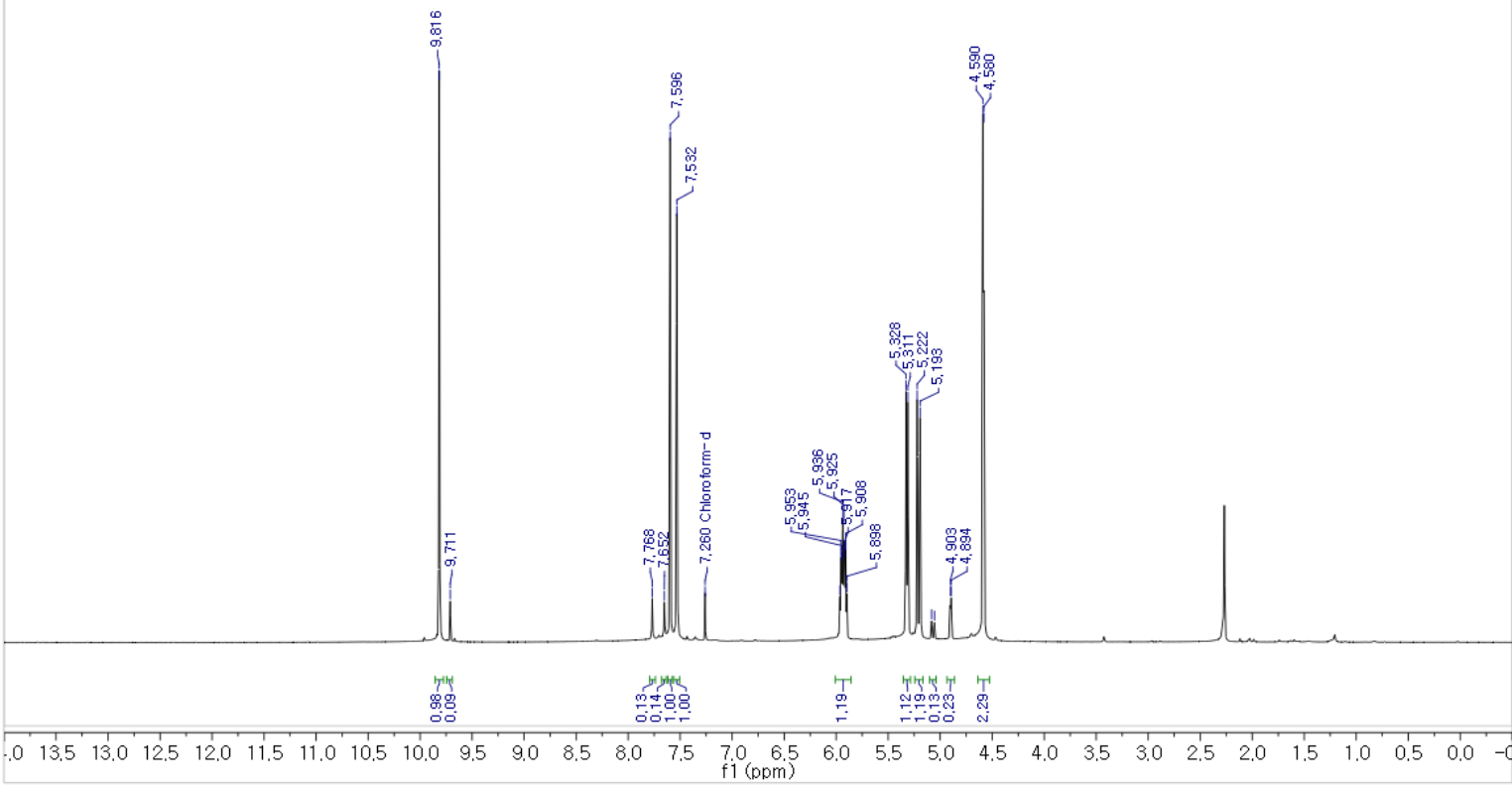

${ }^{13} \mathrm{C}$ NMR $\left(150 \mathrm{MHz}, \mathrm{CDCl}_{3}\right)$

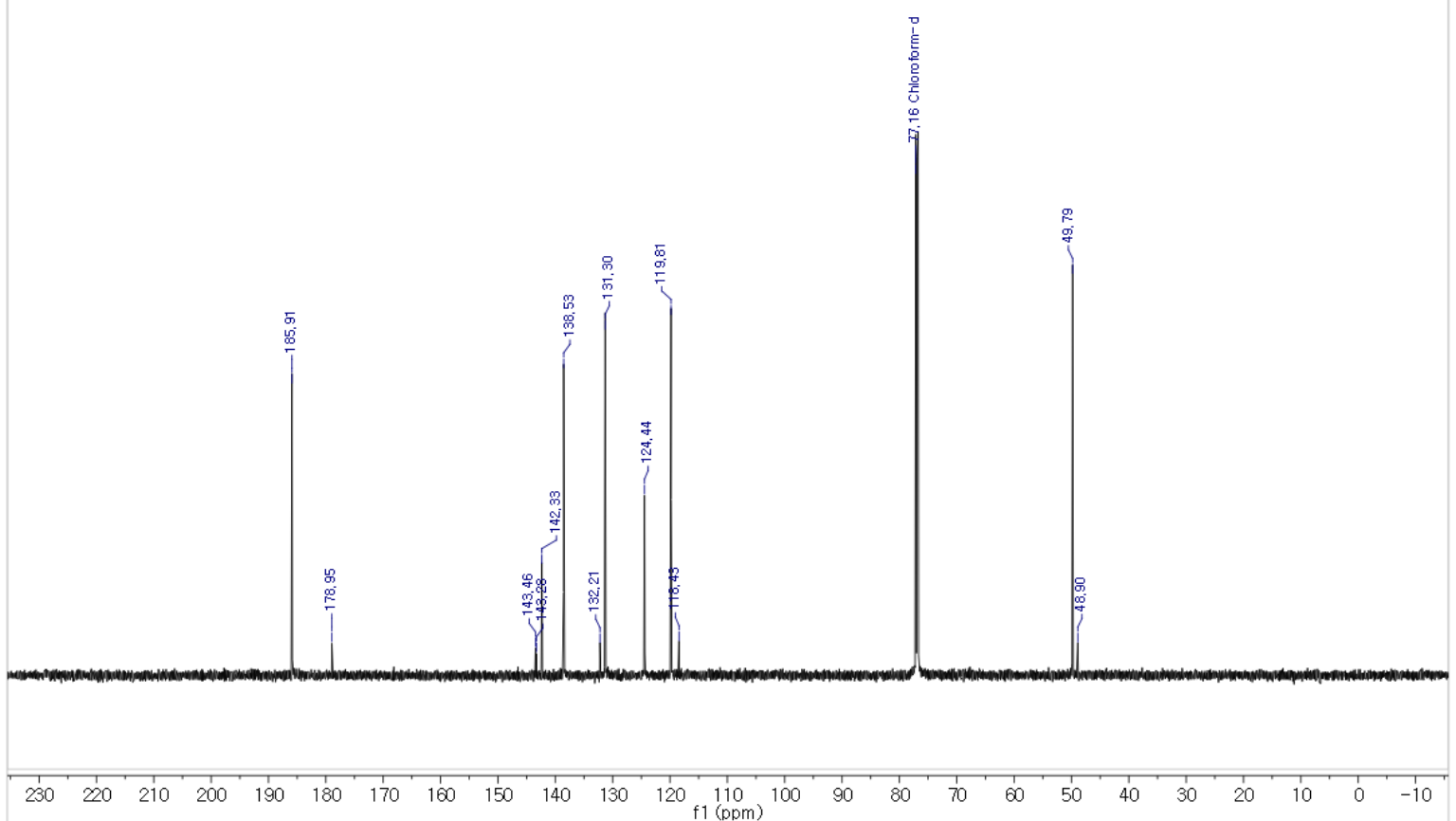


${ }^{1} \mathrm{H} \mathrm{NMR}\left(600 \mathrm{MHz}, \mathrm{CDCl}_{3}\right)$

$$
\approx \overbrace{3 d}^{N} \hat{N}_{N}
$$

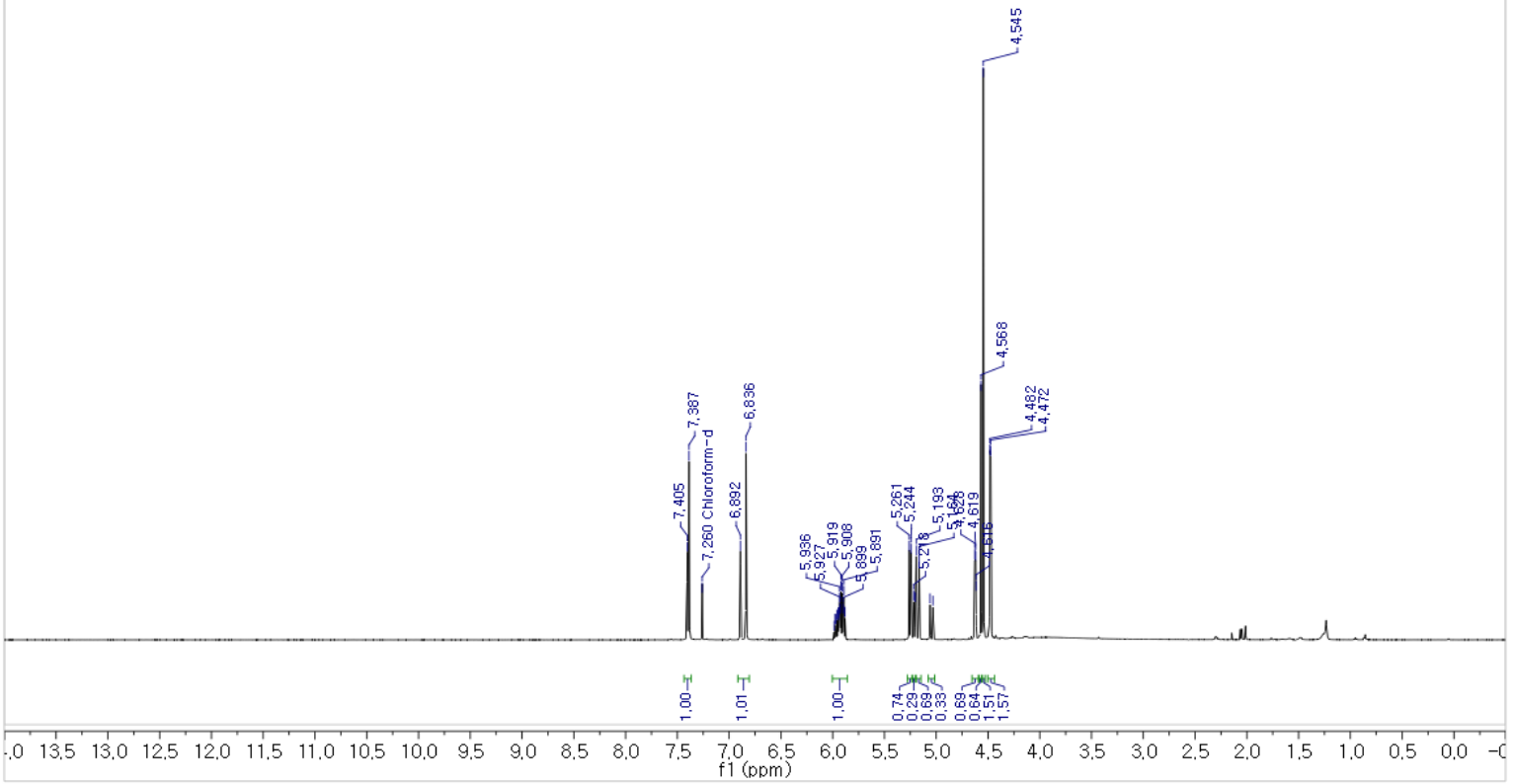

$\left.{ }^{13} \mathrm{C} \mathrm{NMR} \mathrm{(150} \mathrm{MHz,} \mathrm{CDCl}_{3}\right)$

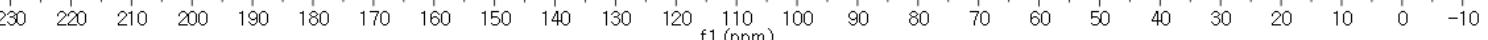


${ }^{1} \mathrm{H}$ NMR $\left(600 \mathrm{MHz}, \mathrm{CDCl}_{3}\right)$

$\approx \gamma_{\mathrm{N}} \hat{\mathrm{CHO}}_{3 e}$

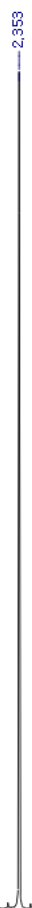

$\begin{array}{llllllllllllllllllllllllllllllllllll}0 & 13.5 & 13.0 & 12.5 & 12.0 & 11.5 & 11.0 & 10.5 & 10.0 & 9.5 & 9.0 & 8.5 & 8.0 & 7.5 & \begin{array}{l}7.0 \\ \text { f1(pm) }\end{array} & 6.0 & 5.5 & 5.0 & 4.5 & 4.0 & 3.5 & 3.0 & 2.5 & 2.0 & 1.5 & 1.0 & 0.5 & 0.0 & -0\end{array}$

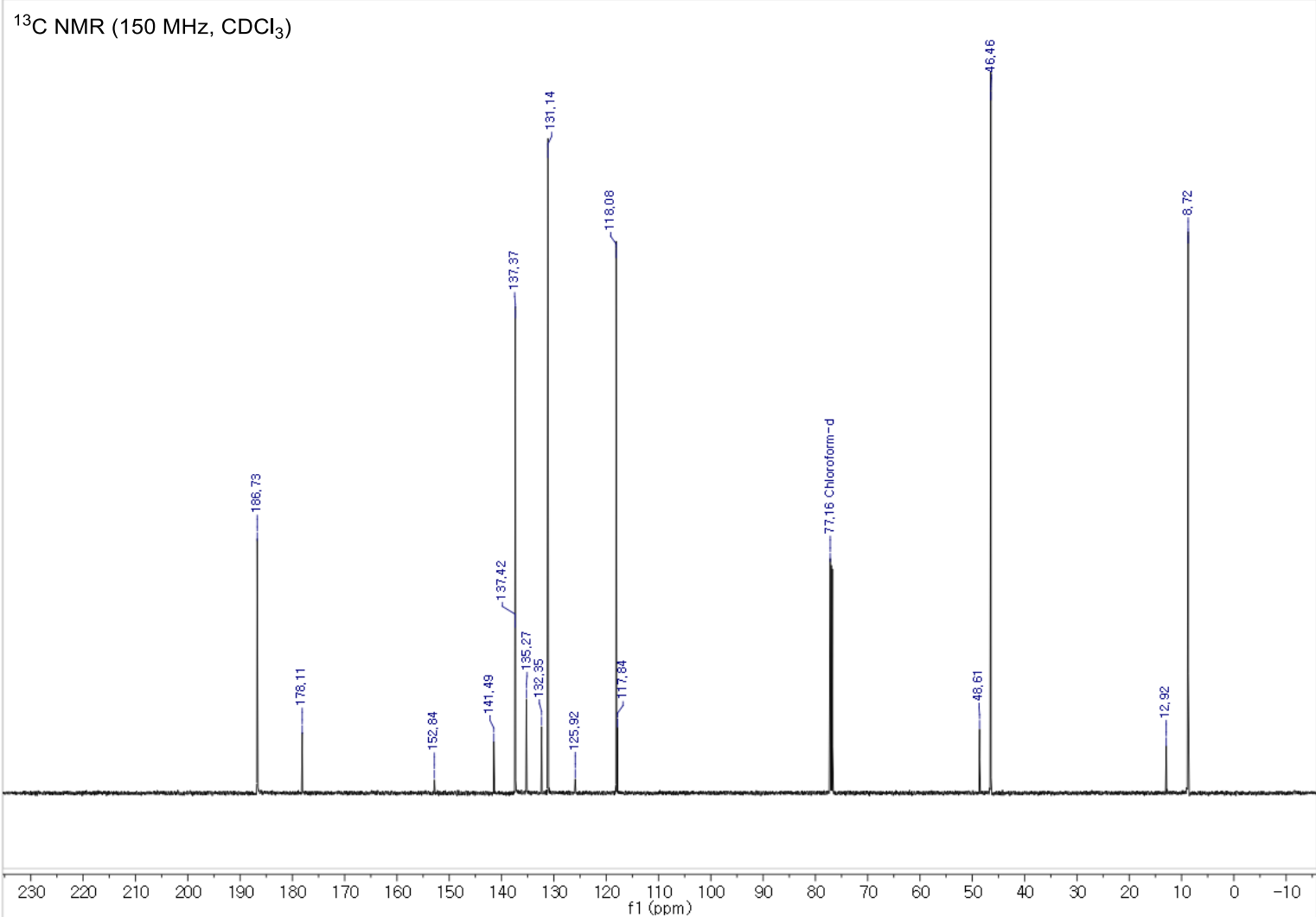


${ }^{1} \mathrm{H} \mathrm{NMR}\left(600 \mathrm{MHz}, \mathrm{CDCl}_{3}\right)$
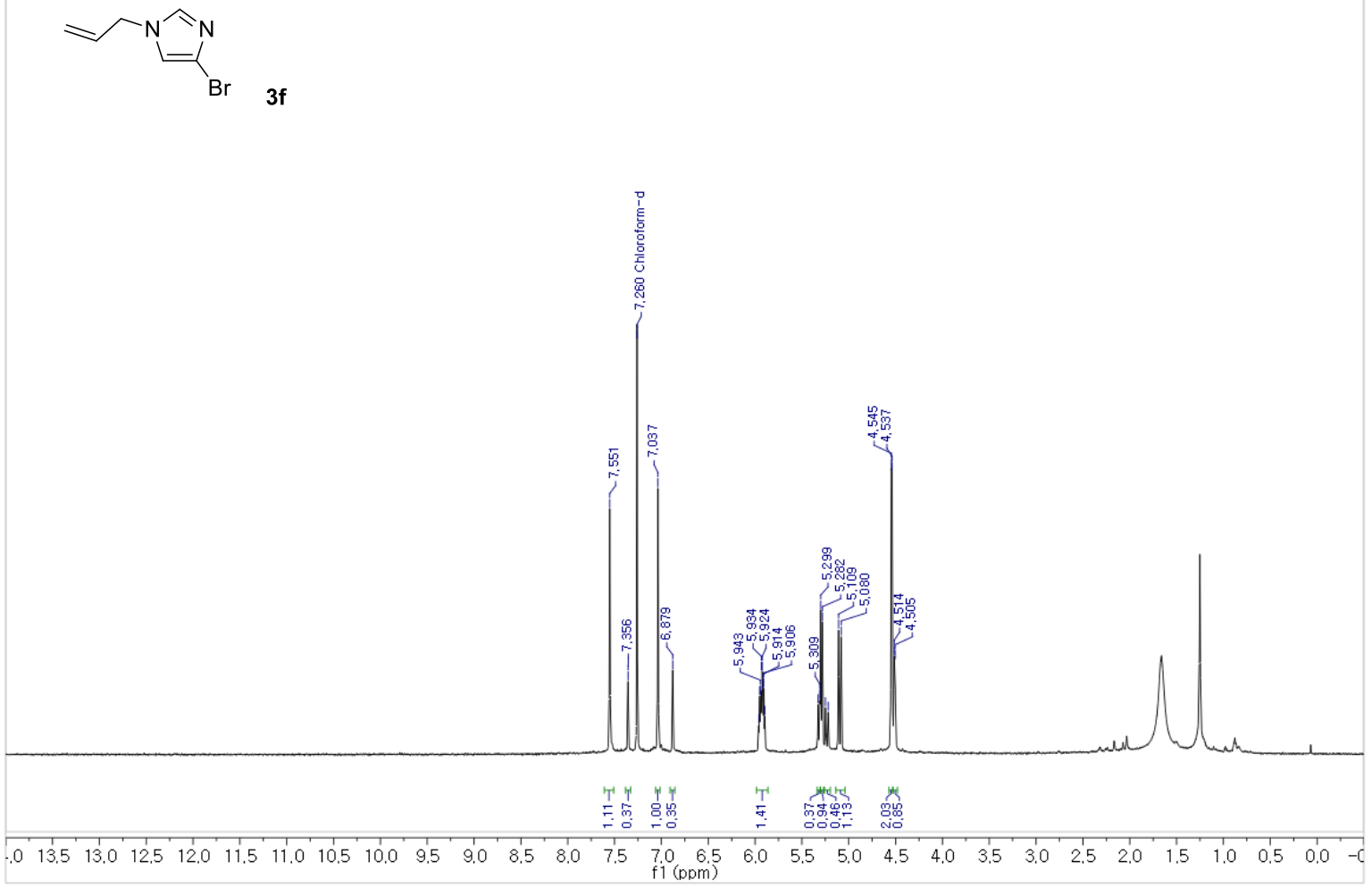

${ }^{13} \mathrm{C}$ NMR (150 MHz, $\mathrm{CDCl}_{3}$ )

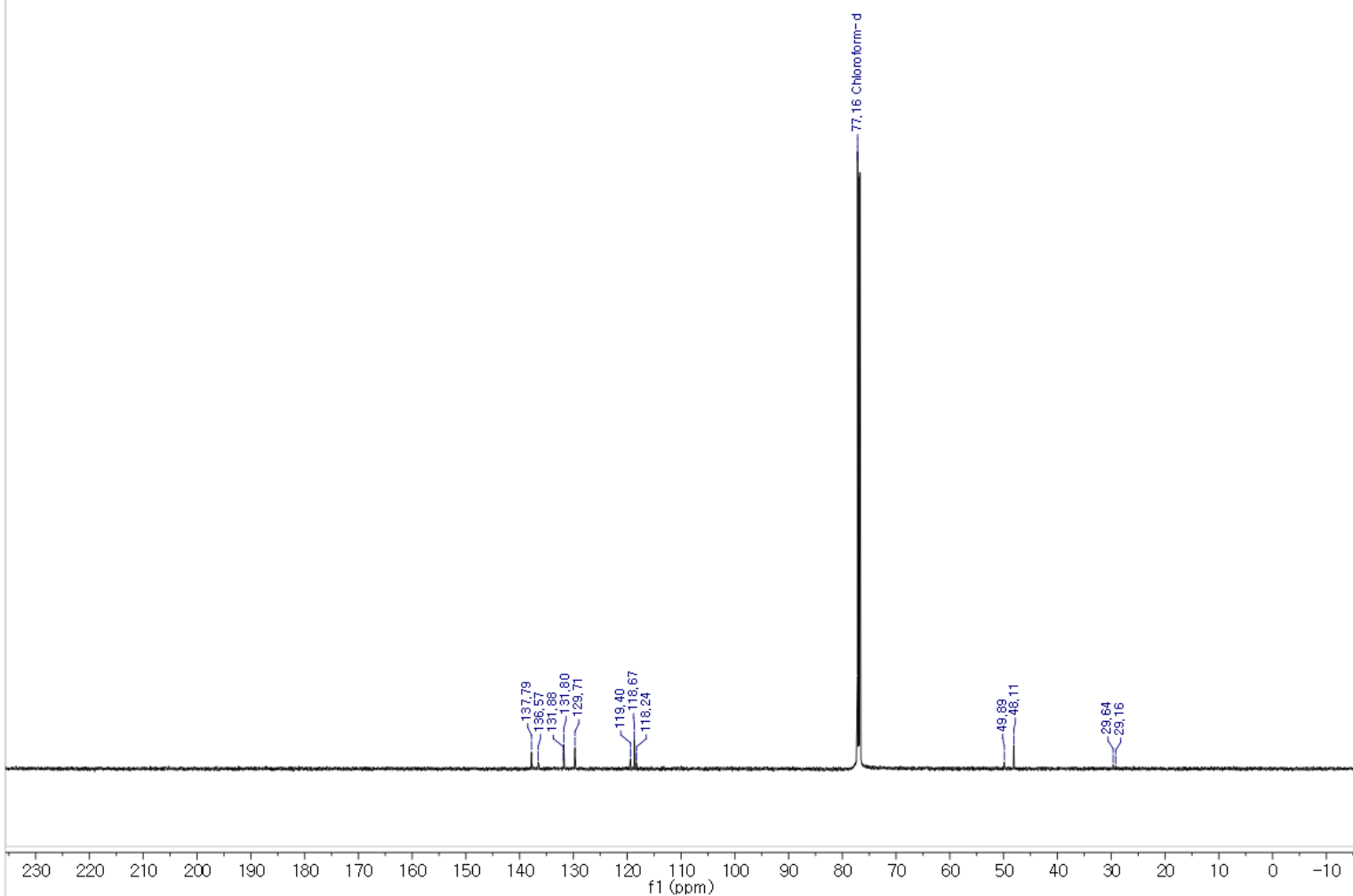


${ }^{1} \mathrm{H}$ NMR $\left(600 \mathrm{MHz}, \mathrm{CDCl}_{3}\right)$

$\approx \sum_{3 g}^{N} \overbrace{}^{N}$

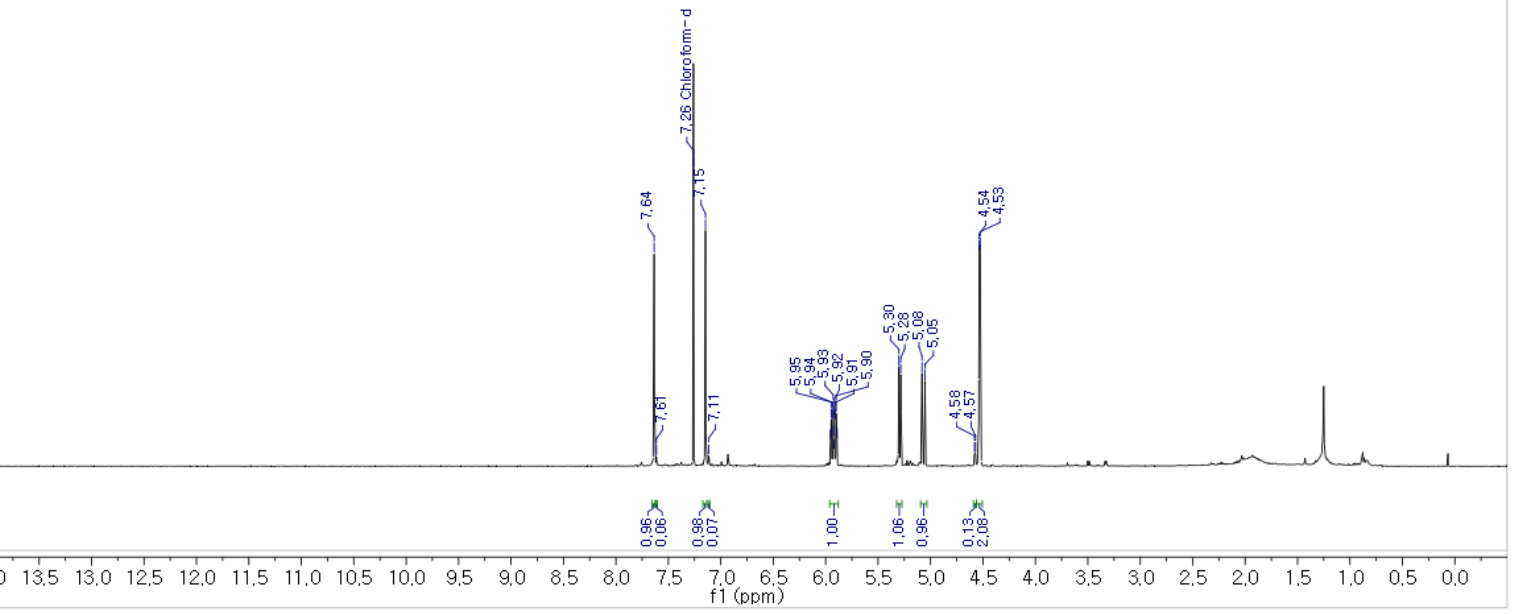

${ }^{13} \mathrm{C}$ NMR (150 MHz, $\mathrm{CDCl}_{3}$ )

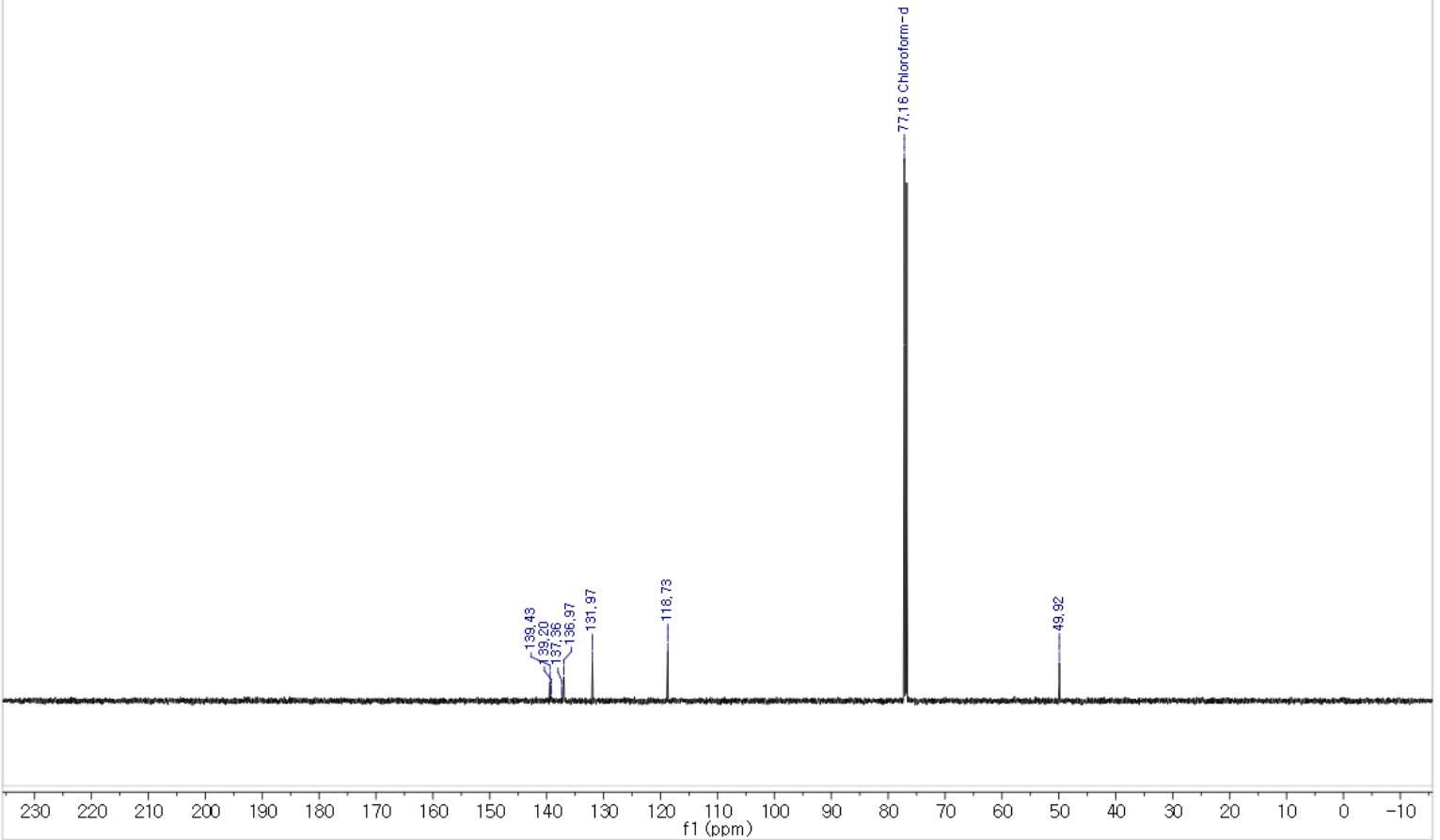


${ }^{1} \mathrm{H}$ NMR $\left(600 \mathrm{MHz}, \mathrm{CDCl}_{3}\right)$<smiles>C=CCn1ccnc1CC</smiles>

$3 h$

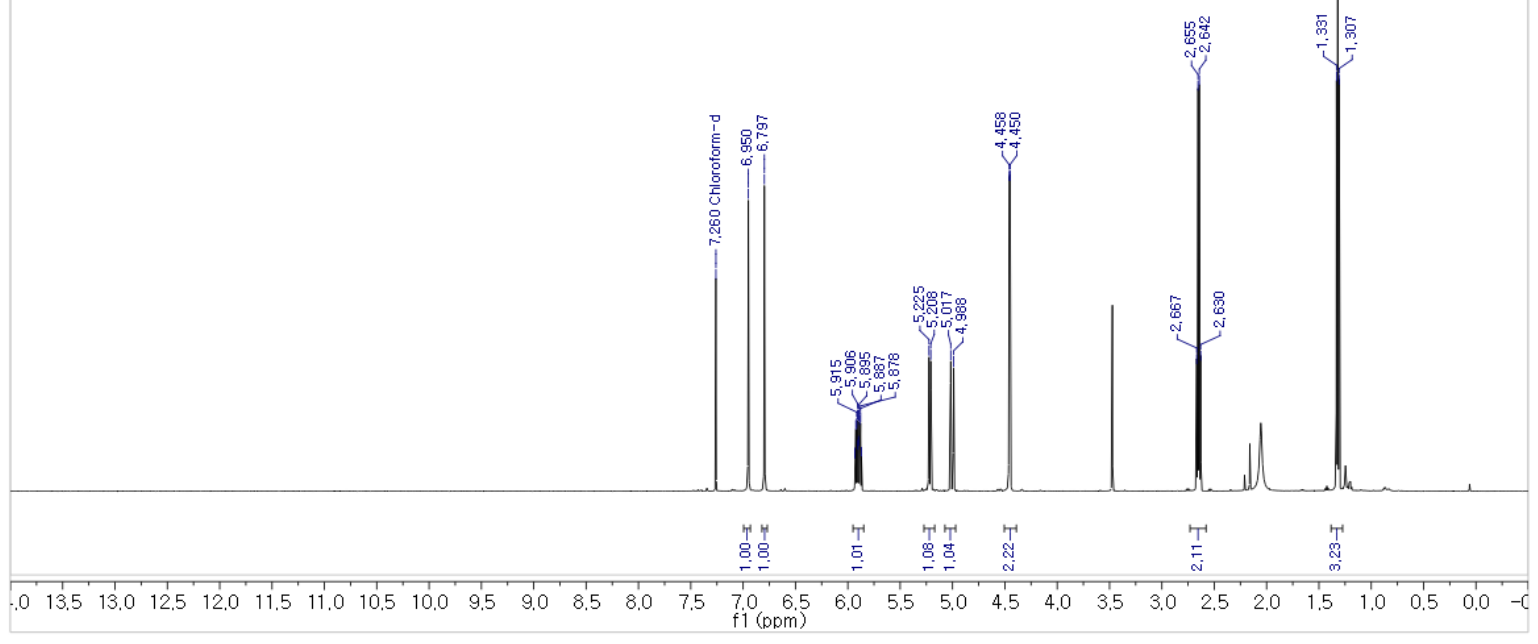

${ }^{13} \mathrm{C}$ NMR (150 MHz, $\mathrm{CDCl}_{3}$ )

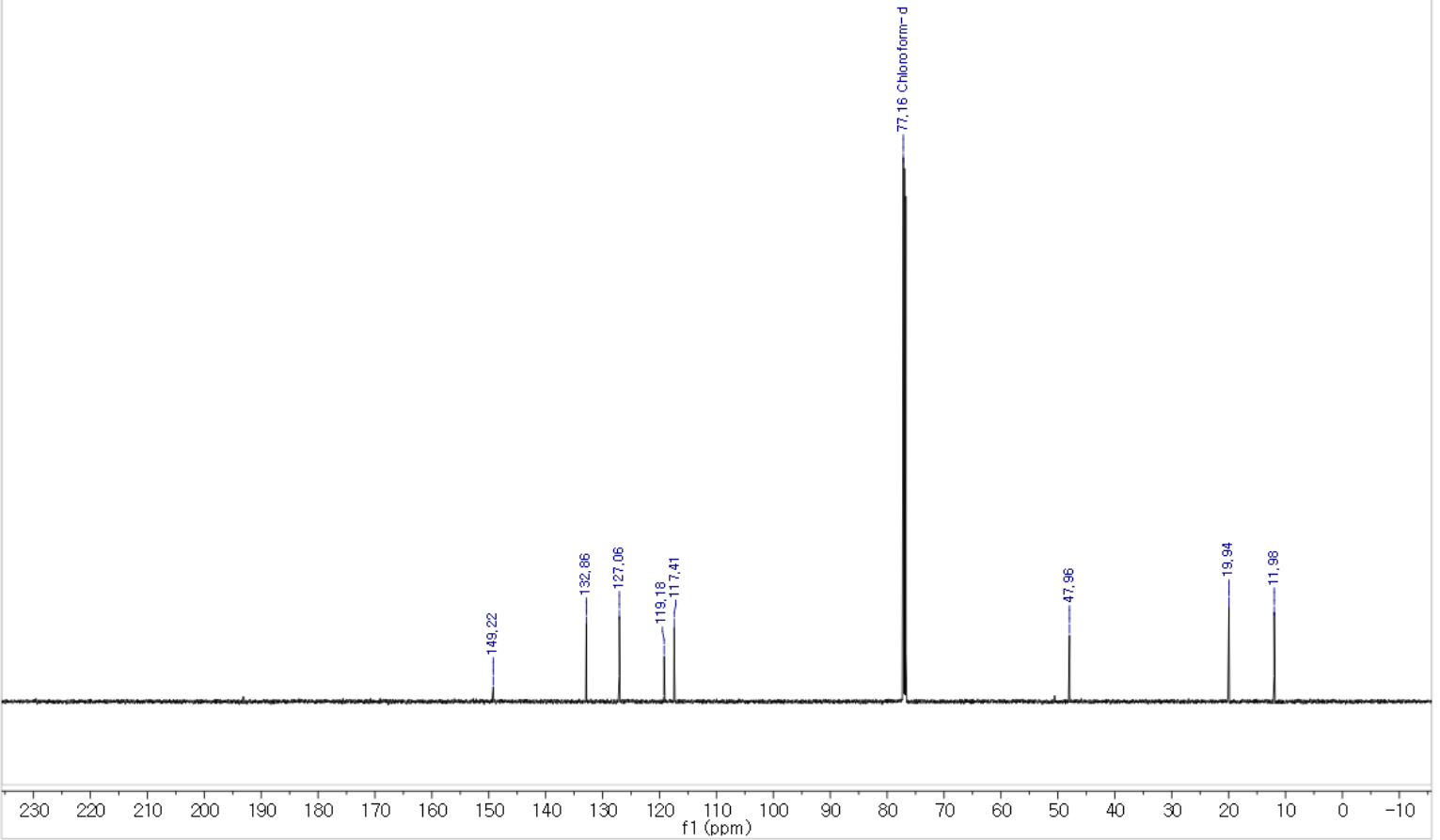



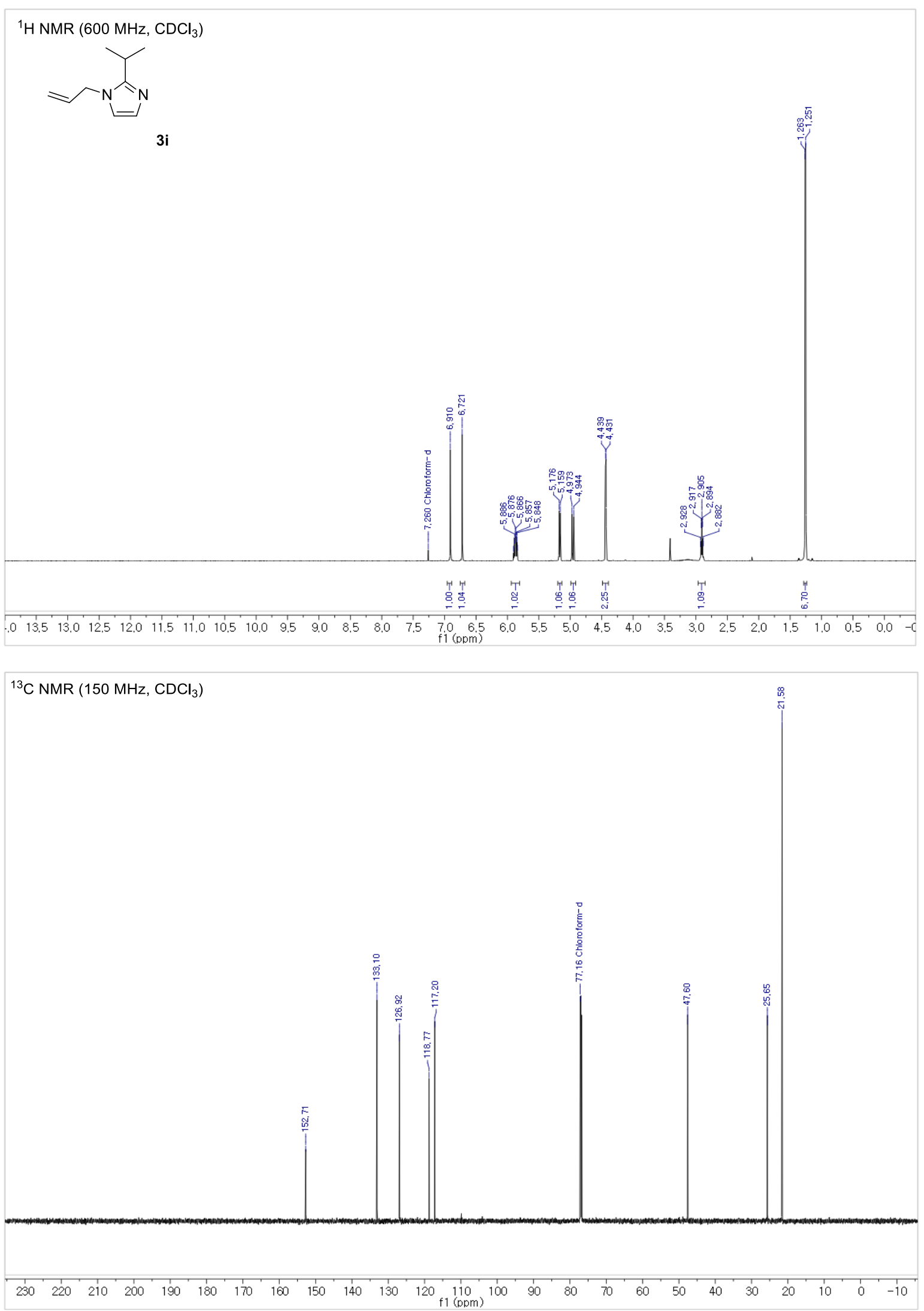


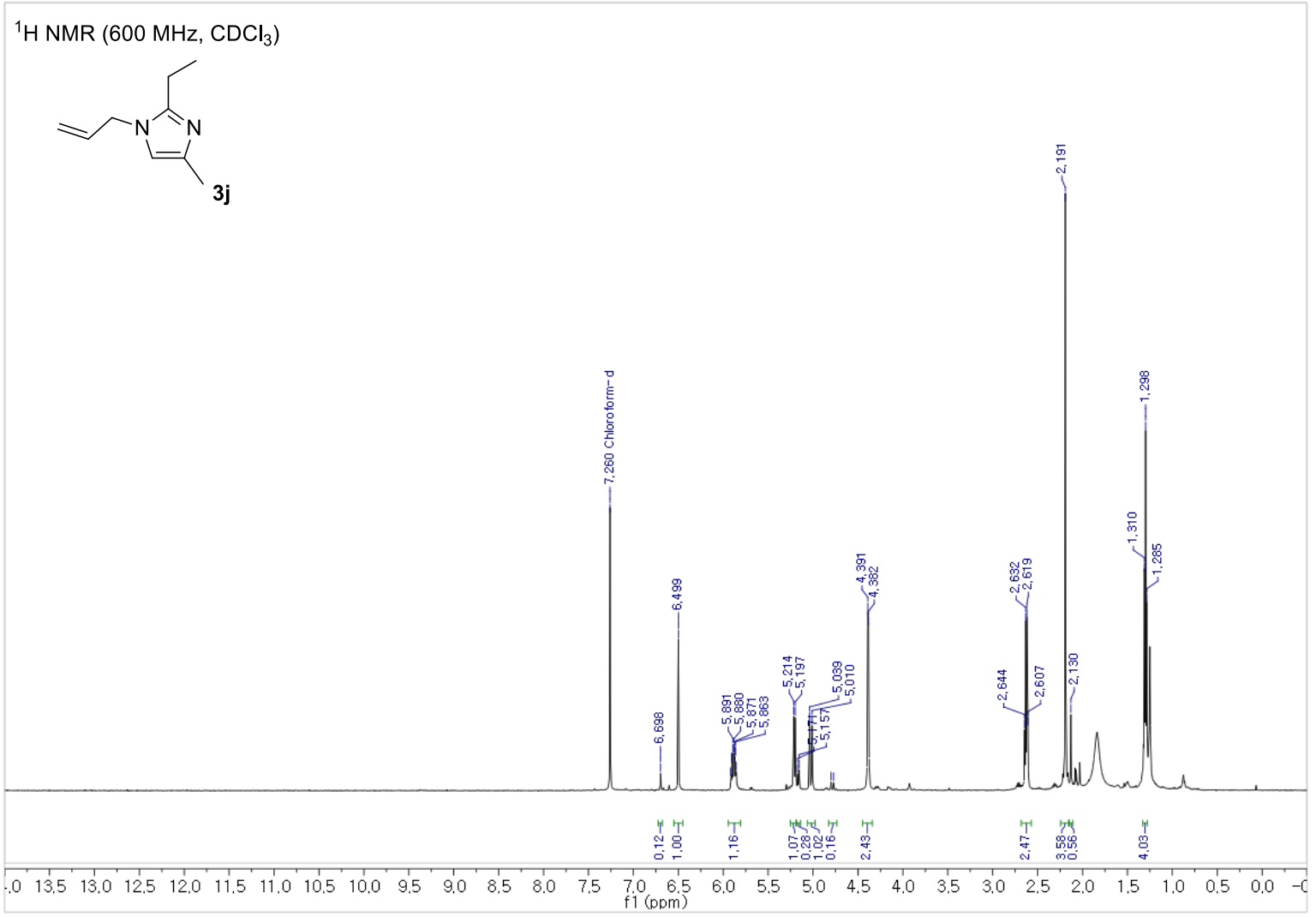

${ }^{13} \mathrm{C}$ NMR (150 MHz, $\mathrm{CDCl}_{3}$ )

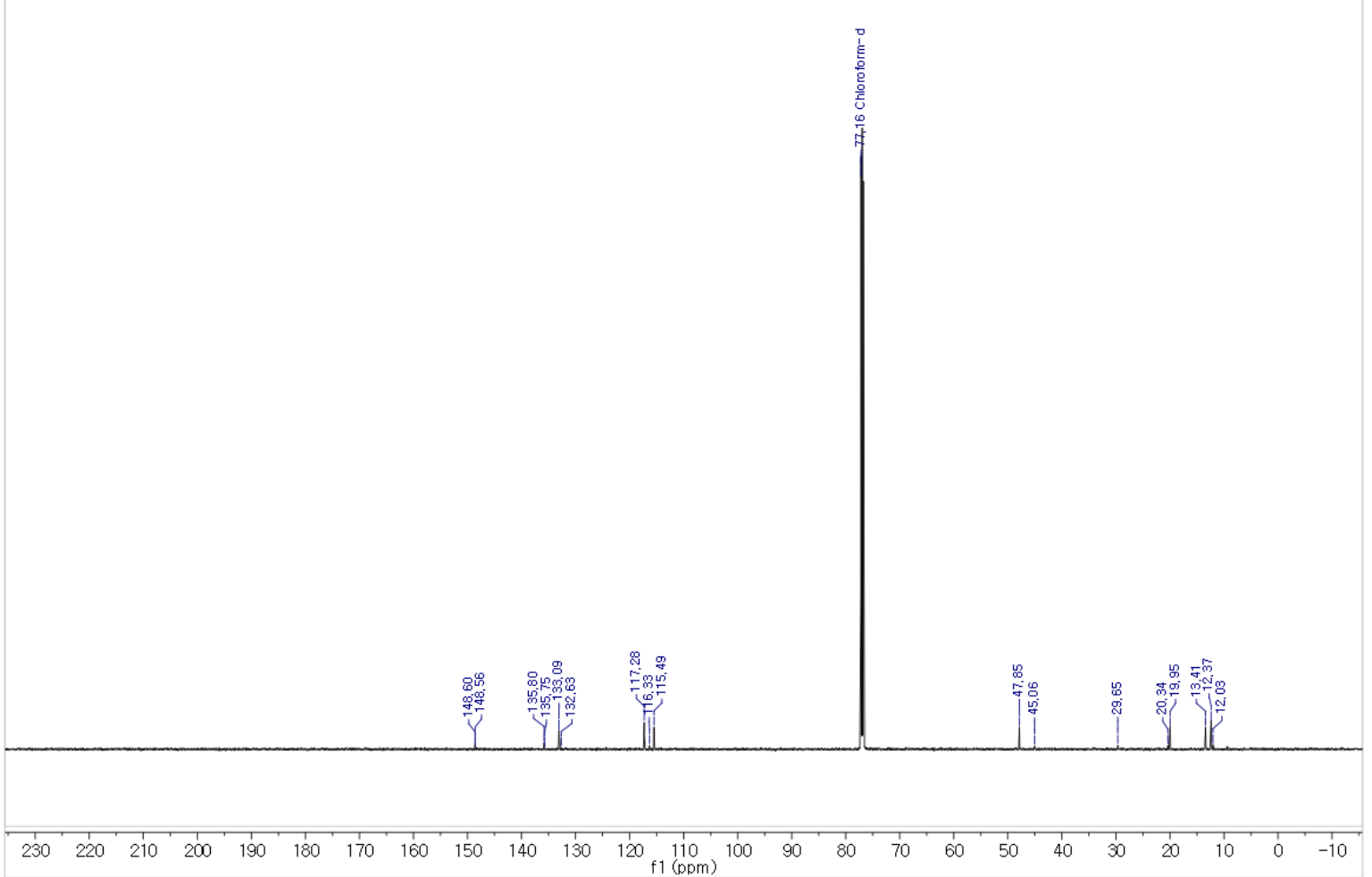




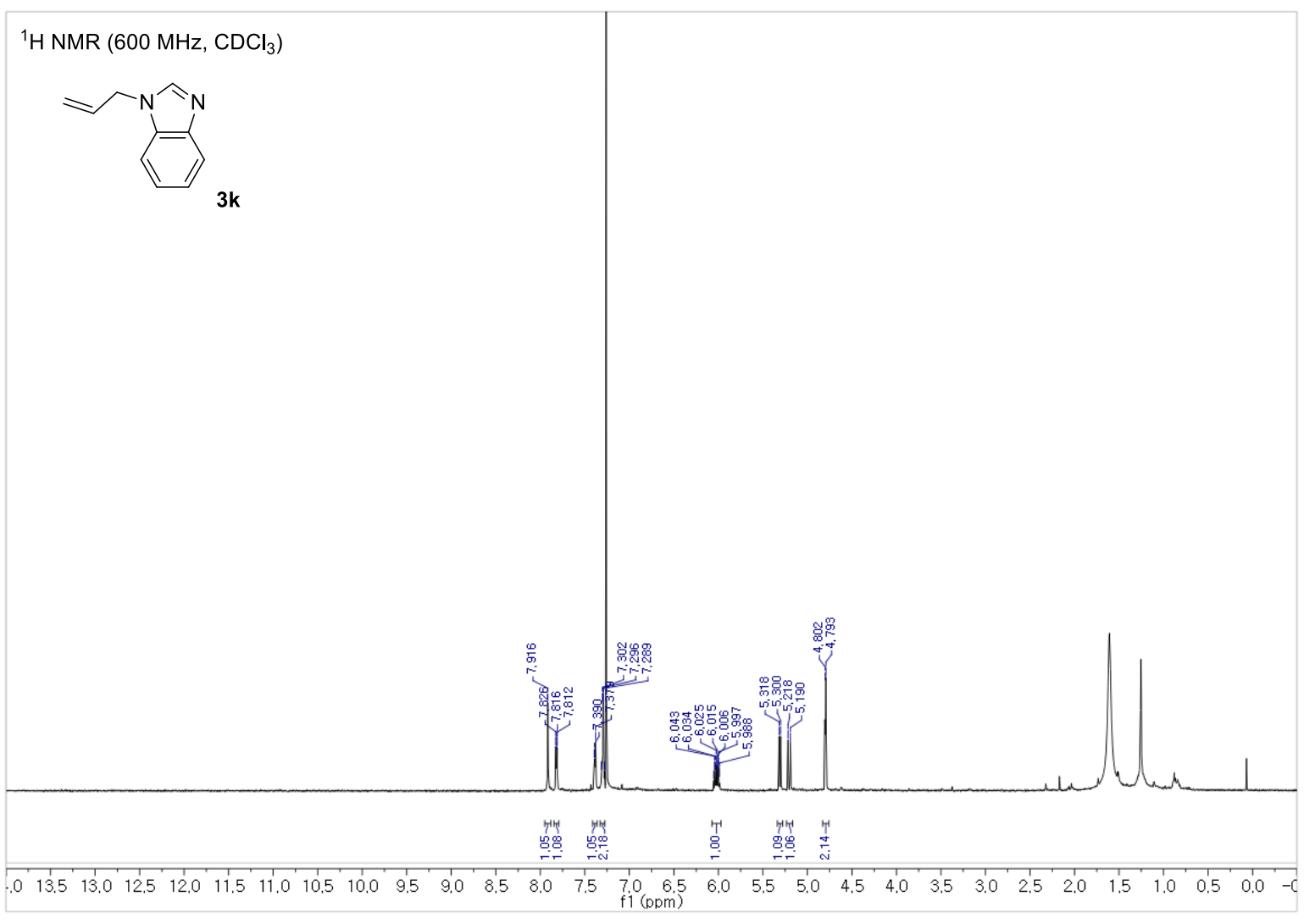

${ }^{13} \mathrm{C}$ NMR (150 MHz, $\mathrm{CDCl}_{3}$ )

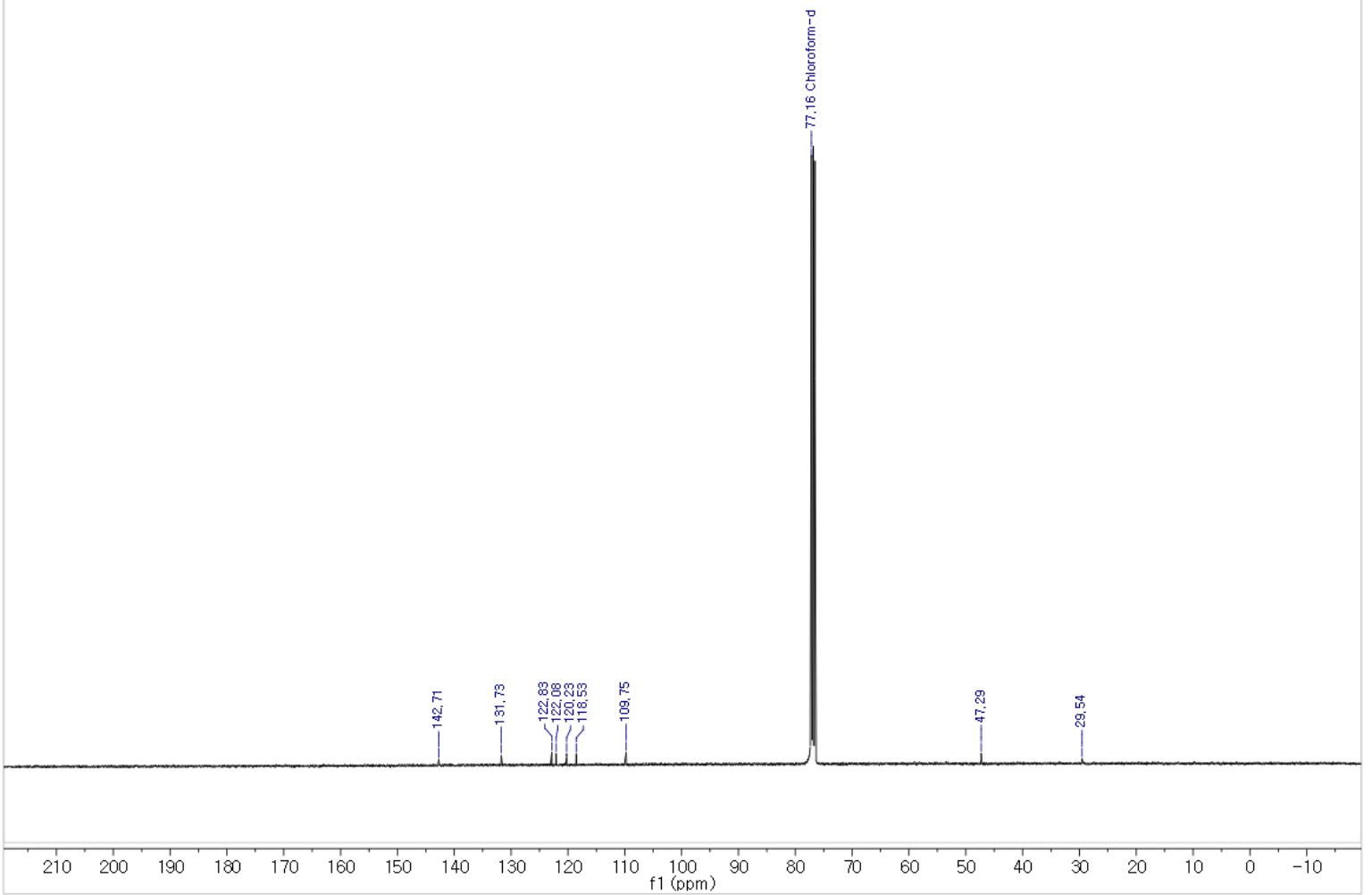


${ }^{1} \mathrm{H}$ NMR (600 MHz, $\mathrm{CDCl}_{3}$ )

$\approx{ }_{31}^{N}$

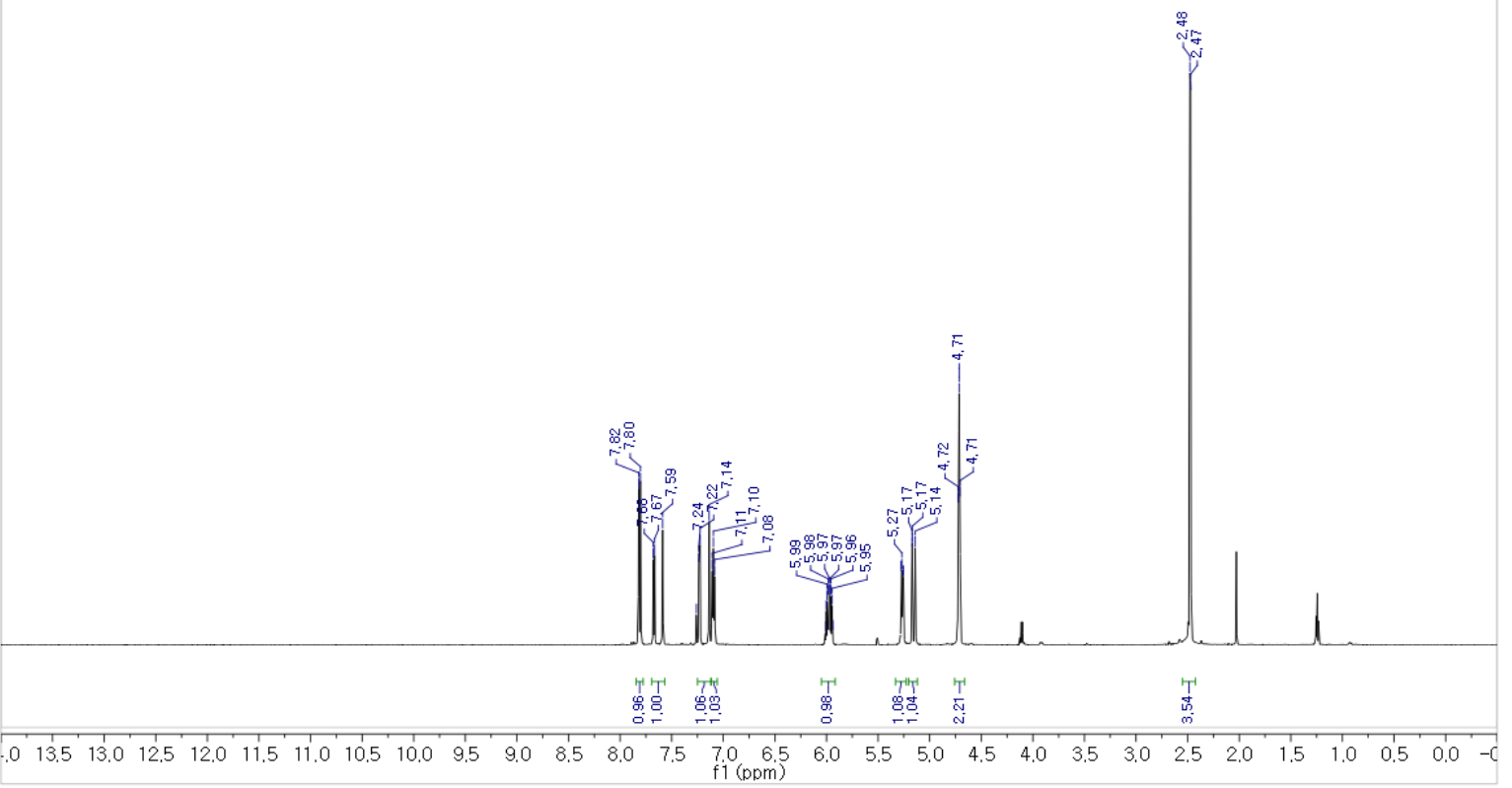

$\left.{ }^{13} \mathrm{C} \mathrm{NMR} \mathrm{(150} \mathrm{MHz,} \mathrm{CDCl}_{3}\right)$

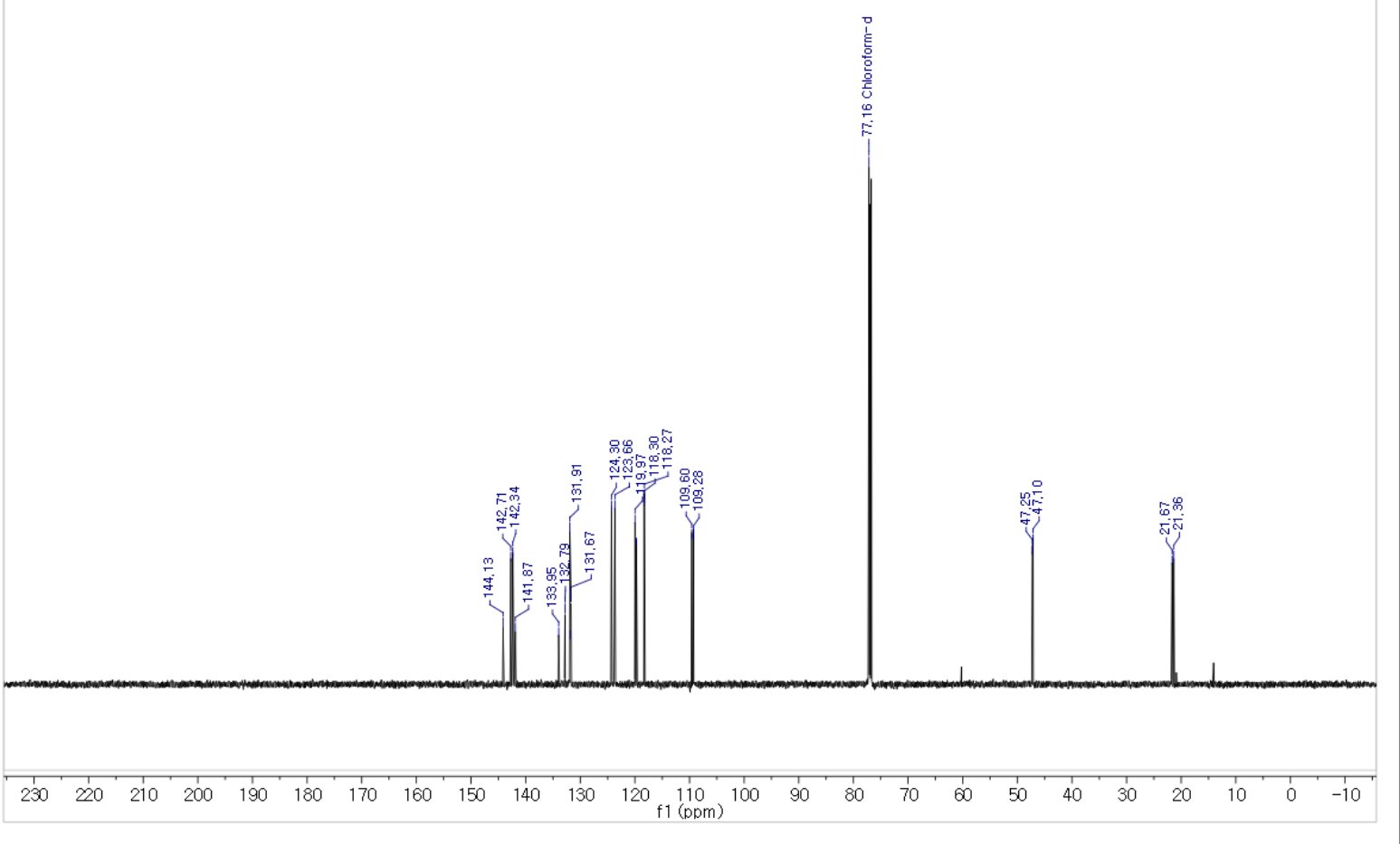




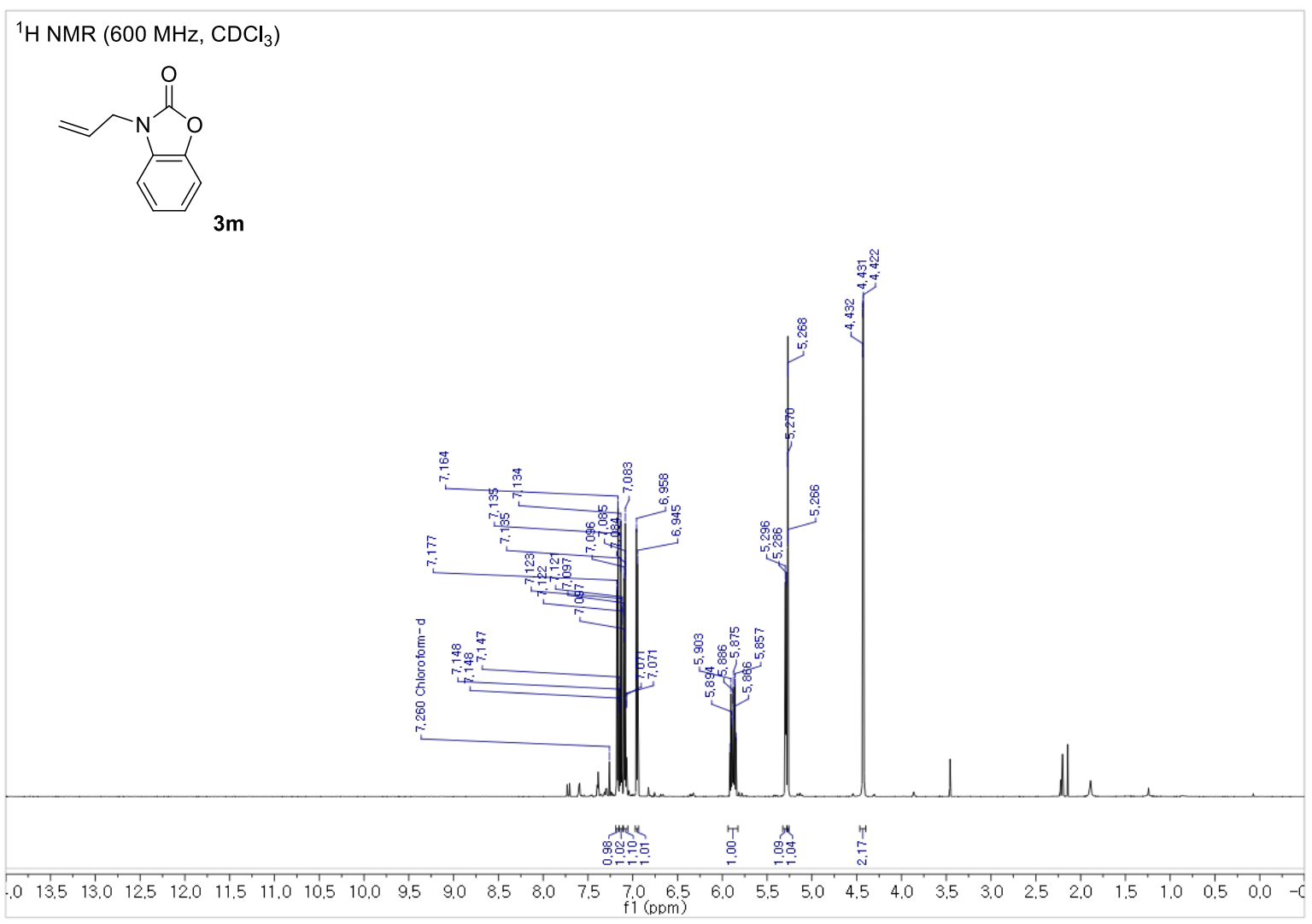

${ }^{13} \mathrm{C}$ NMR (150 MHz, $\mathrm{CDCl}_{3}$ )

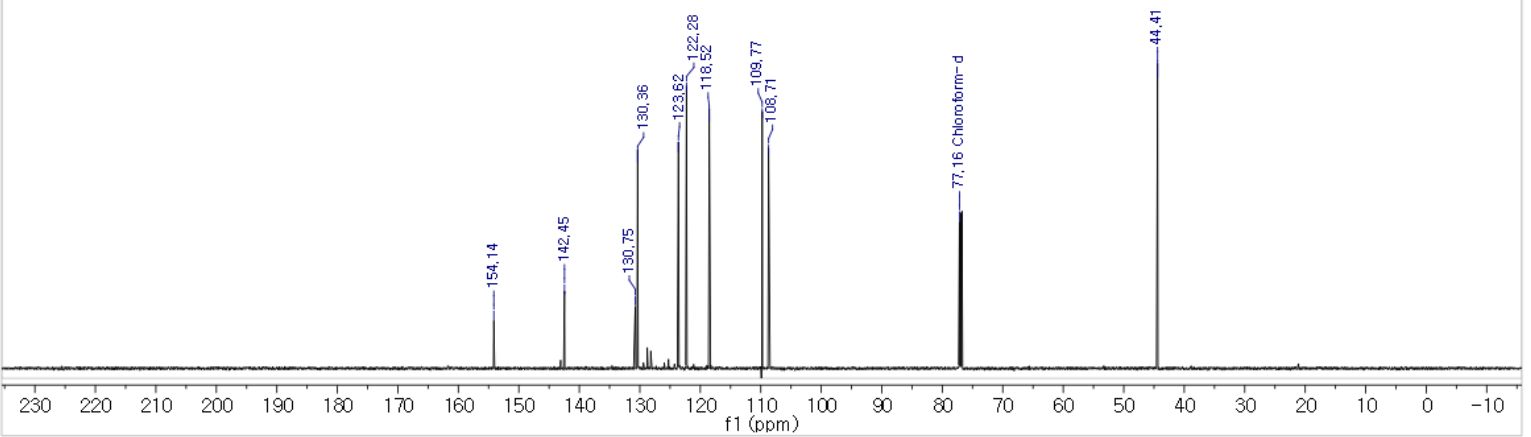


${ }^{1} \mathrm{H} \mathrm{NMR}\left(600 \mathrm{MHz}, \mathrm{CDCl}_{3}\right)$

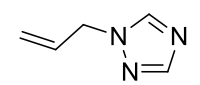

3n

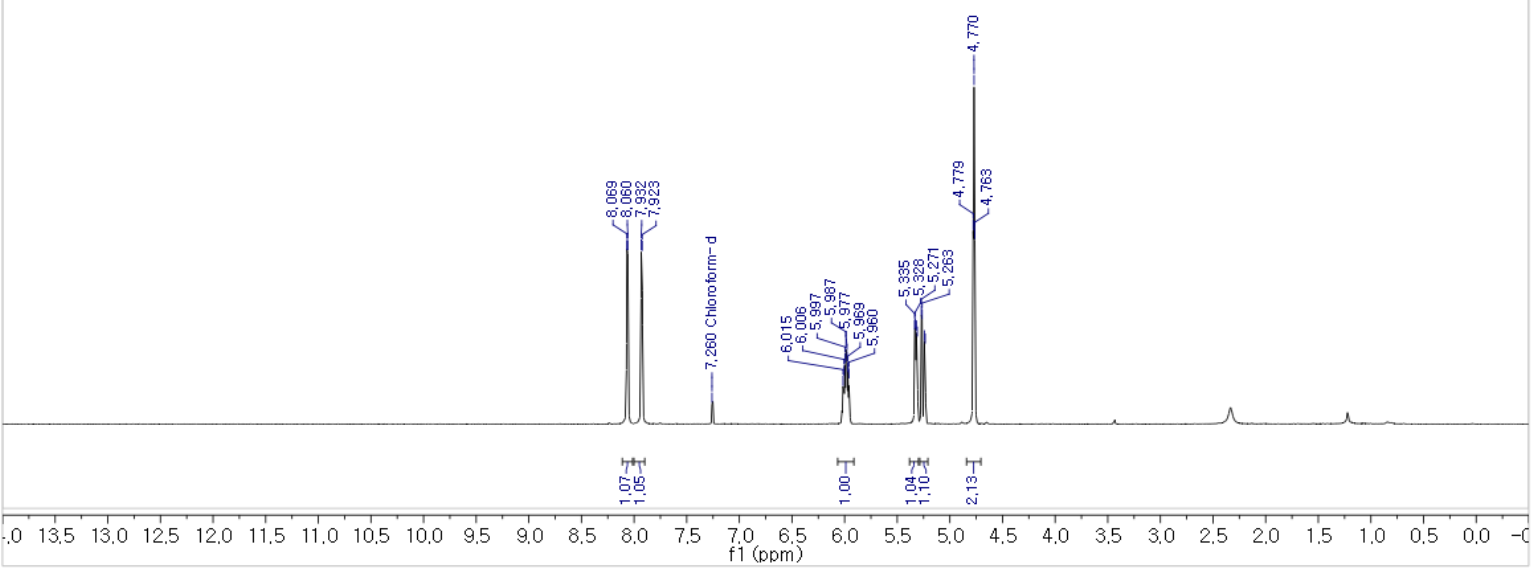

${ }^{13} \mathrm{C} \mathrm{NMR} \mathrm{(150} \mathrm{MHz,} \mathrm{CDCl}_{3}$ )

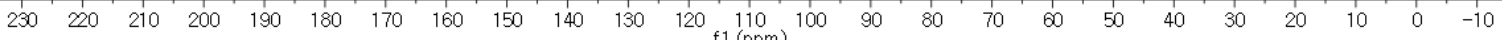


${ }^{1} \mathrm{H} \mathrm{NMR}\left(600 \mathrm{MHz}, \mathrm{CDCl}_{3}\right)$

$\approx \underset{\mathrm{N}=}{\mathrm{N}}$

30

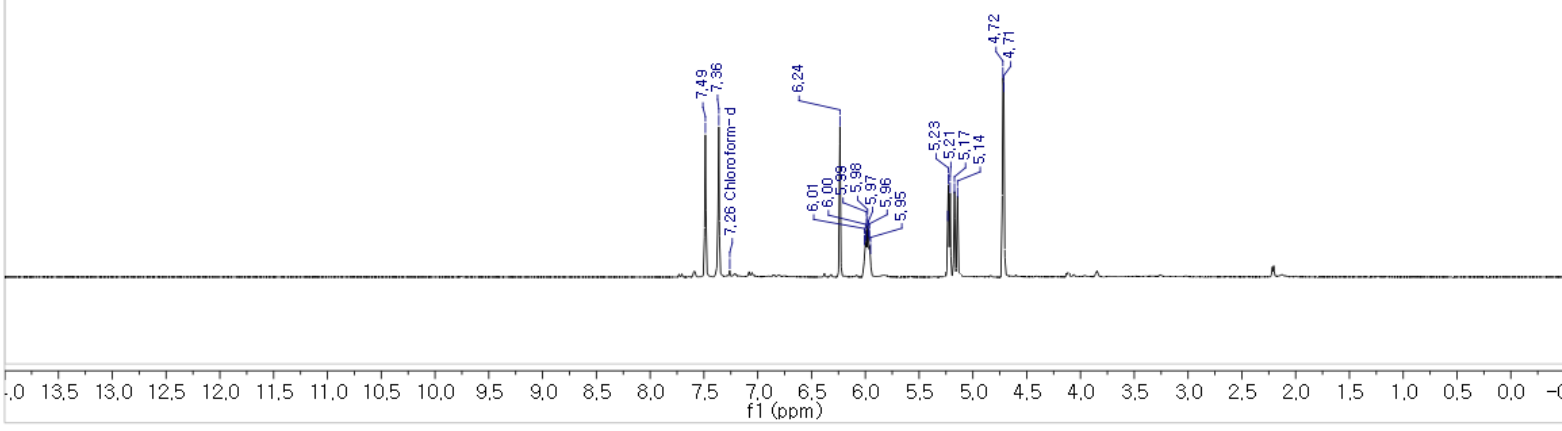

$\left.{ }^{13} \mathrm{C} \mathrm{NMR} \mathrm{(150} \mathrm{MHz,} \mathrm{CDCl}_{3}\right)$

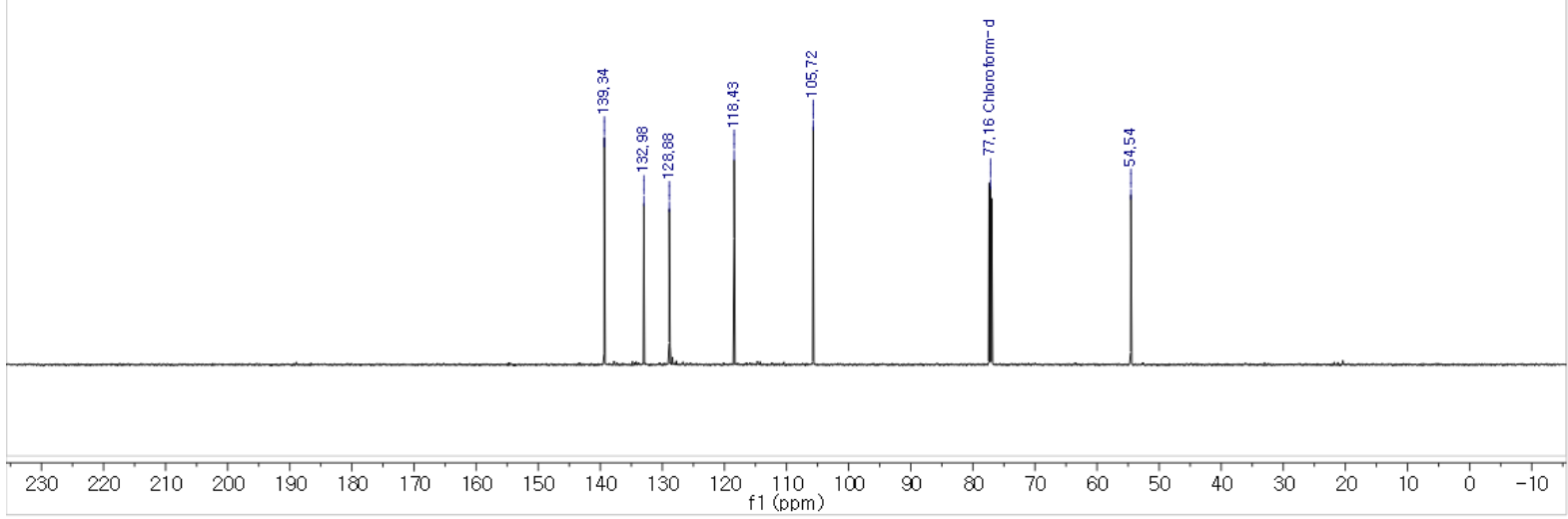




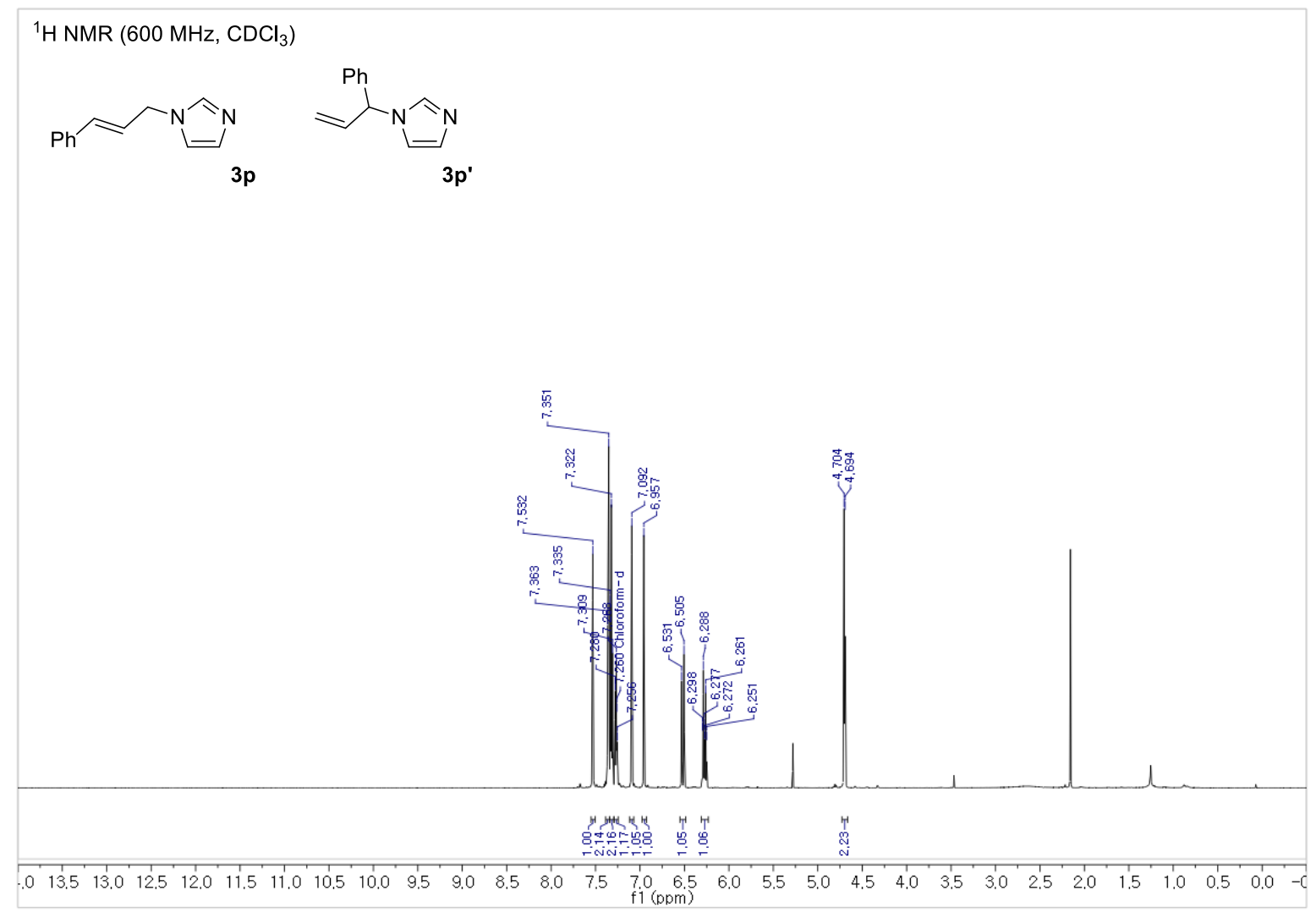

${ }^{13} \mathrm{CNMR}\left(150 \mathrm{MHz}, \mathrm{CDCl}_{3}\right)$

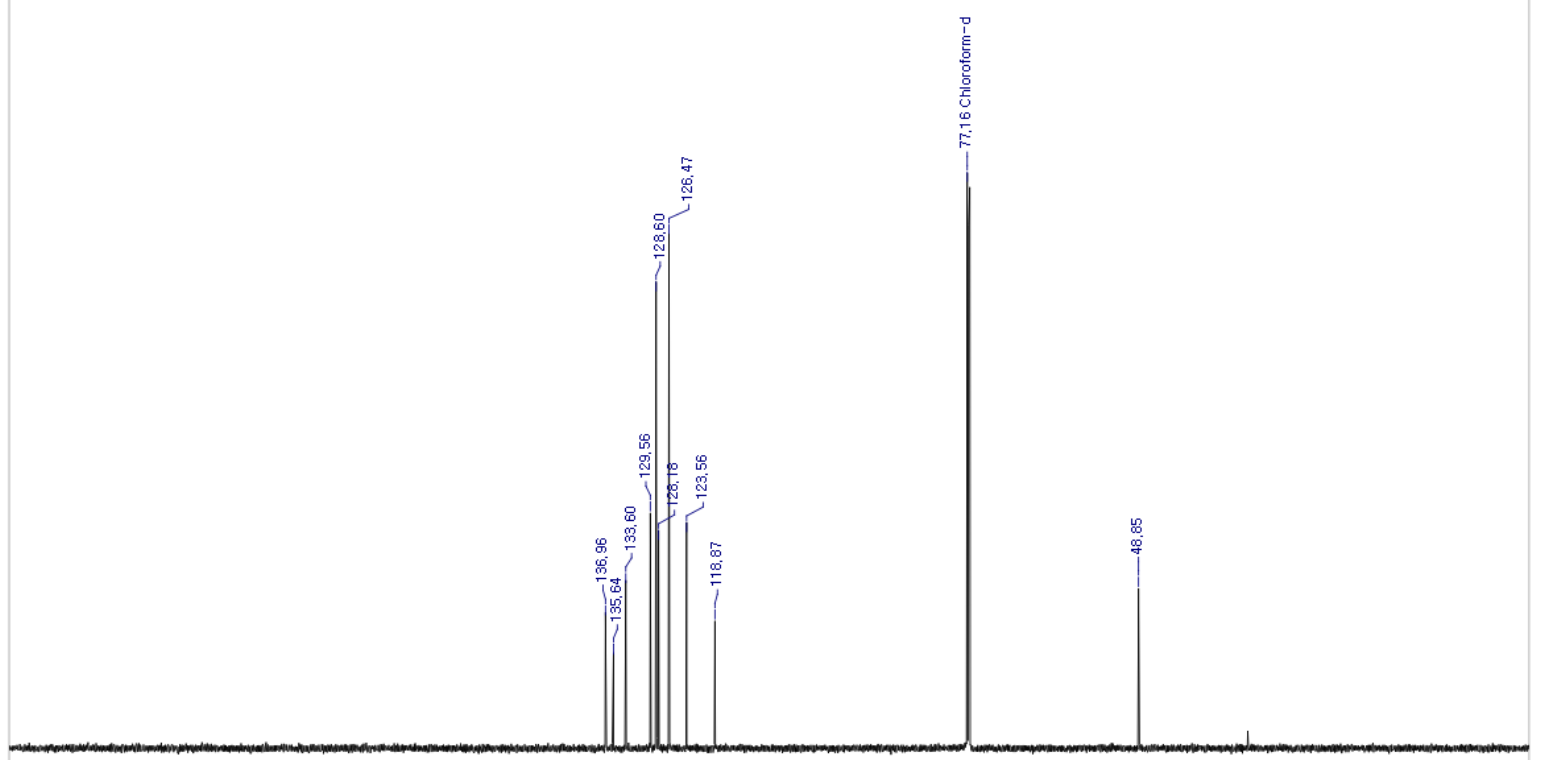

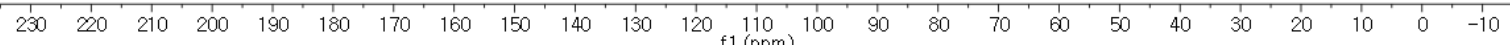


${ }^{1} \mathrm{H}$ NMR $\left(600 \mathrm{MHz}, \mathrm{CDCl}_{3}\right)$

$$
\lambda \underbrace{N_{n}}_{3 q} \Rightarrow \underbrace{}_{3 q^{\prime}}
$$

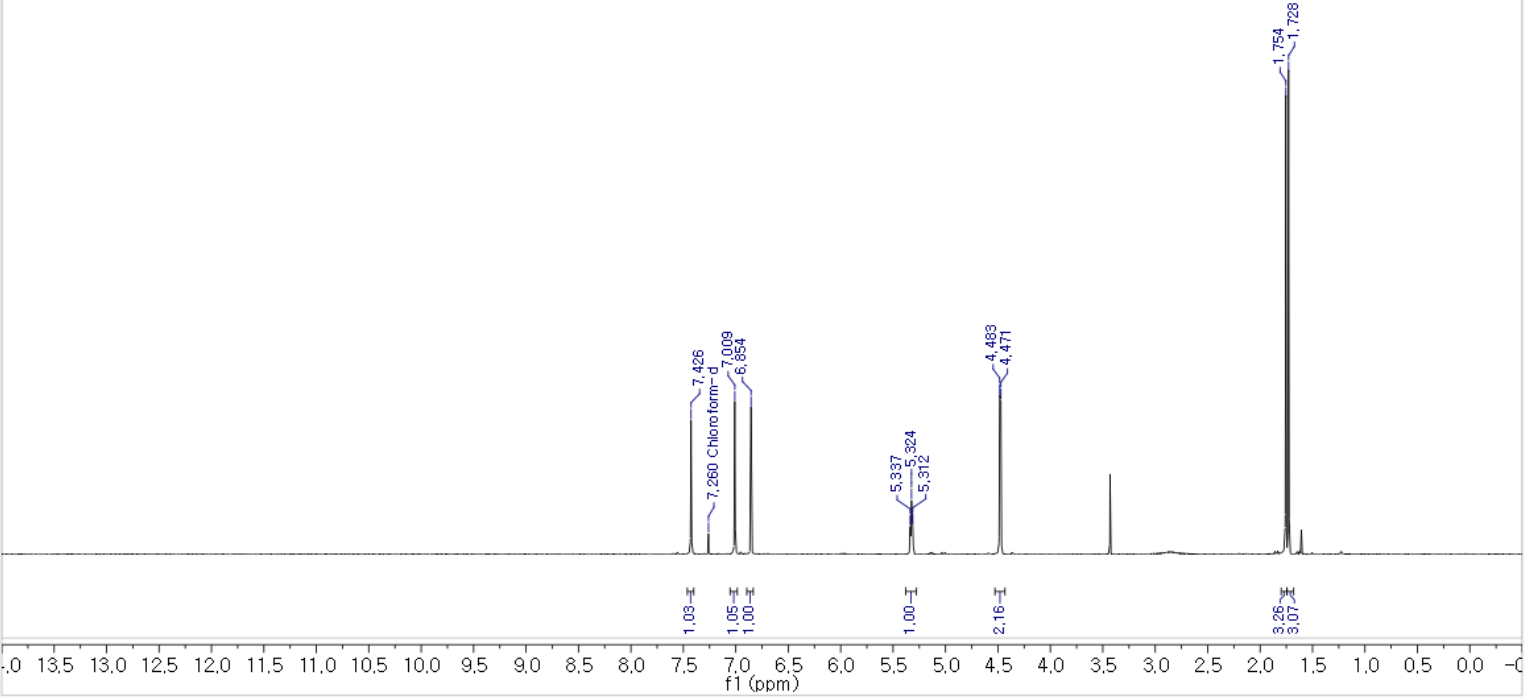

${ }^{13} \mathrm{C}$ NMR $\left(150 \mathrm{MHz}, \mathrm{CDCl}_{3}\right)$

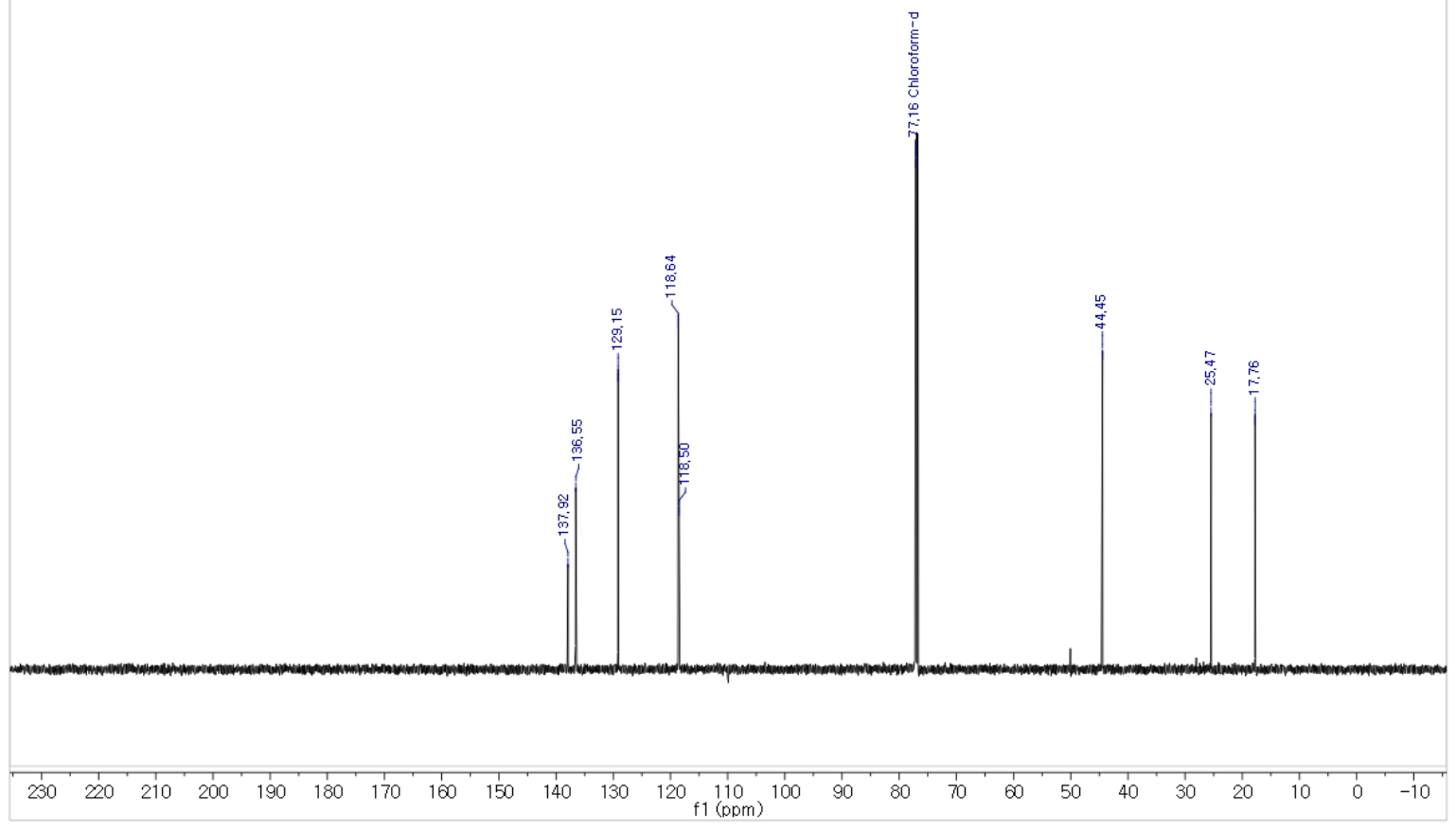


${ }^{1} \mathrm{H} \mathrm{NMR}\left(600 \mathrm{MHz}, \mathrm{CDCl}_{3}\right)$

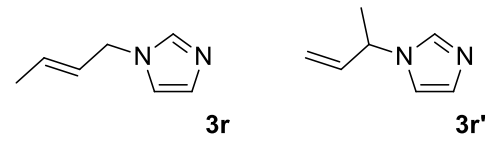

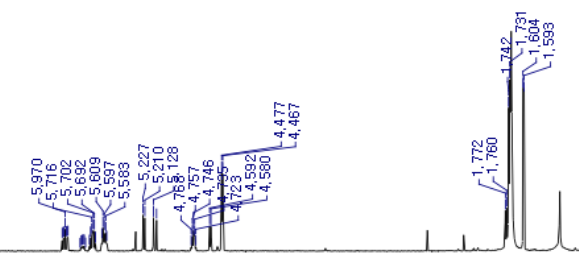

${ }^{13} \mathrm{C} \mathrm{NMR}\left(150 \mathrm{MHz}, \mathrm{CDCl}_{3}\right)$

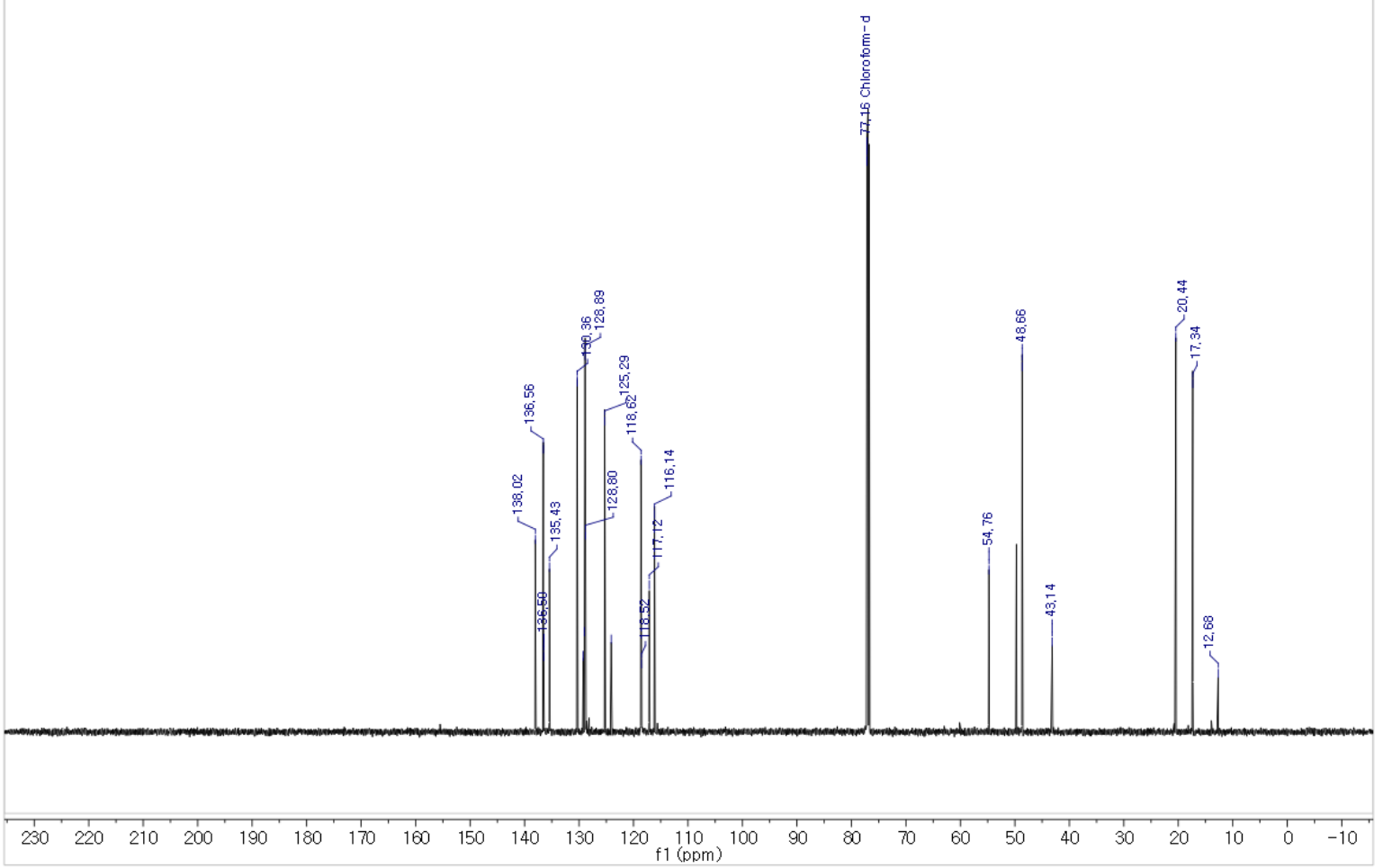


${ }^{1} \mathrm{H} \mathrm{NMR}\left(600 \mathrm{MHz}, \mathrm{CDCl}_{3}\right)$

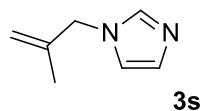

3s

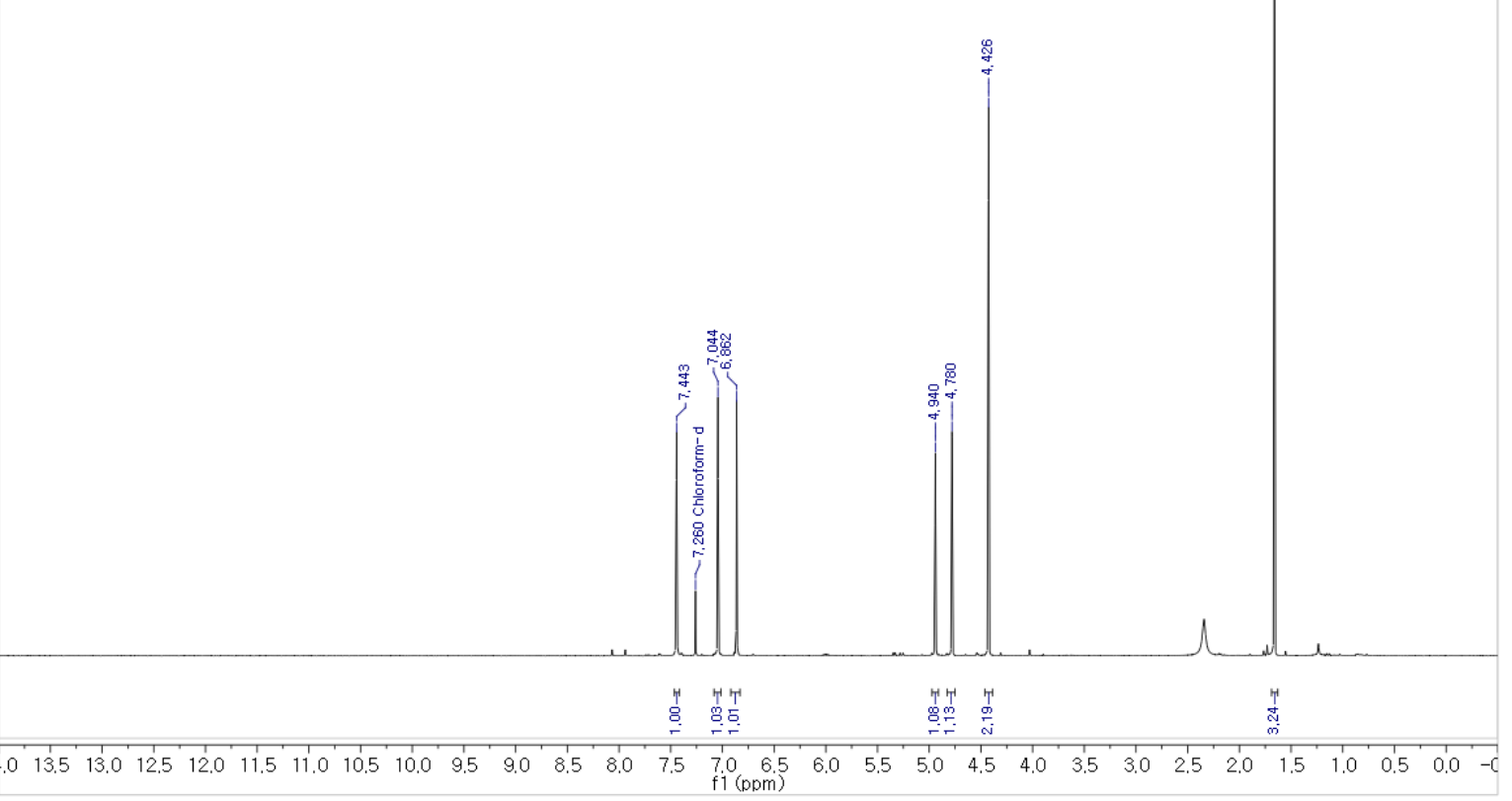

${ }^{13} \mathrm{C} \mathrm{NMR}\left(150 \mathrm{MHz}, \mathrm{CDCl}_{3}\right)$

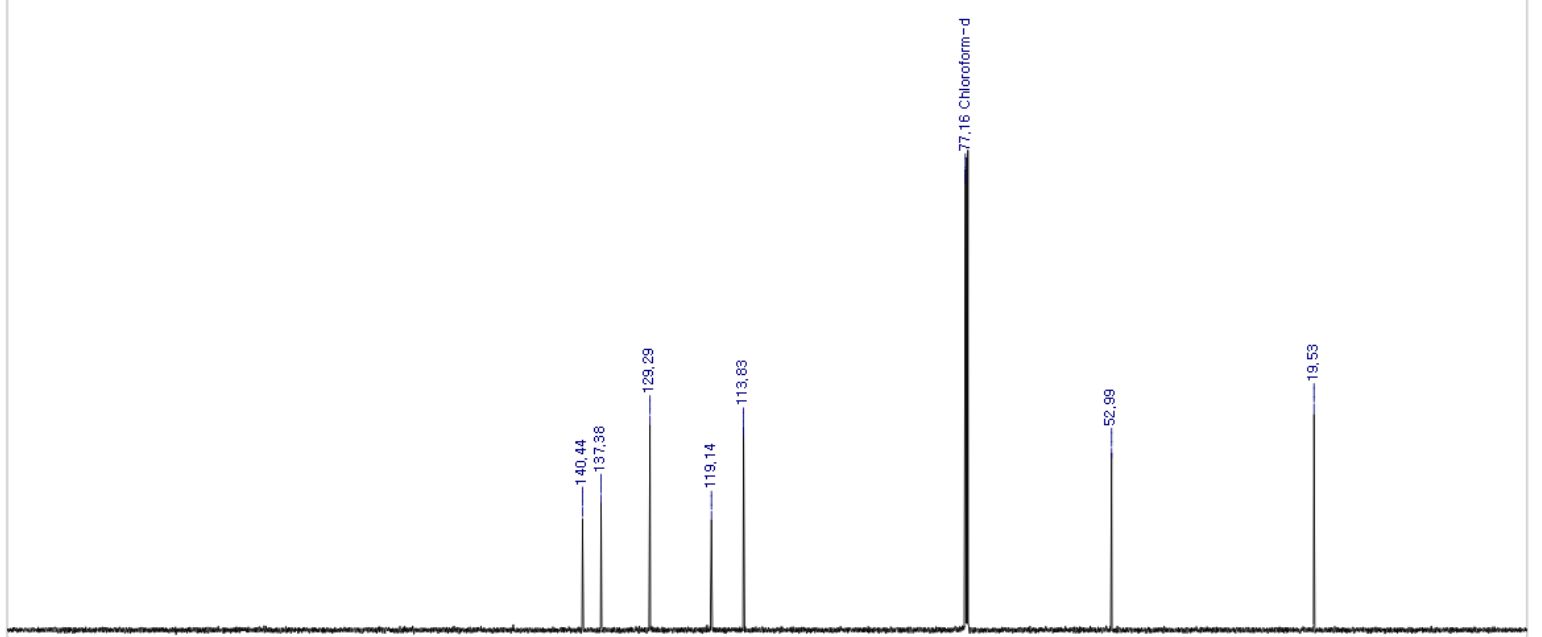

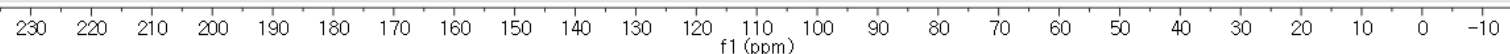




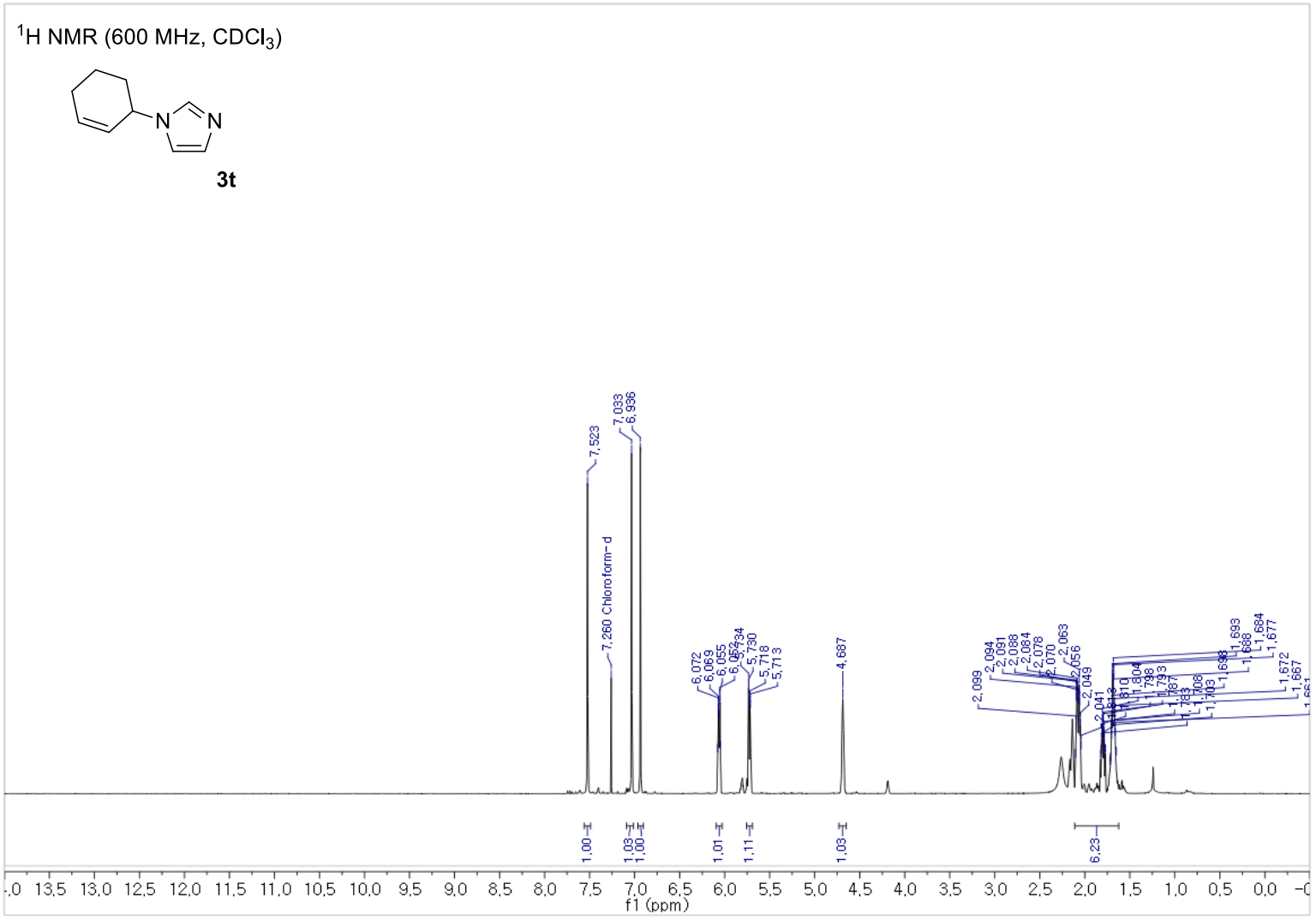

${ }^{13} \mathrm{C}$ NMR (150 MHz, $\mathrm{CDCl}_{3}$ )

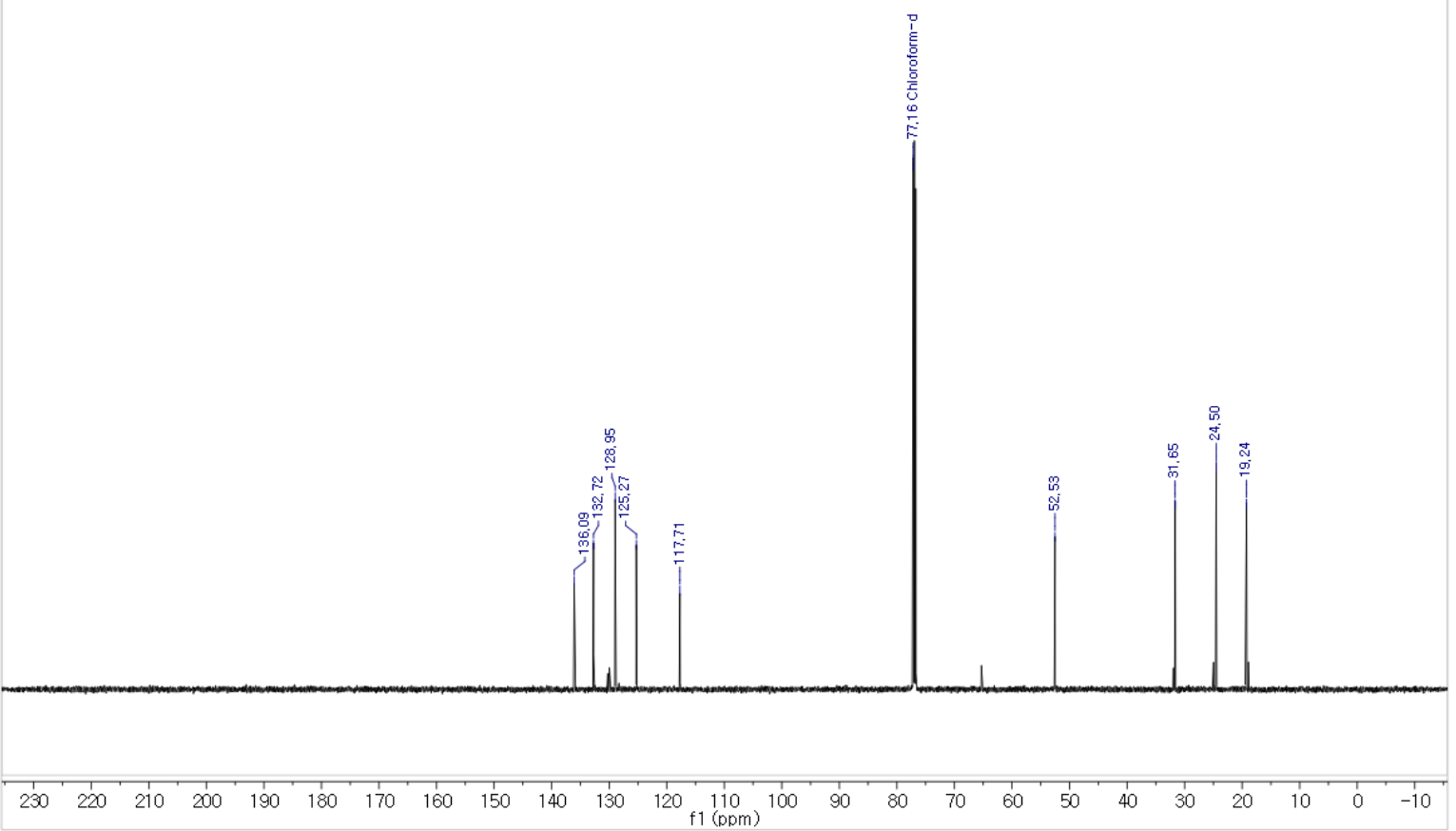



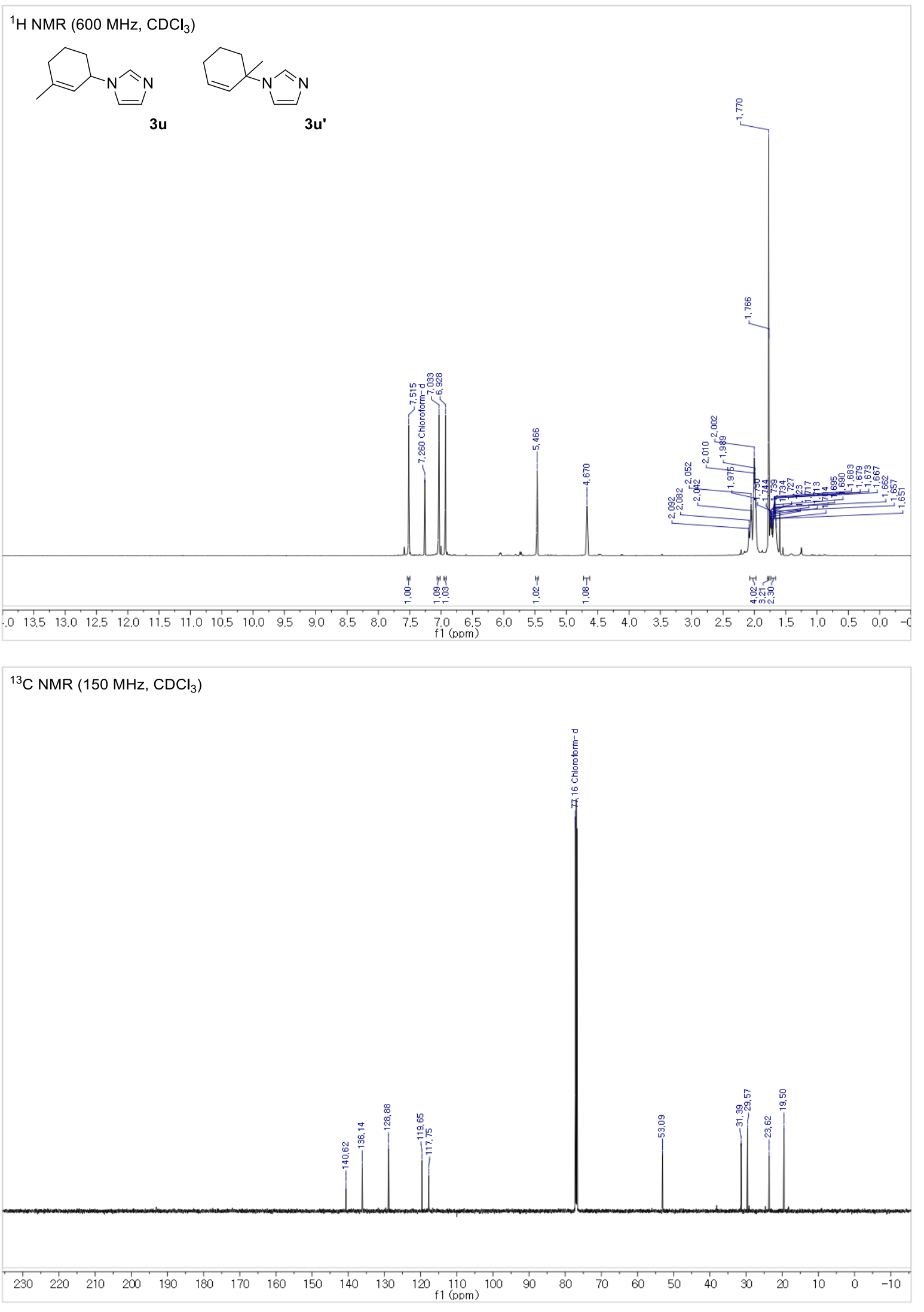